\title{
Chemical Equilibrium of Zinc-Acetate Complexes in Ethanol Solution. A Theoretical Description Through Thermodynamic Cycles
}

Julieta Reyna-Luna ${ }^{1,2}$, Raúl Flores ${ }^{2}$, Rodolfo Gómez-Balderas ${ }^{2 *}$, Marco Franco-Pérez $^{1}{ }^{*}$

1) Departamento de Física y Química Teórica, Facultad de Química, Universidad Nacional Autónoma de México, Cd. Universitaria, 04510 Ciudad de México, México.

2) Laboratorio de Fisicoquímica Analítica, Unidad de Investigación Multidisciplinaria, Facultad de Estudios Superiores-Cuautitlán, Universidad Nacional Autónoma de México, Cuautitlán Izcalli, 54700, Estado de México, México.

\section{Supporting Information}

*Corresponding authors: qimfranco@hotmail.com, rodolfo.gomez@unam.mx 


\section{Index}

Figure S1. Relevant parameters of optimized geometries in the gas phase optimized for linear-gauche ethanol clusters: a) trimer, b) tetramer, c) pentamer, d) hexamer, e) heptamer and f) octamer, at the level M05-2X/6-31+G(d)

Figure S2. Relevant parameters of optimized geometries in the gas phase optimized for cyclic-gauche ethanol clusters: a) trimer, b) tetramer, c) pentamer, d) hexamer, e) heptamer and f) octamer, at the level M05-2X/6-31+G(d)

Table S1. Thermodynamic stability of ethanol clusters by comparing their corresponding solvation Gibbs free energies $\left(\Delta \mathrm{G}_{\text {solv }}^{*}(\mathrm{EtOH})_{\mathrm{x}}\right)$ as well as their solution phase Gibbs free energies $\left(\Delta G_{\mathrm{s}}(\mathrm{EtOH})_{x}\right)$.

Table S2. Average O-C-O bond distances obtained for the S6 $\left[\mathrm{OAc}(\mathrm{EtOH})_{j_{\text {first }}}\right]^{-}(\mathrm{EtOH})_{j_{\text {second }}}$ species at the M05-2X/6-31+G (d) level of theory with the SMD continuum solvation model.

Figure S3. Absolute errors in the computation of $\Delta_{\mathrm{r}} G_{\mathrm{s}, 1}^{*}$ for the $[\mathrm{Zn}(\mathrm{OAc})]^{+}$ species at the M05-2X/6-31+G(d) /SMD level of theory; by means of a) variant 1 (Eq.(7)) and b) variant 2 (Eq.(11)), for $j=1$ (white solid bars) $j=2$ (white dotted bars) $j=3$ (bars with horizontal lines)

Figure S4. Absolute errors in the computation of $\Delta_{\mathrm{r}} G_{\mathrm{s}, 1}^{*}$ for the $\left[\mathrm{Zn}(\mathrm{OAc})(\mathrm{EtOH})_{2}\right]^{+}$species at the M05-2X/6-31+G(d)/SMD level of theory; by means of a) variant 1 (Eq.(7)) and b) variant 2 (Eq.(11)), for $j=4+0$ (dotted bars) $j=4+1$ (solid bars) and $j=4+2$ (bars with diagonal lines)

Figure S5. Absolute errors in the computation of $\Delta_{\mathrm{r}} G_{\mathrm{s}, 1}^{*}$ for the $\left[\mathrm{Zn}(\mathrm{OAc})(\mathrm{EtOH})_{2}\right]^{+}(\mathrm{EtOH})$ species at the M05-2X/6-31+G(d)/SMD level of theory; by means of a) variant 1 (Eq.(7)) and b) variant 2 (Eq.(11)), for $j=4+0$ (dotted bars) $j=4+1$ (solid bars) and $j=4+2 j=6$ (bars with diagonal lines)

Figure S6. Absolute errors in the computation of $\Delta_{\mathrm{r}} G_{\mathrm{s}, 2}^{*}$ for the $\left[\mathrm{Zn}(\mathrm{OAc})_{2}\right]$ species at the M05-2X/6-31+G(d) /SMD level of theory; by means of a) variant 1 (Eq.(7)) and b) variant 2 (Eq.(11)), for $j=1$ (white solid bars) $j=2$ (white dotted bars) $j=3$ (bars with horizontal lines) 
Cartesian coordinates for ethanol clusters and for the $\left[\mathrm{OAc}(\mathrm{EtOH})_{j_{\text {first }}}\right]^{-}(\mathrm{EtOH})_{j_{\text {second }}},\left[\mathrm{Zn}(\mathrm{EtOH})_{k_{\mathrm{c}}}\right]^{2+}(\mathrm{EtOH})_{k_{\mathrm{f}}}$ and $\left[\mathrm{Zn}(\mathrm{OAc})_{n}\right]^{2-n}$ species in gas phase at the M05-2X/6-31+G(d) level of theory

Cartesian coordinates for ethanol clusters and for $\left[\mathrm{OAc}(\mathrm{EtOH})_{j_{\text {first }}}\right]^{-}(\mathrm{EtOH})_{j_{\text {second }}}$, $\left[\mathrm{Zn}(\mathrm{EtOH})_{k_{\mathrm{c}}}\right]^{2+}(\mathrm{EtOH})_{k_{\mathrm{f}}}$ and $\left[\mathrm{Zn}(\mathrm{OAc})_{n}\right]^{2-n}$ species in solution phase at the M05-2X/6-31+G(d) /SMD level of theory 
a)

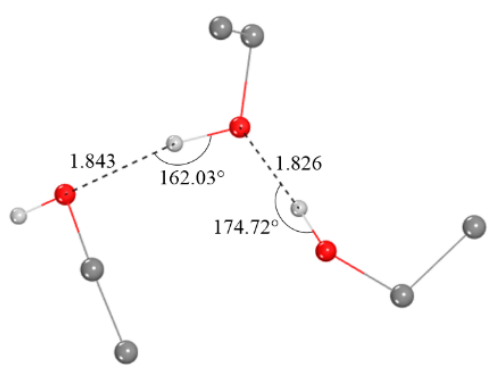

b)

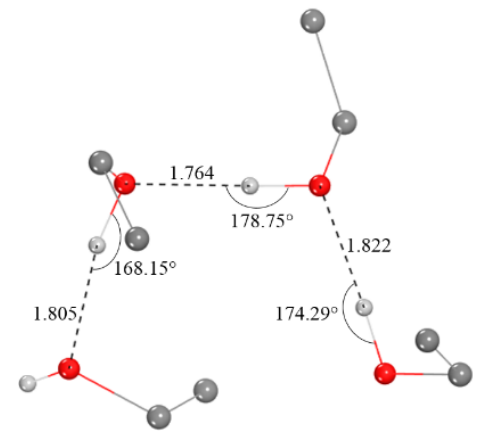

c)

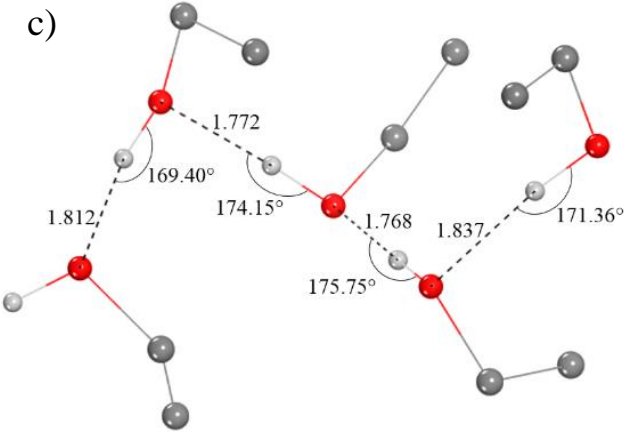

d)

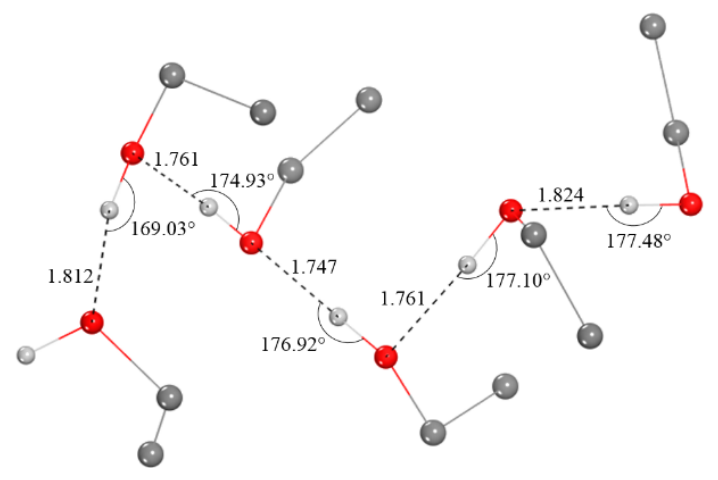

e)

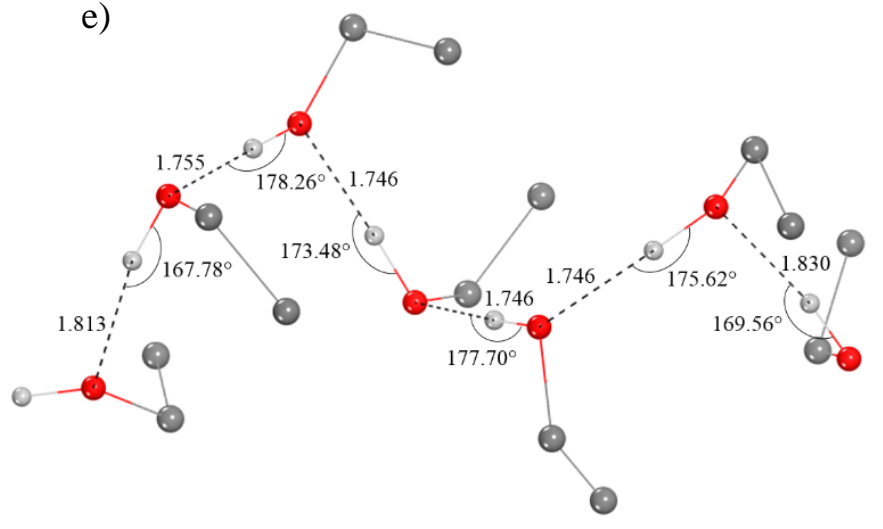

f)

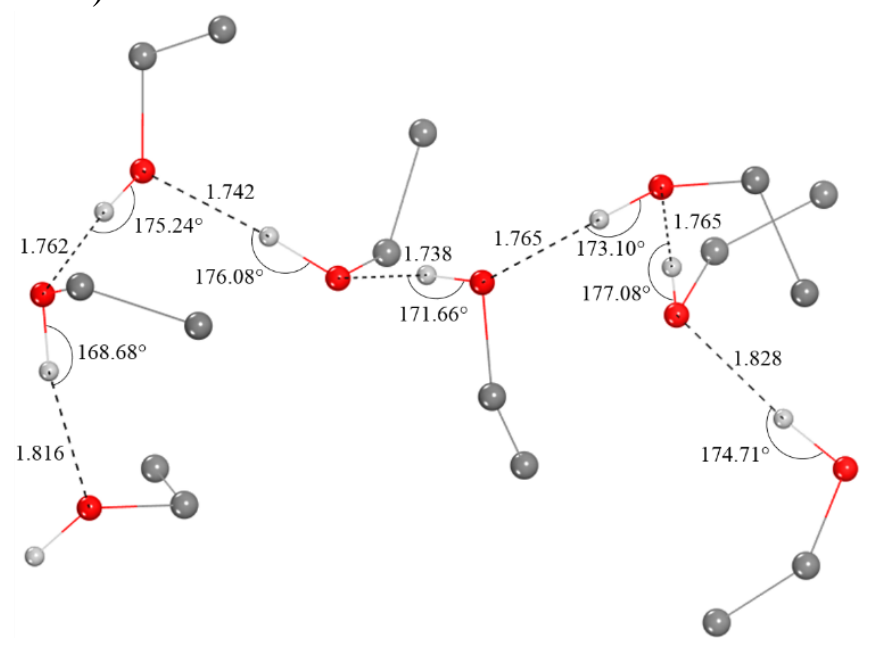

Figure S1. Relevant parameters of optimized geometries in the gas phase optimized for linear-gauche ethanol clusters: a) trimer, b) tetramer, c) pentamer, d) hexamer, e) heptamer and f) octamer, at the level M05-2X/6-31+G(d) 


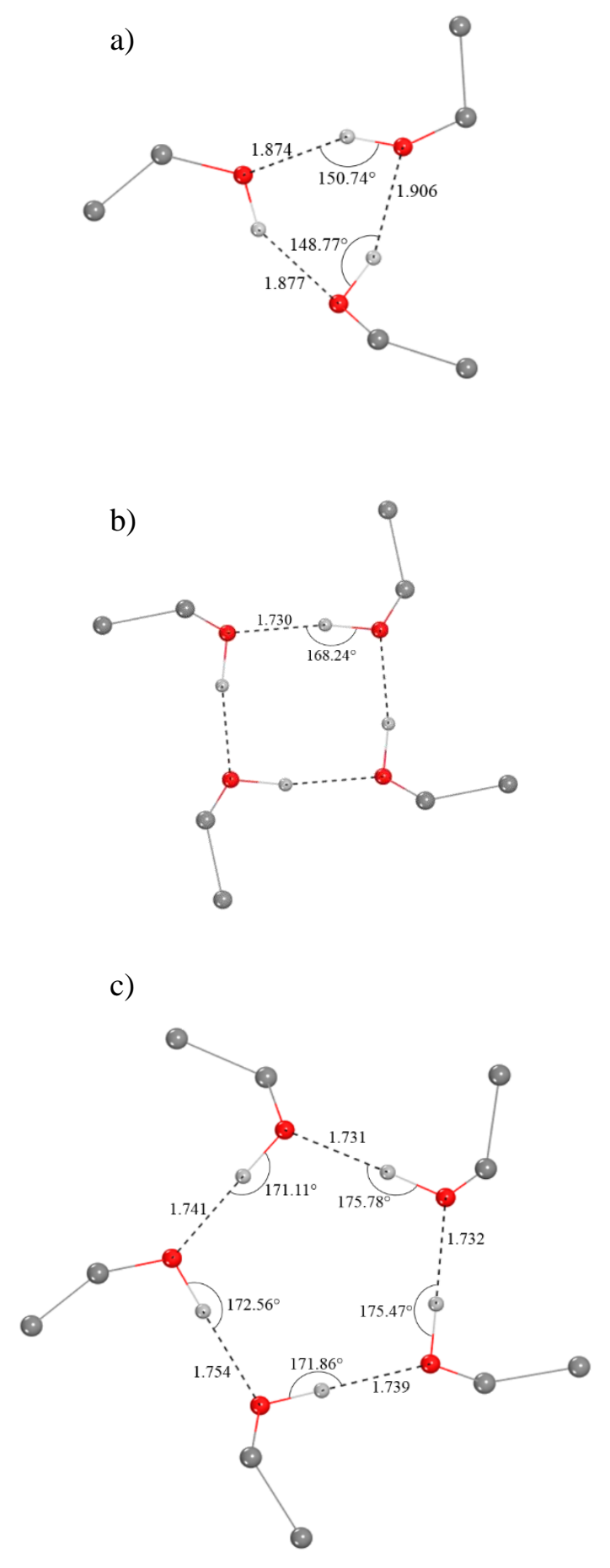

d)

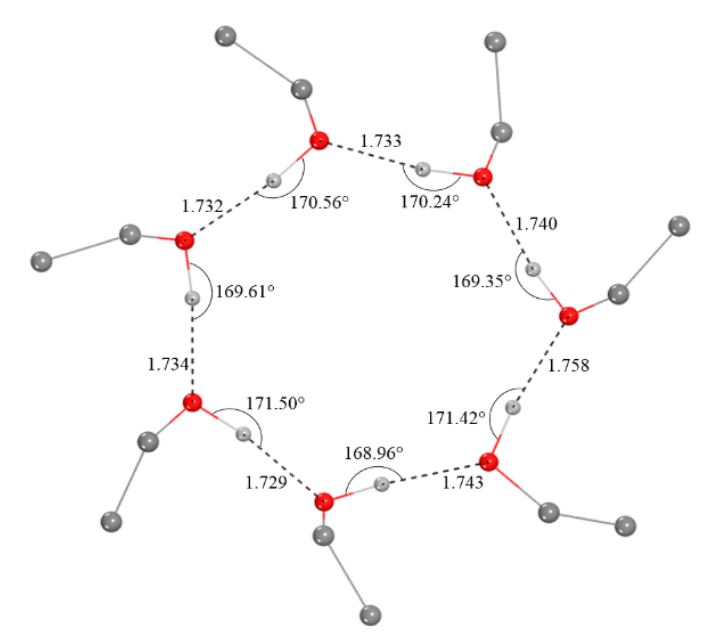

e)

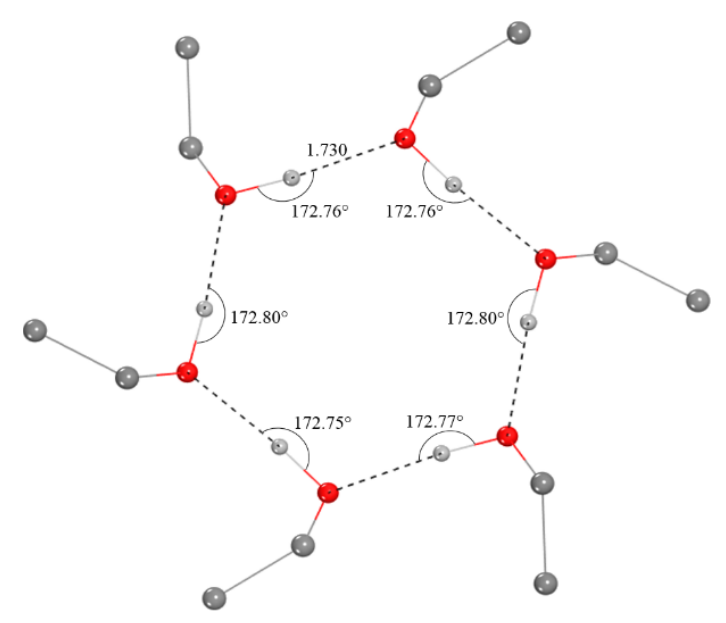

f)

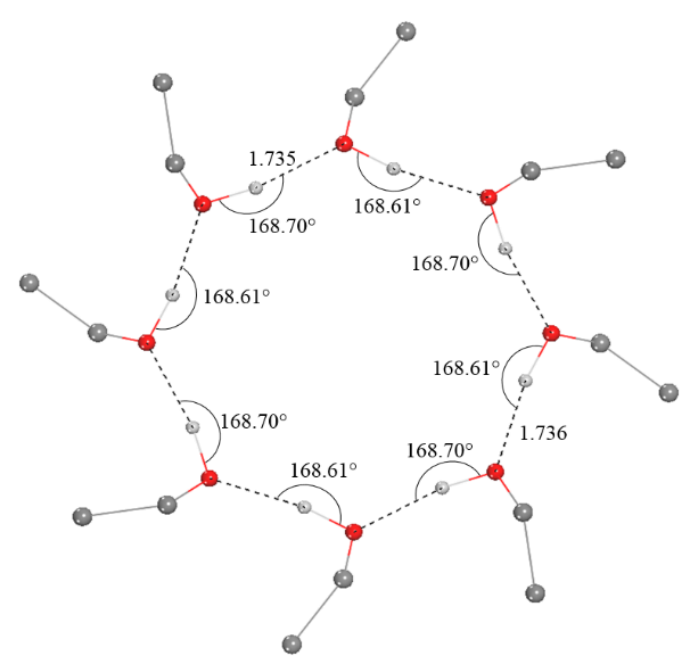

Figure S2. Relevant parameters of optimized geometries in the gas phase optimized for cyclic-gauche ethanol clusters: a) trimer, b) tetramer, c) pentamer, d) hexamer, e) heptamer and f) octamer, at the level M05-2X/6-31+G(d) 
Table S1. Thermodynamic stability of ethanol clusters by comparing their corresponding solvation Gibbs free energies $\left(\Delta \mathrm{G}_{\text {solv }}^{*}(\mathrm{EtOH})_{\mathrm{x}}\right)$ as well as their relative solution phase Gibbs free energies $\left(\Delta G_{\mathrm{s}}(\mathrm{EtOH})_{x}\right)$.

\begin{tabular}{ccc}
\hline$x$ & $\Delta G_{\text {solv }}^{*}(\mathrm{EtOH})_{x}$ & $\Delta G_{\mathrm{s}}(\mathrm{EtOH})_{x}^{\text {linear }}-\Delta G_{\mathrm{s}}(\mathrm{EtOH})_{x}^{\text {cyclic }}$ \\
\hline & Cyclic-gauche & \\
3 & -10.27 & 1.03 \\
4 & -11.17 & 3.16 \\
5 & -12.71 & 4.12 \\
6 & -13.62 & 4.62 \\
7 & -15.54 & 5.42 \\
8 & -16.84 & 5.45 \\
& Linear-gauche & \\
3 & -12.86 & \\
4 & -14.08 & \\
5 & -17.64 & \\
6 & -19.98 & \\
7 & -21.65 & \\
8 & -24.44 & \\
\hline
\end{tabular}

Table S2. Average O-C-O bond distances obtained for the $\left[\mathrm{OAc}(\mathrm{EtOH})_{j_{\text {frist }}}\right]^{-}(\mathrm{EtOH})_{j_{\text {second }}}$ species at the M052X/6-31+G (d) level of theory with the SMD continuum solvation model.

\begin{tabular}{cccc} 
Species & \multicolumn{2}{c}{ Ethanol } & \multirow{2}{*}{ Water $^{46}$} \\
\cline { 2 - 4 } Acetate & 1.256 & 1.264 & \\
{$[\mathrm{Ac}(\mathrm{EtOH})]^{-}$} & $1.267,1.249$ & $1.271,1.258$ & 1.265 \\
{$\left[\mathrm{Ac}(\mathrm{EtOH})_{2}\right]^{-}$} & $1.278,1.241$ & $1.278,1.253$ & $1.258,1.270$ \\
{$\left[\mathrm{Ac}(\mathrm{EtOH})_{3}\right]^{-}$} & $1.274,1.248$ & $1.272,1.259$ & $1.259,1.273$ \\
{$\left[\mathrm{Ac}(\mathrm{EtOH})_{4}\right]^{-}$} & $1.261,1.262$ & $1.267,1.265$ & $1.260,1.272$ \\
{$\left[\mathrm{Ac}(\mathrm{EtOH})_{4}\right]^{-}(\mathrm{EtOH})$} & $1.265,1.259$ & 1.266 & $1.262,1.274$ \\
\hline $\left.\mathrm{AtOH})_{4}\right]^{-}(\mathrm{EtOH})_{2}$ & $1.261,1.262$ & $1.267,1.265$ & $1.259,1.278$ \\
\hline
\end{tabular}


a)

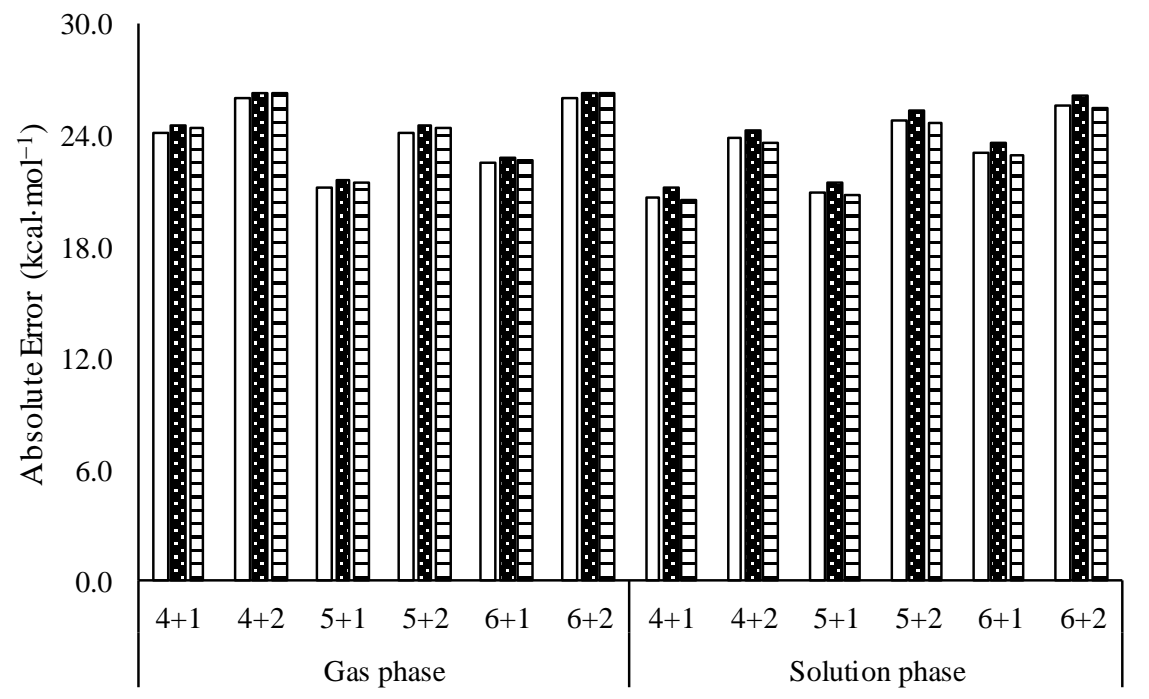

b)

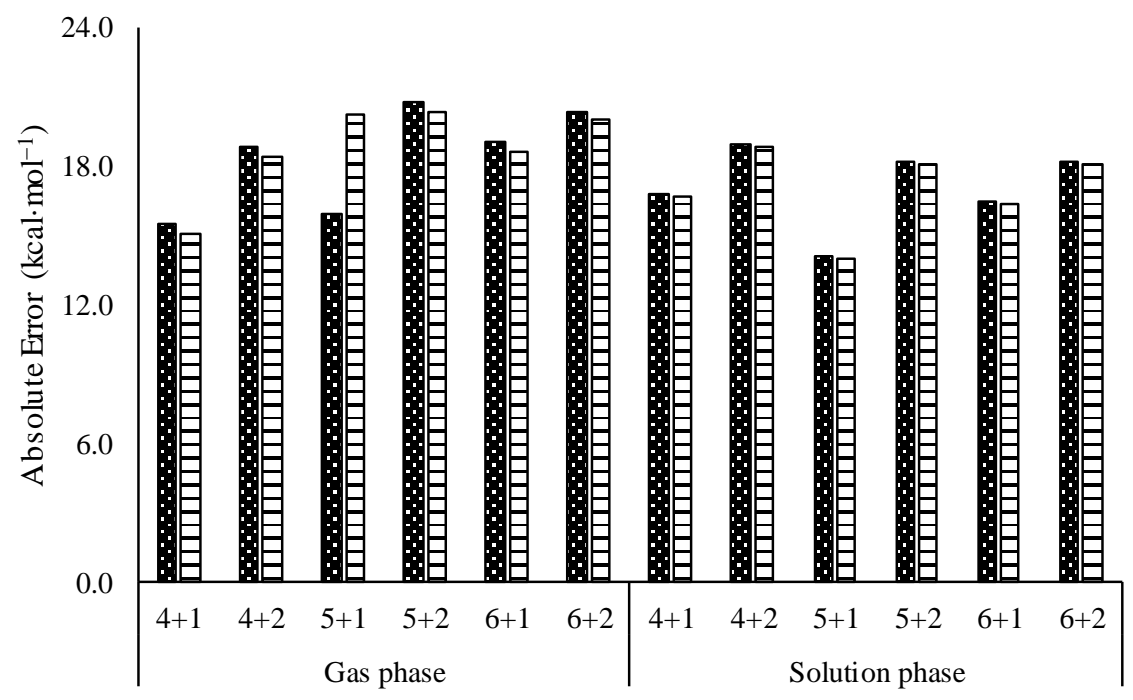

Figure S3. Absolute errors in the computation of $\Delta_{\mathrm{r}} G_{\mathrm{s}, 1}^{*}$ for the $[\mathrm{Zn}(\mathrm{OAc})]^{+}$species at the M05-2X/6-31+G(d) /SMD level of theory; by means of a) variant 1 (Eq.(7)) and b) variant 2 (Eq.(11)), for $j=1$ (white solid bars) $j=2$ (white dotted bars) $j=3$ (bars with horizontal lines) 
a)

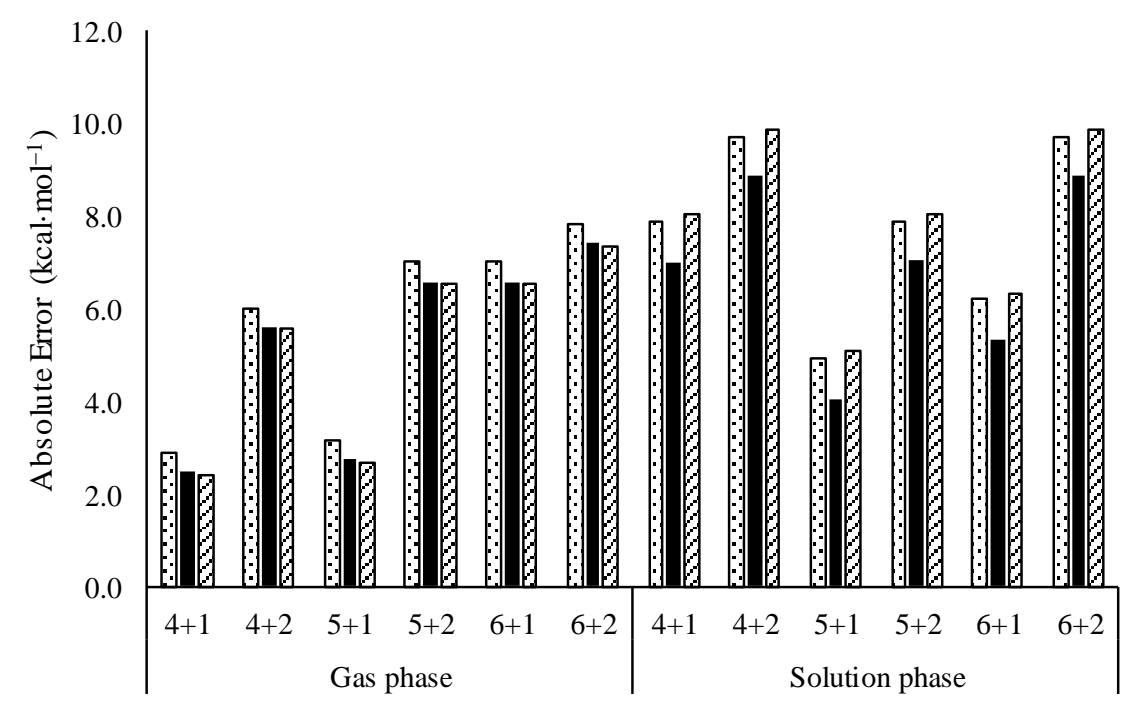

b)

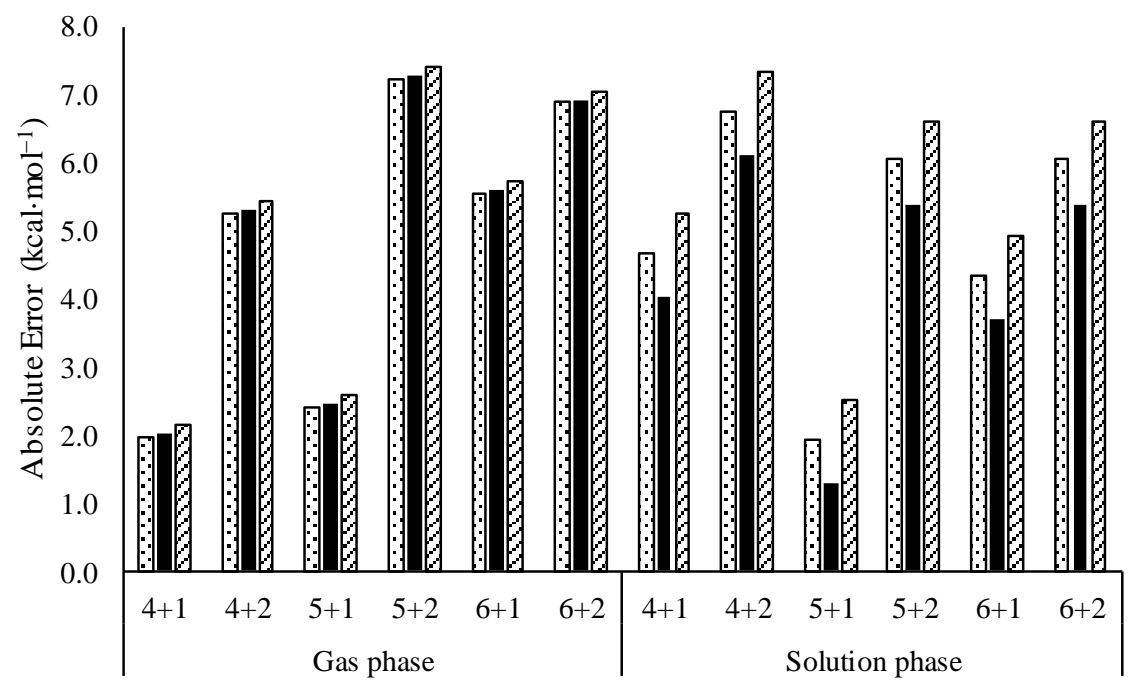

Figure S4. Absolute errors in the computation of $\Delta_{\mathrm{r}} G_{\mathrm{s}, 1}^{*}$ for the $\left[\mathrm{Zn}(\mathrm{OAc})(\mathrm{EtOH})_{2}\right]^{+}$ species at the M05-2X/6-31+G(d) /SMD level of theory; by means of a) variant 1 (Eq.(7)) and b) variant 2 (Eq.(11)), for $j=4+0$ (dotted bars) $j=4+1$ (solid bars) and $j=4+2$ (bars with diagonal lines). 
a)

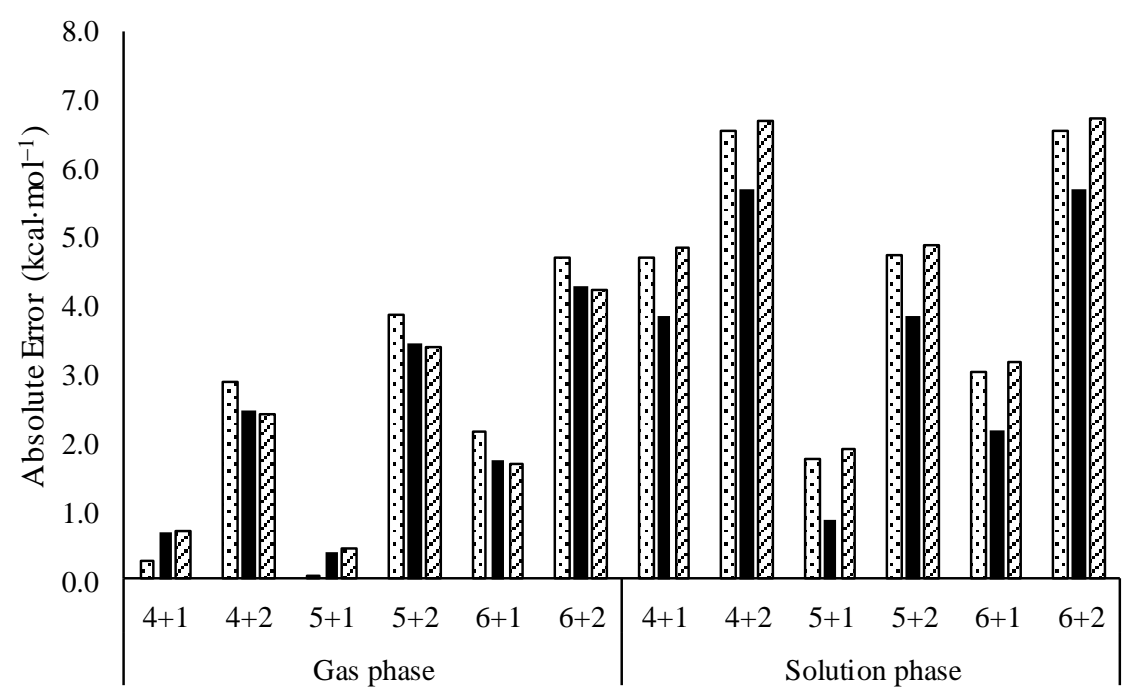

b)

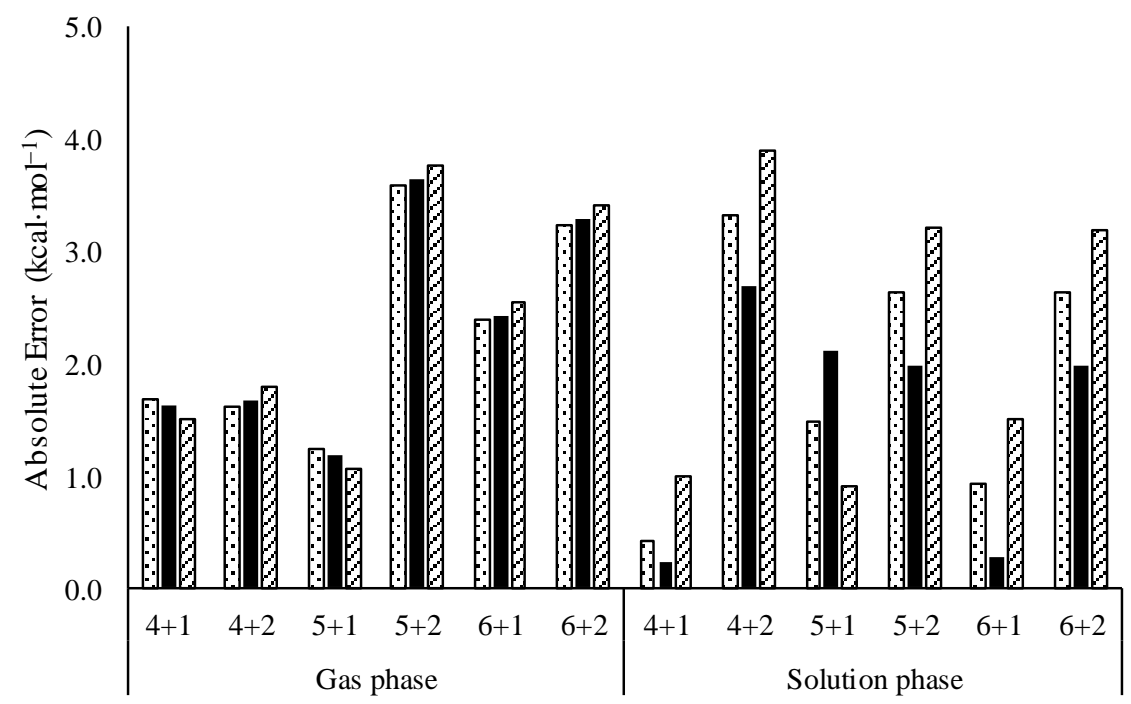

Figure S5. Absolute errors in the computation of $\Delta_{\mathrm{r}} G_{\mathrm{s}, 1}^{*}$ for the $\left[\mathrm{Zn}(\mathrm{OAc})(\mathrm{EtOH})_{2}\right]^{+}(\mathrm{EtOH})$ species at the M05-2X/6-31+G(d)/SMD level of theory; by means of a) variant 1 (Eq.(7)) and b) variant 2 (Eq.(11)), for $j=4+0$ (dotted bars) $j=4+1$ (solid bars) and $j=4+2$ (bars with diagonal lines). 
a)

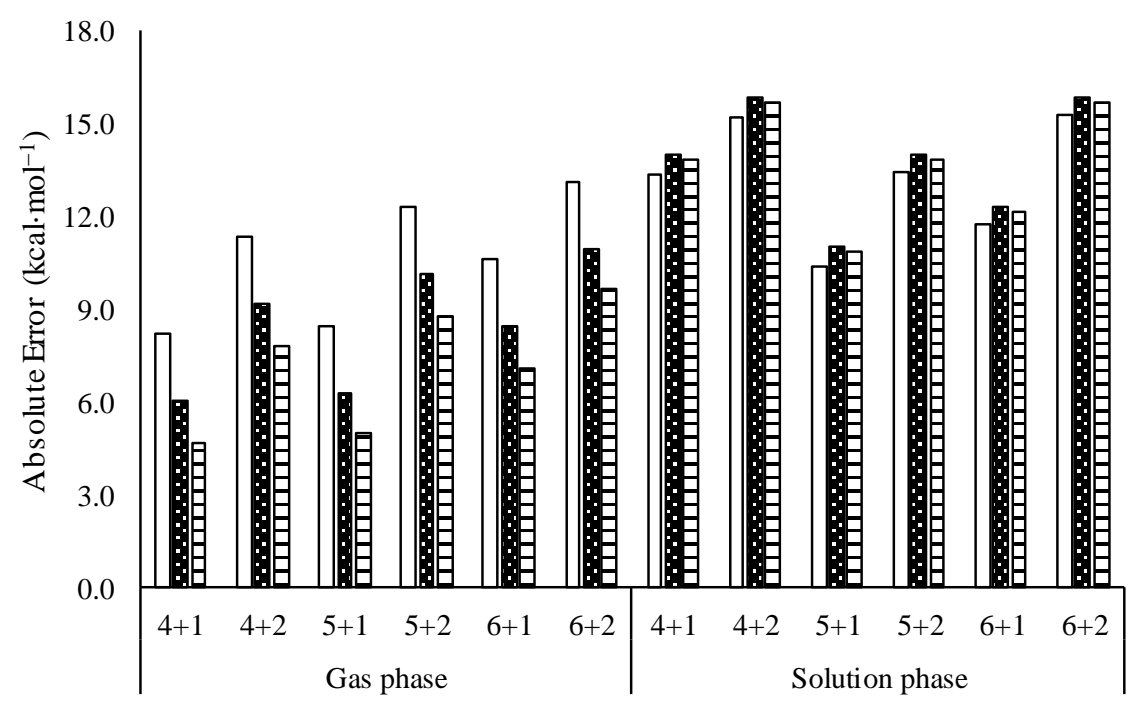

b)

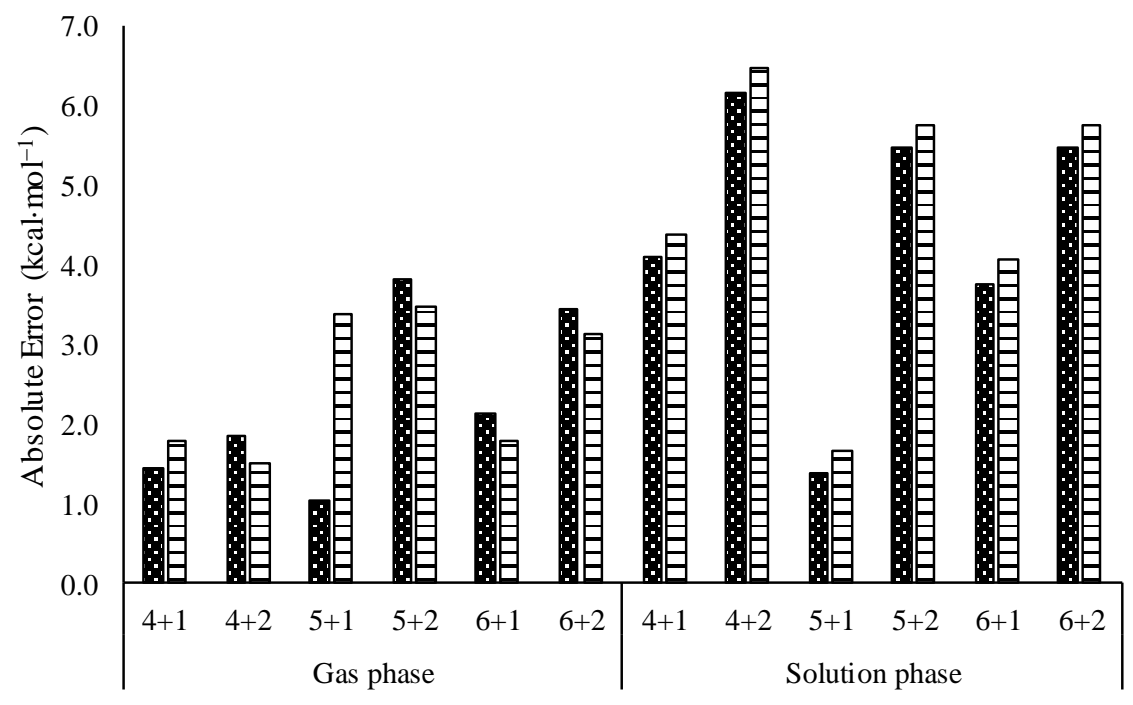

Figure S6. Absolute errors in the computation of $\Delta_{\mathrm{r}} G_{\mathrm{s}, 2}^{*}$ for the $\left[\mathrm{Zn}(\mathrm{OAc})_{2}\right]$ species at the M05-2X/6-31+G(d)/SMD level of theory; by means of a) variant 1 (Eq.(7)) and b) variant 2 (Eq. (11)), for $j=1$ (white solid bars) $j=2$ (white dotted bars) $j=3$ (bars with horizontal lines). 
Optimized geometries of Zinc complexes in gas phase at the M05-2X/6-31 +G(d) level of theory.

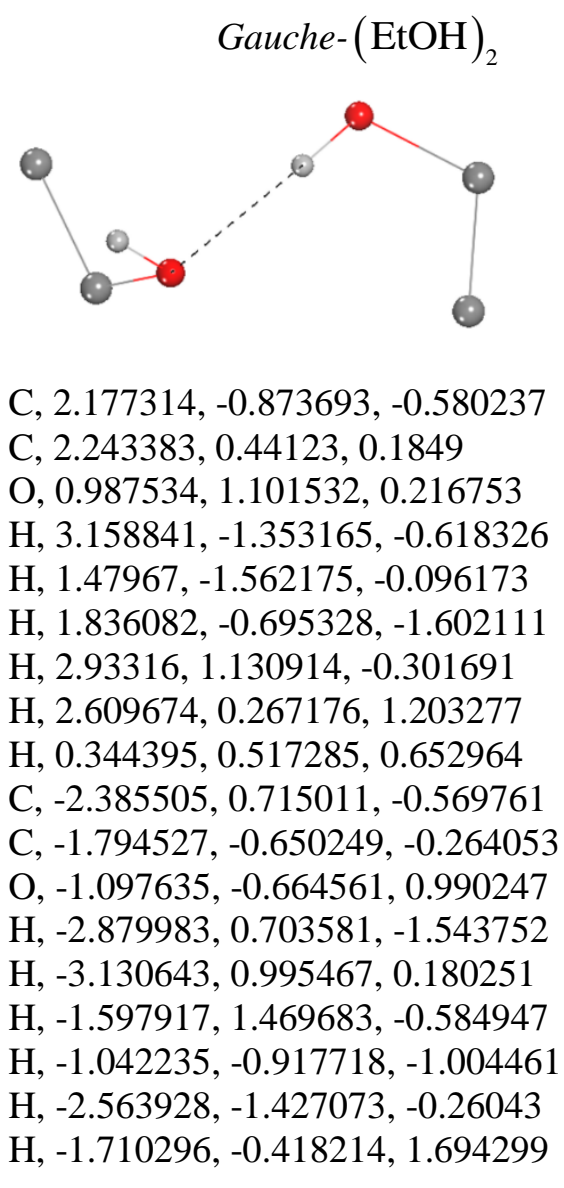

Linear-gauche $(\mathrm{EtOH})_{3}$

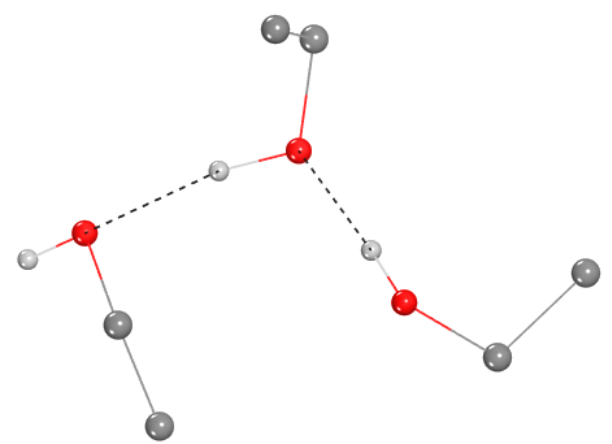

C, $-1.700647,-2.231139,-0.259797$

C, $-2.193068,-1.034697,0.532598$

$\mathrm{O},-2.585092,0.051217,-0.327919$
$\mathrm{H},-3.259674,-0.260852,-0.943689$

$\mathrm{H},-2.500493,-2.655725,-0.873937$

H, $-0.87346,-1.938047,-0.907933$

$\mathrm{H},-1.340521,-3.006618,0.418734$

H, $-3.034431,-1.30059,1.177535$

$\mathrm{H},-1.391782,-0.636899,1.152171$

$\mathrm{H},-0.999573,0.787173,-0.911173$

O, $-0.054874,1.025234,-0.977267$

C, $0.126277,2.34621,-0.463848$

C, $-0.330623,2.460656,0.982767$

H, -0.406473, 3.060991, - 1.09811

H, 1.193464, 2.551804, - 0.5493

H, $0.222374,1.759082,1.610523$

H, -1.397013, 2.237229, 1.064837

H, $-0.162377,3.473364,1.357512$

$\mathrm{H}, 0.801693,-0.152366,0.12431$

O, 1.194228, $-0.768406,0.773488$

C, 2.296048, $-1.415293,0.158003$

C, 3.341392, $-0.419736,-0.326122$

$\mathrm{H}, 1.95487,-2.038023,-0.678671$

H, 2.720712, - 2.078176, 0.912565

$\mathrm{H}, 3.693799,0.188564,0.5091$

H, 2.912066, 0.243843, - 1.081327

$\mathrm{H}, 4.196449,-0.935118,-0.771166$

Linear-gauche $(\mathrm{EtOH})_{4}$

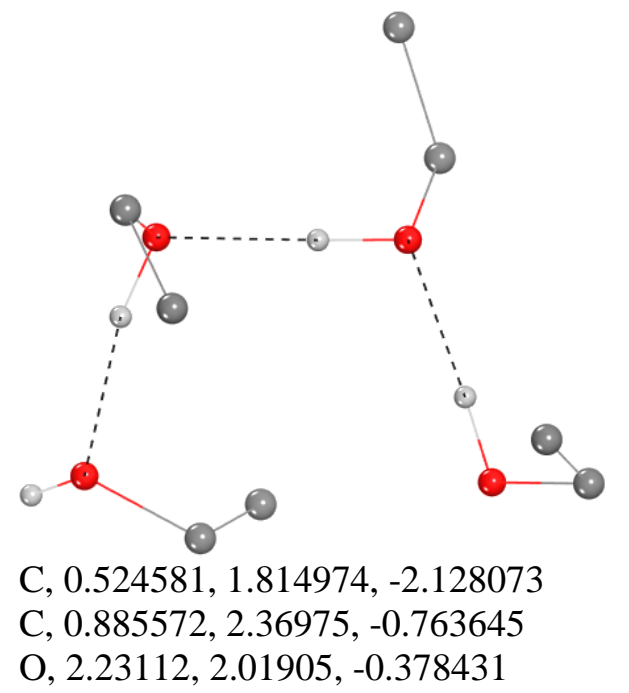


H, 2.844864, 2.339797, -1.050807

H, 1.150383, 2.251911, -2.912257

H, 0.653751, 0.73046, -2.140783

H, $-0.521313,2.033397,-2.346714$

H, $0.779394,3.456677,-0.7342$

$\mathrm{H}, 0.240575,1.944428,0.001837$

$\mathrm{H}, 2.21171,0.254227,0.001725$

$\mathrm{O}, 2.004817,-0.671395,0.243897$

C, $2.20786,-0.838054,1.649213$

C, $1.333699,0.104509,2.461681$

H, 3.265556, $-0.688279,1.883472$

H, 1.959047, $-1.877989,1.862198$

H, 0.279931, $-0.055852,2.223864$

$\mathrm{H}, 1.58618,1.144366,2.242161$

$\mathrm{H}, 1.478251,-0.065461,3.531403$

H, $0.33045,-1.067727,-0.144041$

O, $-0.596801,-1.309054,-0.361558$

C, $-0.574575,-2.283678,-1.40089$

C, $0.145523,-3.548082,-0.959743$

H, $-0.10078,-1.865365,-2.295545$

H, $-1.616832,-2.494466,-1.641018$

H, $-0.340556,-3.968307,-0.077452$

H, $1.18534,-3.323175,-0.711483$

H, $0.136032,-4.296389,-1.756065$

H, $-1.603896,0.203199,-0.495107$

$\mathrm{O},-2.117343,1.034948,-0.47528$

C, $-3.26807,0.823226,0.326996$

C, $-2.907528,0.508581,1.772702$

H, $-3.881124,0.016251,-0.091504$

$\mathrm{H},-3.850868,1.743423,0.271504$

H, $-2.308662,1.318987,2.194124$

H, $-2.326767,-0.415266,1.824898$

H, $-3.807394,0.3854,2.381327$

Linear-gauche $(\mathrm{EtOH})_{5}$

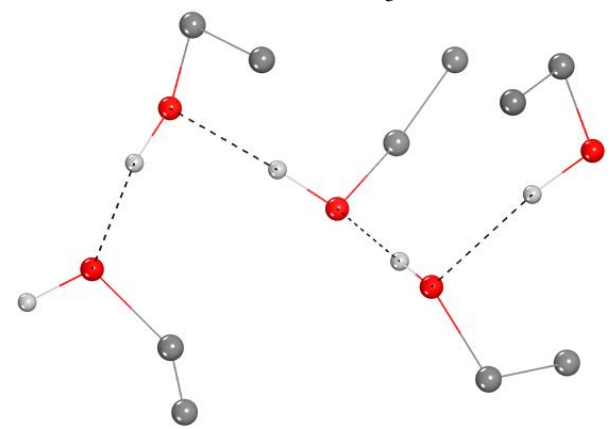

C, $-2.81395,2.007274,-0.92055$

C, $-2.632027,0.729458,-1.719318$

O, $-3.428197,-0.348858,-1.192491$

H, $-4.359076,-0.09449,-1.21363$
H, $-3.856704,2.337975,-0.938348$

$\mathrm{H},-2.506488,1.86142,0.116288$

H, $-2.203805,2.804409,-1.350514$

H, $-2.888977,0.876942,-2.771279$

H, $-1.600526,0.387264,-1.660582$

H, $-2.772218,-1.000522,0.365513$

O, $-2.256589,-1.312978,1.135716$

C, $-1.621151,-2.543948,0.784595$

C, $-0.679216,-2.37724,-0.397318$

H, $-2.383645,-3.298441,0.571405$

H, $-1.078023,-2.86259,1.674328$

H, $0.048039,-1.588264,-0.19541$

H, $-1.23674,-2.108039,-1.29717$

H, $-0.13844,-3.306436,-0.595158$

$\mathrm{H},-1.19043,0.036557,1.563421$

O, $-0.599098,0.809528,1.703178$

C, 0.18886, 0.591916, 2.874811

C, $1.255268,-0.471309,2.659576$

H, $-0.469525,0.322122,3.705344$

$\mathrm{H}, 0.649129,1.551075,3.112581$

H, 1.926539, $-0.193271,1.844217$

H, $0.795339,-1.433152,2.418183$

H, 1.850912, $-0.601048,3.566921$

$\mathrm{H}, 0.250241,1.04507,0.171133$

O, 0.702381, 1.108661, -0.69995

C, 1.126772, 2.456501, -0.905214

C, 1.984294, 2.964846, 0.244078

H, 0.251977, 3.099942, -1.044518

H, 1.695106, 2.451943, -1.835544

H, 2.855213, 2.321197, 0.380669

$\mathrm{H}, 1.406895,2.978652,1.171847$

H, 2.32763, 3.982661, 0.043023

H, 2.282984, 0.17607, -0.608809

O, 3.178773, $-0.19775,-0.499191$

C, $3.13429,-1.581955,-0.798818$

C, $2.625256,-1.846549,-2.209419$

H, $2.511813,-2.113135,-0.066762$

H, 4.155004, $-1.949598,-0.688206$

H, 3.268814, $-1.350497,-2.938185$

H, 1.610361, $-1.458159,-2.328545$

H, 2.61006, -2.918443, -2.424837 
Linear-gauche $(\mathrm{EtOH})_{6}$

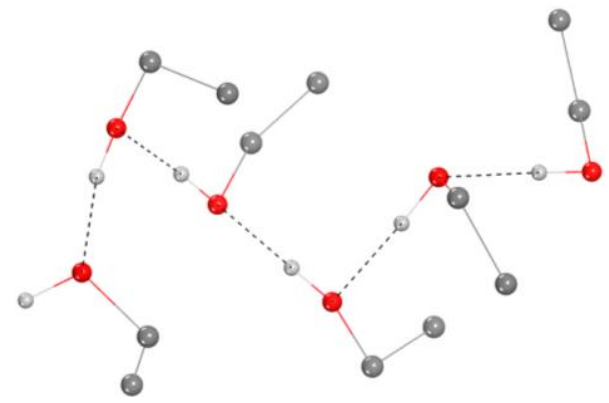

C, 3.537753, 1.674668, - 1.282099

C, 3.453447, 1.579898, 0.229801

O, 4.262205, 0.507441, 0.74849

$\mathrm{H}, 5.176766,0.63502,0.467443$

$\mathrm{H}, 4.559804,1.890623,-1.60765$

H, 3.20821, $0.742709,-1.744673$

H, 2.891462, 2.479779, - 1.637277

$\mathrm{H}, 3.758384,2.51533,0.705693$

$\mathrm{H}, 2.43489,1.35275,0.537821$

H, 3.479865, -1.104552, 0.477317

$\mathrm{O}, 2.898377,-1.878275,0.336986$

C, 2.389193, -2.301843, 1.603873

C, $1.56151,-1.219531,2.278366$

H, 3.222936, -2.607907, 2.242115

$\mathrm{H}, 1.78417,-3.185639,1.401408$

$\mathrm{H}, 0.720615,-0.921495,1.648767$

H, 2.180067, $-0.341918,2.48205$

$\mathrm{H}, 1.163033,-1.582221,3.229481$

$\mathrm{H}, 1.806199,-1.432267,-0.970213$

O, 1.222268, -1.100909, -1.689539

C, $0.3039,-2.130783,-2.0575$

C, $-0.715073,-2.402412,-0.961428$

$\mathrm{H}, 0.86073,-3.038106,-2.307613$

$\mathrm{H},-0.190395,-1.78395,-2.965323$

$\mathrm{H},-1.246181,-1.487595,-0.692992$

H, $-0.225494,-2.781808,-0.061341$

H, $-1.446415,-3.145579,-1.290142$

H, $0.56912,0.42614,-1.14862$

O, 0.223295, 1.279068, -0.796347

C, $-0.341633,2.029896,-1.87272$

C, $-1.614017,1.393443,-2.41019$

H, $0.404319,2.135367,-2.66666$

H, $-0.549844,3.022635,-1.4724$

H, -2.375926, 1.322914, -1.631246

H, $-1.40661,0.389773,-2.790645$

H, -2.018229, 1.989057, -3.232745

$\mathrm{H},-0.882821,0.793716,0.48494$

O, -1.487696, 0.475506, 1.191136

C, $-1.378554,1.349558,2.312099$

C, $-1.760132,2.777628,1.951005$
$\mathrm{H},-0.358933,1.310187,2.710583$

H, - 2.052119, 0.950624, 3.070821

$\mathrm{H},-2.776315,2.807685,1.552797$

$\mathrm{H},-1.077875,3.17316,1.194175$

$\mathrm{H},-1.708018,3.425373,2.829707$

$\mathrm{H},-3.196748,0.511271,0.556121$

$\mathrm{O},-4.096548,0.537249,0.176455$

C, $-4.368399,-0.733161,-0.389444$

C, $-4.255908,-1.853666,0.635888$

$\mathrm{H},-3.694668,-0.928988,-1.234631$

$\mathrm{H},-5.383035,-0.680064,-0.785973$

H, $-4.954593,-1.681002,1.456629$

H, $-3.244257,-1.891175,1.047761$

$\mathrm{H},-4.479817,-2.822664,0.181164$

Linear-gauche $(\mathrm{EtOH})_{7}$

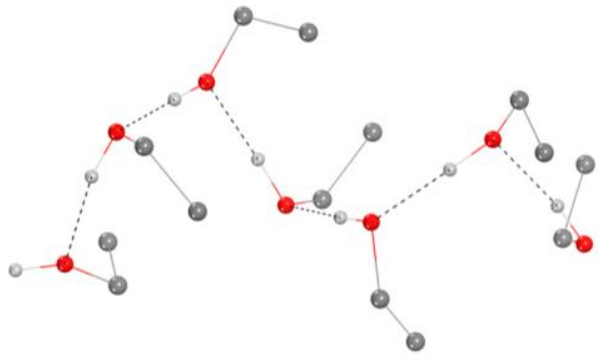

H, 5.634422, 1.31946, 1.156647

O, 4.802736, 0.835379, 1.231954

C, 3.710744, 1.772959, 1.271213

C, 3.701825, 2.685497, 0.059172

$\mathrm{H}, 2.807331,1.166728,1.29562$

H, 3.768046, 2.345201, 2.200532

$\mathrm{H}, 3.620758,2.103635,-0.860606$

H, 4.611359, 3.292155, 0.015822

$\mathrm{H}, 2.845476,3.360383,0.112507$

$\mathrm{H}, 4.433327,-0.502494,0.065176$

O, 4.036361, $-1.16631,-0.533185$

C, $3.732675,-2.343751,0.218324$

C, $2.780167,-2.059147,1.368539$

$\mathrm{H}, 4.663595,-2.785942,0.584526$

$\mathrm{H}, 3.288687,-3.042557,-0.490999$

$\mathrm{H}, 1.840309,-1.640876,1.002737$

H, 3.23333, -1.354045, 2.06992

H, 2.554961, -2.980766, 1.911593

H, 2.810091, $-0.356055,-1.492315$

$\mathrm{O}, 2.13207,0.124064,-2.018701$

C, 1.534607, $-0.794995,-2.935204$

C, $0.505476,-1.688548,-2.259127$

H, 2.321498, -1.392243, -3.404235

H, 1.066546, -0.192066, -3.713917 
$\mathrm{H},-0.306913,-1.094967,-1.836425$

$\mathrm{H}, 0.96198,-2.26261,-1.449178$

$\mathrm{H}, 0.078964,-2.390233,-2.981057$

$\mathrm{H}, 1.141731,0.969582,-0.856175$

$\mathrm{O}, 0.616151,1.370779,-0.124739$

C, $-0.217216,2.404571,-0.650536$

C, $-1.130848,1.900073,-1.756155$

H, 0.410962, 3.224594, -1.012995

H, $-0.802025,2.776995,0.191337$

$\mathrm{H},-1.750422,1.073669,-1.402565$

H, $-0.542727,1.55398,-2.610059$

$\mathrm{H},-1.788585,2.702184,-2.101091$

H, $-0.151986,0.011808,0.658326$

$\mathrm{O},-0.587138,-0.772414,1.064645$

C, $-0.698149,-0.562979,2.47271$

C, $-1.498581,0.686842,2.803147$

$\mathrm{H}, 0.305385,-0.510098,2.908324$

H, -1.189186, -1.451123, 2.871489

$\mathrm{H},-2.504885,0.624752,2.383465$

$\mathrm{H},-1.000249,1.572837,2.400734$

$\mathrm{H},-1.582179,0.809344,3.8861$

$\mathrm{H},-2.045311,-0.99651,0.130666$

$\mathrm{O},-2.851795,-1.057422,-0.429569$

C, $-3.161576,-2.431469,-0.642207$

C, $-3.445602,-3.153317,0.666615$

$\mathrm{H},-2.33783,-2.914996,-1.177891$

$\mathrm{H},-4.039045,-2.446244,-1.289045$

$\mathrm{H},-4.26282,-2.662734,1.199309$

H, -2.559339, -3.143438, 1.305895

$\mathrm{H},-3.721916,-4.194526,0.4824$

$\mathrm{H},-4.113975,-0.093215,0.4783$

O, $-4.666131,0.551071,0.961882$

C, $-4.477748,1.8122,0.344606$

C, $-4.972074,1.824519,-1.095773$

$\mathrm{H},-3.417867,2.100028,0.379079$

H, $-5.033921,2.53382,0.944535$

H, $-6.032639,1.568564,-1.128257$

H, -4.421208, 1.091516, -1.689996

H, -4.833953, 2.810594, -1.548474
Linear-gauche $(\mathrm{EtOH})_{8}$

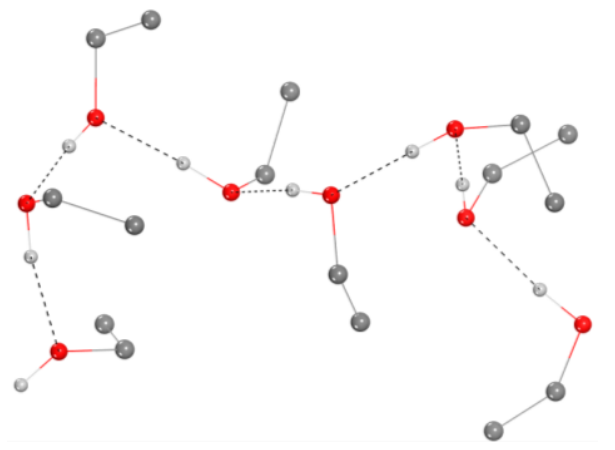

$\mathrm{H},-4.561868,-3.470068,-0.973765$

$\mathrm{O},-4.103169,-2.837575,-0.406882$

C, $-2.768846,-2.632983,-0.90787$

C, $-2.768749,-2.15613,-2.347668$

$\mathrm{H},-2.32857,-1.87598,-0.262317$

H, $-2.200524,-3.559726,-0.793429$

$\mathrm{H},-3.316018,-1.216154,-2.439266$

$\mathrm{H},-3.224465,-2.898531,-3.009666$

$\mathrm{H},-1.742643,-1.988037,-2.680376$

$\mathrm{H},-4.917721,-1.272186,0.022936$

O, $-5.178541,-0.371685,0.301751$

C, $-5.12903,-0.301996,1.728028$

C, $-3.746198,-0.631083,2.267726$

H, $-5.879107,-0.977291,2.149216$

$\mathrm{H},-5.417546,0.716992,1.986575$

$\mathrm{H},-2.99322,0.034599,1.839432$

$\mathrm{H},-3.482414,-1.662779,2.02391$

$\mathrm{H},-3.722093,-0.520879,3.354668$

$\mathrm{H},-4.237935,0.770363,-0.654804$

O, -3.654616, 1.350764, - 1.193404

C, $-3.902771,2.714791,-0.854401$

C, $-3.332773,3.075922,0.509234$

H, $-4.978816,2.906312,-0.890192$

$\mathrm{H},-3.43336,3.311762,-1.636713$

$\mathrm{H},-2.260495,2.871341,0.543406$

$\mathrm{H},-3.815617,2.494088,1.297548$

$\mathrm{H},-3.491269,4.135794,0.723216$

H, -2.038471, 0.731856, -0.989964

O, $-1.152576,0.322769,-0.858854$

C, $-0.27229,0.759559,-1.899215$

C, $-0.000103,2.253379,-1.816248$

$\mathrm{H},-0.710091,0.499148,-2.867878$

$\mathrm{H}, 0.653735,0.197692,-1.775618$

H, $0.481263,2.504564,-0.868848$

$\mathrm{H},-0.931701,2.819032,-1.900514$

H, $0.658384,2.565106,-2.630776$

$\mathrm{H},-0.508084,0.401177,0.753044$

$\mathrm{O},-0.051928,0.343501,1.623817$ 
C, $0.134724,-1.0371,1.933089$

C, 0.939921, $-1.758216,0.862488$

H, $-0.8407,-1.514985,2.070578$

H, 0.652141, -1.065154, 2.892699

H, 1.90072, -1.264057, 0.708092

H, 0.402322, - $1.754616,-0.088551$

H, 1.124976, -2.796149, 1.152647

$\mathrm{H}, 1.412926,1.291139,1.356962$

$\mathrm{O}, 2.221871,1.786412,1.094718$

C, 3.068884, 1.942638, 2.23349

C, 3.718144, 0.631414, 2.650019

H, 2.489217, 2.3695, 3.056992

H, 3.829139, 2.670215, 1.948373

$\mathrm{H}, 4.321614,0.219761,1.838226$

$\mathrm{H}, 2.956504,-0.102671,2.926115$

H, 4.366909, 0.786616, 3.516076

$\mathrm{H}, 2.86598,0.914872,-0.298935$

O, 3.208443, 0.386689, - -1.054726

C, 3.866477, 1.264002, - 1.967755

C, 4.965444, 2.067611, -1.288472

$\mathrm{H}, 3.131688,1.931258,-2.42973$

$\mathrm{H}, 4.282556,0.626413,-2.748216$

H, 5.692722, 1.397655, - 0.825663

$\mathrm{H}, 4.540621,2.713101,-0.515855$

$\mathrm{H}, 5.482685,2.700186,-2.014191$

$\mathrm{H}, 4.512744,-0.742011,-0.449629$

O, 5.265095, -1.274246, -0.124377

C, 4.770903, $-2.524459,0.322148$

C, $4.041729,-3.279779,-0.781232$

$\mathrm{H}, 4.108033,-2.388824,1.18706$

H, 5.638682, $-3.091535,0.661129$

$\mathrm{H}, 4.713593,-3.45006,-1.624491$

H, 3.185092, - $2.701427,-1.136829$

H, 3.679066, $-4.245873,-0.419195$

Cyclic-gauche $(\mathrm{EtOH})_{3}$

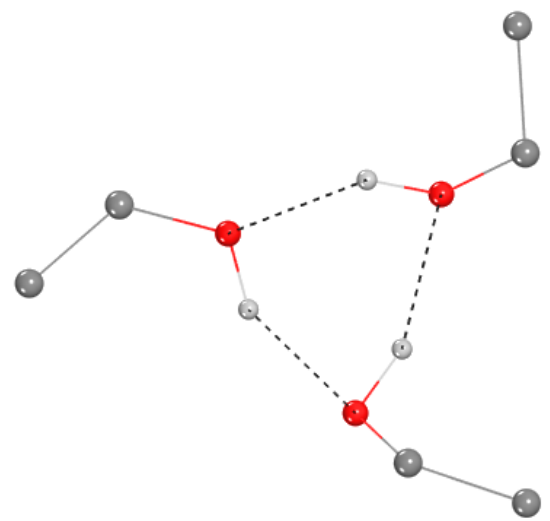

C, $2.095805,-2.706209,0.139158$

C, $0.688166,-2.230515,0.458688$
$\mathrm{O}, 0.062172,-1.639839,-0.679265$

H, 0.614877, $-0.881661,-0.954946$

H, 2.074052, $-3.420317,-0.685322$

$\mathrm{H}, 2.728214,-1.86304,-0.151435$

H, 2.54819, -3.186106, 1.010211

$\mathrm{H}, 0.053415,-3.06742,0.748418$

$\mathrm{H}, 0.704896,-1.514169,1.288518$

H, -1.21066, -0.411186, -0.053014

$\mathrm{O},-1.453489,0.487729,0.248858$

C, $-2.745455,0.827798,-0.248865$

C, $-3.816687,-0.102475,0.296473$

$\mathrm{H},-2.740623,0.810551,-1.343384$

H, $-2.927359,1.853079,0.072029$

$\mathrm{H},-3.818153,-0.075892,1.387215$

$\mathrm{H},-3.632476,-1.130628,-0.024672$

$\mathrm{H},-4.804421,0.193824,-0.064584$

$\mathrm{H}, 0.198021,1.124274,-0.368335$

$\mathrm{O}, 1.08332,0.954031,-0.747807$

C, $2.081524,1.441485,0.143483$

C, $1.988253,2.948422,0.320535$

H, 3.038236, 1.166545, -0.300673

$\mathrm{H}, 1.999699,0.936867,1.112867$

$\mathrm{H}, 1.029523,3.223137,0.76711$

H, 2.074507, 3.445587, - 0.646709

$\mathrm{H}, 2.784401,3.310169,0.97559$

Cyclic-gauche $(\mathrm{EtOH})_{4}$

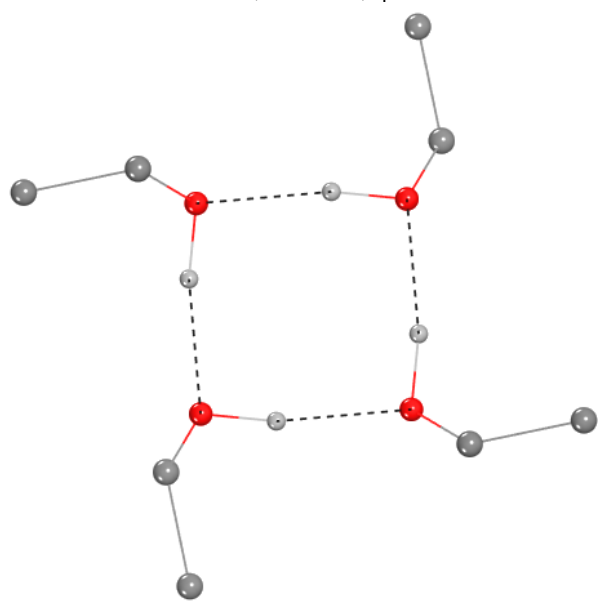

C, $-3.911302,-0.161345,0.963906$

C, $-2.693351,0.731903,1.13299$

$\mathrm{O},-1.821066,0.636151,0.008141$

$\mathrm{H},-1.500496,-0.293198,-0.056914$

$\mathrm{H},-4.45126,0.106496,0.054292$

H, -3.605597, $-1.207894,0.892438$

$\mathrm{H},-4.585948,-0.059733,1.817362$

H, -2.987546, 1.779558, 1.19985 
H, -2.15066, 0.471383, 2.047313

H, $-0.293196,1.500511,0.056924$

O, $0.636153,1.821055,-0.008248$

C, $0.731878,2.692979,-1.13338$

C, $-0.161296,3.911029,-0.964622$

$\mathrm{H}, 0.471269,2.150012,-2.047514$

H, 1.779544, 2.987099, -1.200402

H, 0.10663, 4.451257, -0.055193

H, $-1.207855,3.605398,-0.892993$

H, $-0.059705,4.585407,-1.818293$

H, 1.500485, $0.293173,-0.056763$

$\mathrm{O}, 1.821048,-0.636165,0.008497$

C, $2.69319,-0.731717,1.133474$

C, 3.911171, 0.161487, 0.964376

H, 2.987366, -1.779362, 1.200567

H, 2.150387, $-0.47102,2.04768$

H, 3.605486, 1.208026, 0.892677

H, 4.45124, $-0.106528,0.054879$

H, 4.585711, 0.060026, 1.817935

H, 0.29317, $-1.500515,0.057224$

$\mathrm{O},-0.636168,-1.821075,-0.008031$

C, $-0.73173,-2.693156,-1.133056$

C, $0.161469,-3.911151,-0.964027$

H, $-0.471032,-2.150304,-2.047233$

H, $-1.779377,-2.987322,-1.200161$

H, $-0.106545,-4.451267,-0.054559$

H, $1.208008,-3.605471,-0.892314$

H, $0.060004,-4.585645,-1.817621$

Cyclic-gauche $(\mathrm{EtOH})_{5}$

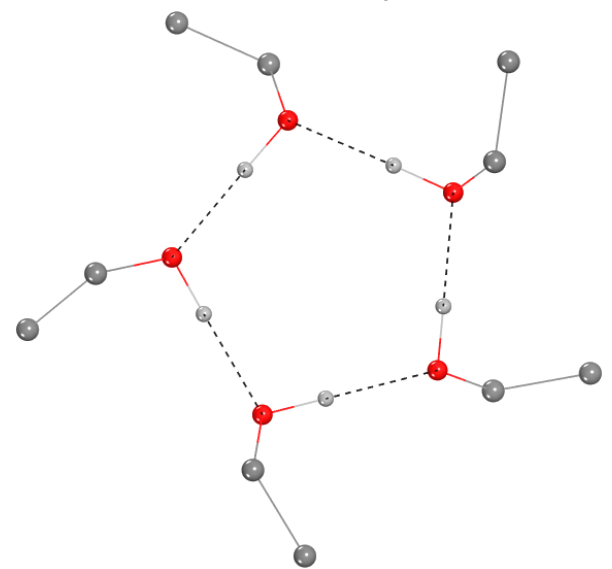

C, 4.401358, 1.007868, - -0.114152

C, 3.399627, 0.136191, 0.626084

$\mathrm{O}, 2.258172,-0.138073,-0.181015$

$\mathrm{H}, 1.756726,0.701256,-0.305339$

H, 4.723198, $0.512836,-1.031664$

H, 3.947763, 1.964364, -0.384239
H, 5.278234, 1.203661, 0.507929

H, 3.839179, $-0.830564,0.874902$

H, 3.08813, 0.612719, 1.561848

$\mathrm{H}, 1.193523,-1.484972,0.107741$

$\mathrm{O}, 0.570391,-2.247434,0.119786$

C, $0.882143,-3.084682,-0.993748$

C, $2.279649,-3.668994,-0.87107$

H, $0.784914,-2.515694,-1.923362$

H, 0.130457, -3.874887, -0.9986

H, 2.372935, -4.230539, 0.060047

H, 3.023368, $-2.868974,-0.874307$

H, 2.492386, -4.33766, -1.708686

H, $-1.038704,-1.609933,0.095811$

$\mathrm{O},-1.946719,-1.228278,0.151944$

C, $-2.556067,-1.707948,1.349378$

C, $-2.726094,-3.217927,1.313997$

H, $-3.523996,-1.210088,1.419133$

H, $-1.957748,-1.409246,2.215765$

H, $-1.750947,-3.705059,1.243541$

H, $-3.325184,-3.508727,0.449356$

H, -3.221896, -3.57183, 2.221198

H, $-1.844972,0.488386,-0.056961$

$\mathrm{O},-1.782031,1.452943,-0.252725$

C, $-2.628858,1.740226,-1.364342$

C, $-4.083193,1.43707,-1.043042$

H, $-2.301715,1.168108,-2.237973$

H, $-2.493045,2.799998,-1.583431$

H, $-4.403846,2.010733,-0.171695$

H, $-4.207322,0.373577,-0.826899$

H, $-4.726239,1.692336,-1.888833$

H, $-0.112787,1.925273,-0.372886$

$\mathrm{O}, 0.83342,2.192849,-0.301053$

C, $0.953994,3.030061,0.847591$

C, $0.137327,4.302747,0.692941$

H, 2.015136, 3.264226, 0.945815

$\mathrm{H}, 0.641276,2.481645,1.742528$

H, $-0.922947,4.059152,0.597975$

H, $0.453892,4.845252,-0.199444$

H, $0.265048,4.950921,1.563416$ 


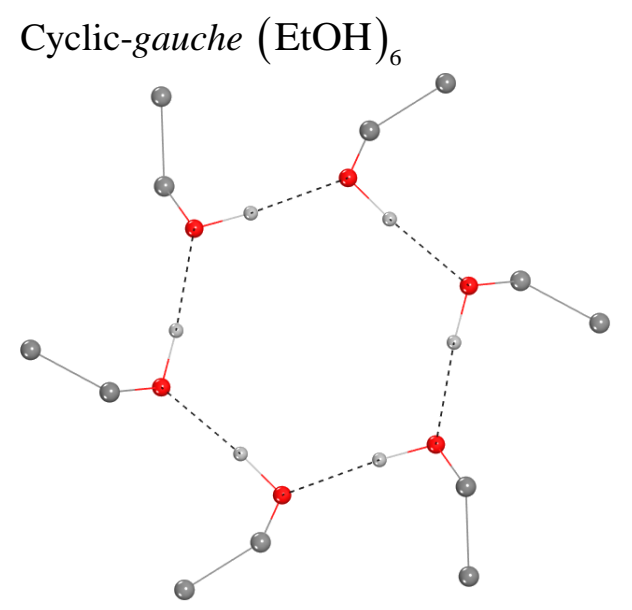

$\mathrm{H},-1.245441,-1.959033,0.034614$

O, $-0.550883,-2.646658,-0.093962$

C, $-0.910431,-3.434519,-1.22751$

C, $-2.18156,-4.226936,-0.969206$

H, $-0.068365,-4.102876,-1.412909$

$\mathrm{H},-1.031189,-2.790112,-2.103505$

$\mathrm{H},-3.018212,-3.548159,-0.790662$

$\mathrm{H},-2.055268,-4.865269,-0.092862$

$\mathrm{H},-2.42586,-4.854952,-1.829554$

$\mathrm{H}, 1.075011,-2.058197,-0.035832$

$\mathrm{O}, 2.018155,-1.801457,0.092168$

C, $2.520068,-2.506455,1.226182$

C, 2.56872, $-4.003664,0.968765$

$\mathrm{H}, 1.902065,-2.28749,2.102175$

$\mathrm{H}, 3.520434,-2.112491,1.411205$

H, 3.184076, -4.214731, 0.092297

$\mathrm{H}, 1.561918,-4.387429,0.790778$

H, 2.990039, -4.529372, 1.8292

H, 2.320457, $-0.099122,0.034463$

H, 5.241396, 0.650261, -0.090605

$\mathrm{H}, 4.581627,-0.840335,-0.791981$

$\mathrm{H}, 5.419052,0.327526,-1.827778$

$\mathrm{H}, 3.589708,1.993421,-1.410259$

$\mathrm{H}, 2.933314,0.504581,-2.102909$

$\mathrm{H}, 1.245383,1.959063,-0.034422$

O, 0.550926, 2.646757, 0.094306

C, $0.910883,3.434645,1.22769$

C, 2.182036, 4.226891, 0.969003

H, 1.0318, 2.790284, 2.103695

H, 0.068952, 4.103118, 1.413267

H, 2.055583, 4.865198, 0.092665

H, 3.018546, 3.547995, 0.790263

H, 2.426656, 4.854916, 1.829256

$\mathrm{H},-1.074899,2.058116,0.036149$

$\mathrm{O},-2.017981,1.80126,-0.092073$

C, $-2.519855,2.506429,-1.225993$

C, $-2.569087,4.003541,-0.968101$

H, $-3.520035,2.112167,-1.411394$

H, -1.901554, 2.287955, -2.101898

$\mathrm{H},-1.56246,4.387587,-0.789704$

$\mathrm{H},-3.184751,4.214115,-0.091731$

$\mathrm{H},-2.990338,4.529399,-1.828476$

$\mathrm{H},-2.320518,0.098944,-0.034518$

O, $-2.5689,-0.846493,0.092854$

C, $-3.431645,-0.930071,1.225733$

C, $-4.752353,-0.223331,0.967668$

H, $-2.933939,-0.505387,2.102743$

$\mathrm{H},-3.590872,-1.993633,1.409333$

H, $-5.241526,-0.64917,0.089741$

$\mathrm{H},-4.581237,0.84084,0.791878$

H, -5.419509, - $0.327031,1.826988$

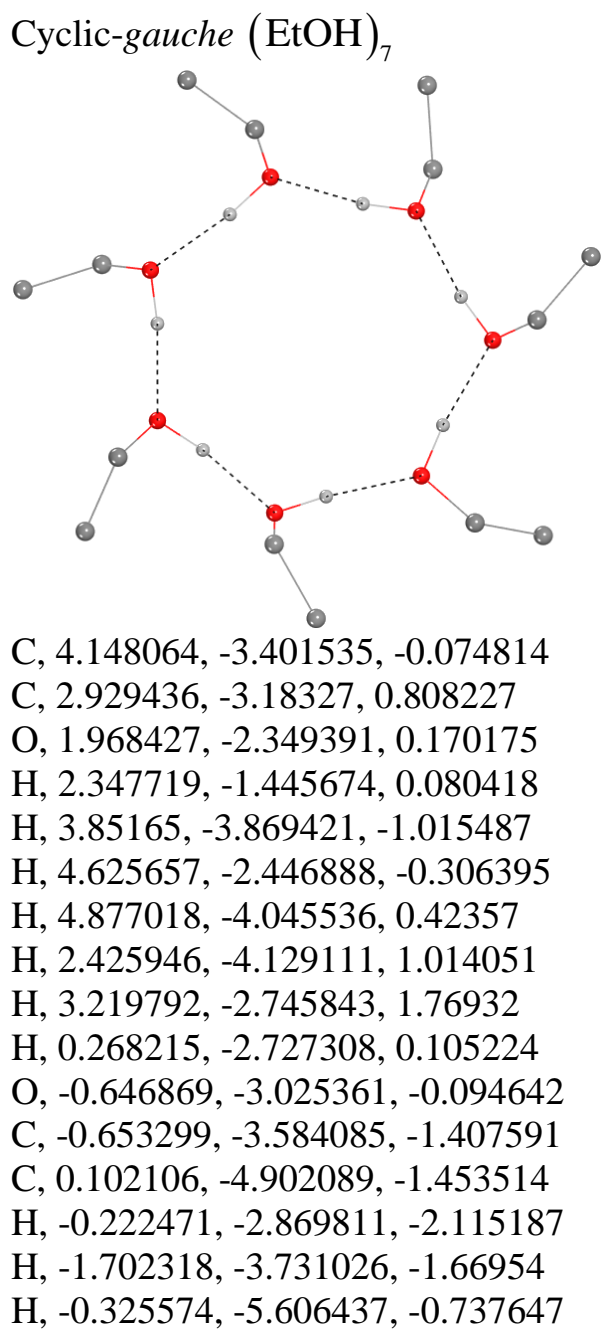


H, 1.152928, -4.742761, -1.202941

$\mathrm{H}, 0.050411,-5.341097,-2.453001$

H, -1.936123, -1.909186, 0.193492

O, $-2.748732,-1.390501,0.396378$

C, $-3.451036,-2.045707,1.449478$

C, $-4.028947,-3.372958,0.985292$

$\mathrm{H},-4.245222,-1.363719,1.757313$

H, $-2.783219,-2.194392,2.303136$

$\mathrm{H},-3.225289,-4.046431,0.67994$

$\mathrm{H},-4.695425,-3.215907,0.134857$

H, $-4.593081,-3.85156,1.789749$

$\mathrm{H},-2.759537,0.338141,0.257133$

$\mathrm{O},-2.888727,1.292064,0.051952$

C, $-3.756238,1.388188,-1.076135$

C, $-5.164637,0.931267,-0.732697$

H, -3.352859, 0.798384, -1.904611

$\mathrm{H},-3.75215,2.437789,-1.374273$

$\mathrm{H},-5.558036,1.517845,0.099595$

$\mathrm{H},-5.158713,-0.122664,-0.446747$

H, -5.829034, 1.050895, -1.592185

$\mathrm{H},-1.475141,2.290926,0.003879$

O, $-0.755925,2.960769,0.069804$

C, $-1.040432,3.809807,1.179686$

C, $-2.255886,4.68212,0.910761$

H, -0.150087, 4.4222, 1.331306

$\mathrm{H},-1.192738,3.205055,2.078513$

$\mathrm{H},-3.140695,4.058826,0.765316$

$\mathrm{H},-2.097761,5.280725,0.011572$

H, $-2.442509,5.354345,1.752026$

$\mathrm{H}, 0.896618,2.493032,-0.162631$

O, $1.849715,2.371748,-0.378356$

C, $2.14376,3.115481,-1.558661$

C, 2.033711, 4.611736, - 1.313622

H, $1.473343,2.808308,-2.366787$

H, 3.16176, 2.843893, -1.842754

H, 2.702043, 4.91075, - 0.503648

H, 1.009994, 4.873355, - 1.037851

H, 2.301594, 5.17055, -2.213834

$\mathrm{H}, 2.658074,0.844824,-0.168417$

$\mathrm{O}, 3.228675,0.07531,0.058279$

C, $4.017032,0.44168,1.188834$

C, 4.974998, 1.573981, 0.85484

$\mathrm{H}, 4.57019,-0.453084,1.479466$

H, 3.364985, 0.723312, 2.021549

$\mathrm{H}, 4.414849,2.467406,0.57213$

$\mathrm{H}, 5.620775,1.285972,0.023251$

H, 5.600113, 1.816527, 1.717902
Cyclic-gauche (EtOH)

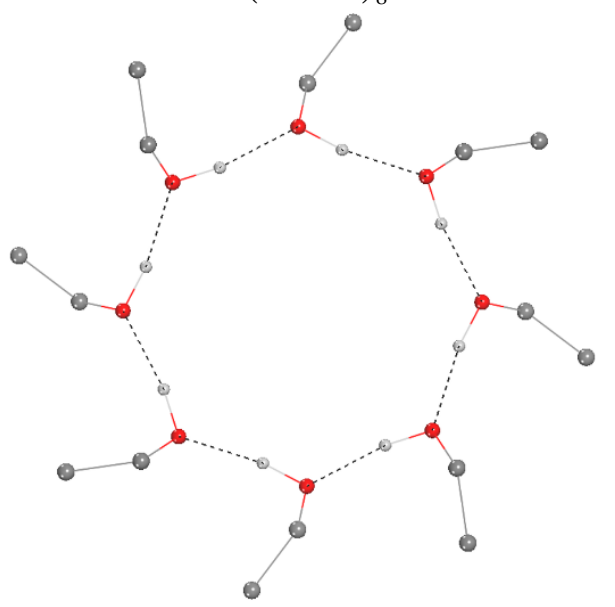

C, $-5.538042,0.990179,-0.976384$

C, $-4.353735,0.09626,-1.30684$

$\mathrm{O},-3.506187,-0.083395,-0.174585$

$\mathrm{H},-3.057854,0.771985,0.014301$

$\mathrm{H},-6.105474,0.57059,-0.143266$

$\mathrm{H},-5.18973,1.986472,-0.69619$

$\mathrm{H},-6.20142,1.08682,-1.839583$

$\mathrm{H},-4.690497,-0.900654,-1.596124$

$\mathrm{H},-3.777417,0.5136,-2.137708$

$\mathrm{H},-2.708411,-1.615111,-0.003908$

$\mathrm{O},-2.41884,-2.536718,0.183635$

C, $-3.151686,-3.016233,1.308571$

C, $-4.617601,-3.227501,0.965979$

$\mathrm{H},-3.049137,-2.31589,2.142621$

H, $-2.682691,-3.958049,1.598348$

$\mathrm{H},-4.712205,-3.922877,0.129534$

$\mathrm{H},-5.078423,-2.278017,0.685676$

$\mathrm{H},-5.159672,-3.634087,1.823519$

$\mathrm{H},-0.771987,-3.057838,0.014467$

O, 0.083394, -3.506179, -0.174394

C, $-0.096259,-4.353796,-1.306597$

C, $-0.990161,-5.538095,-0.976065$

H, 0.900656, -4.690563, -1.595869

H, $-0.513613,-3.777532,-2.137496$

H, $-1.986457,-5.189779,-0.695885$

H, $-0.570559,-6.105471,-0.142915$

$\mathrm{H},-1.0868,-6.201525,-1.839223$

H, 1.615116, -2.7084, -0.003778

O, 2.536729, - $2.418825,0.183725$

C, 3.016262, -3.151597, 1.308701

C, 3.227502, $-4.61754,0.966211$

H, 2.315943, -3.048978, 2.142762

H, 3.958092, -2.682594, 1.59842

H, 3.922855, -4.712216, 0.129755

H, 2.278005, -5.07837, 0.685966

$\mathrm{H}, 3.634102,-5.159554,1.823781$ 
H, 3.057852, $-0.771984,0.014444$

$\mathrm{O}, 3.506188,0.083385,-0.17448$

C, $4.353765,-0.096334,-1.306703$

C, 5.538069, $-0.990227,-0.976164$

$\mathrm{H}, 4.690529,0.900564,-1.596039$

H, 3.777469, $-0.513728,-2.13756$

H, 5.189755, - $1.986506,-0.695918$

$\mathrm{H}, 6.105478,-0.570585,-0.143057$

H, 6.201468, -1.086917, -1.839341

$\mathrm{H}, 2.70841,1.615111,-0.003909$

$\mathrm{O}, 2.418835,2.53673,0.183572$

C, 3.151651, 3.016309, 1.3085

C, 4.617575, 3.227559, 0.965935

H, 3.04908, 2.316014, 2.142588

$\mathrm{H}, 2.682648,3.958142,1.598211$

$\mathrm{H}, 4.712201,3.922886,0.129452$

H, 5.078404, 2.278059, 0.6857

H, 5.159623, 3.634195, 1.823466

H, 0.771988, 3.05784, 0.01432

O, $-0.083387,3.506167,-0.1746$

C, $0.096306,4.353715,-1.306848$

C, $0.990187,5.53804,-0.976354$

H, - $0.900601,4.690457,-1.596181$

H, 0.513696, 3.777401, - 2.137695

$\mathrm{H}, 1.986474,5.189748,-0.696112$

$\mathrm{H}, 0.570548,6.105466,-0.143257$

$\mathrm{H}, 1.086856,6.201417,-1.83955$

H, -1.615114, 2.708397, -0.003989

$\mathrm{O},-2.536734,2.418834,0.183502$

C, $-3.0163,3.15167,1.308423$

C, $-3.227529,4.617594,0.965843$

H, $-2.316004,3.049098,2.14251$

$\mathrm{H},-3.958138,2.682684,1.598142$

$\mathrm{H},-3.922859,4.712222,0.129362$

$\mathrm{H},-2.278024,5.078407,0.685599$

H, -3.634153, 5.159656, 1.82337

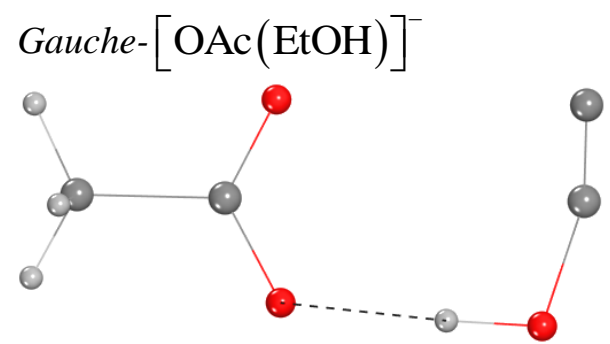

O, -0.97246, 1.057486, -0.582029

$\mathrm{O},-0.93039,-1.027213,0.280610$

C, $-1.50133,0.047307,-0.071155$

C, $-3.02124,0.082319,0.181641$
$\mathrm{H},-3.45644,1.014167,-0.180172$

H, $-3.49042,-0.770276,-0.315333$

$\mathrm{H},-3.20755,-0.023053,1.253633$

C, 2.184226, 0.01289, -0.497571

$\mathrm{H}, 1.525691,0.407987,-1.277212$

H, 3.191198, -0.107108, - 0.913318

C, 2.210626, 1.000879, 0.664122

H, 2.597147, 1.974776, 0.342379

H, 1.192753, 1.140551, 1.030894

H, 2.839299, 0.615468, 1.472135

$\mathrm{O}, 1.7534,-1.267983,-0.089247$

H, 0.770253, -1.211203, 0.050098

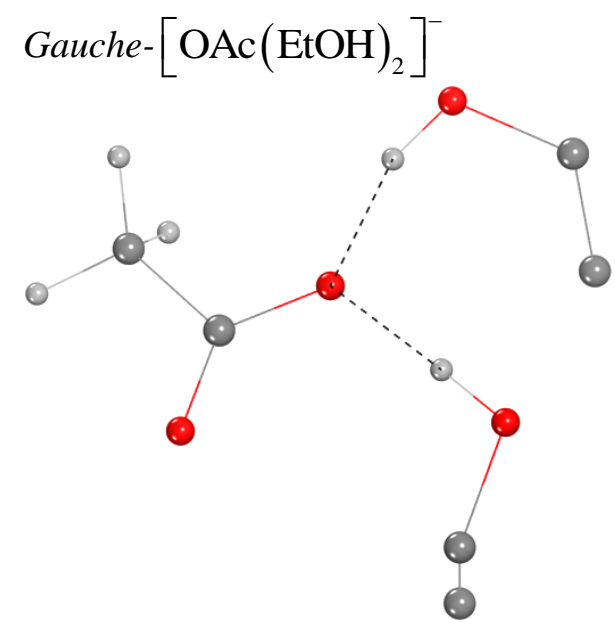

$\mathrm{O},-0.16671,0.803228,-0.449171$

$\mathrm{O}, 1.733615,1.877949,0.083347$

C, $0.499797,1.81185,-0.033266$

C, $-0.35132,3.029432,0.347433$

$\mathrm{H},-0.90646,3.36827,-0.530723$

$\mathrm{H},-1.08625,2.738706,1.102073$

$\mathrm{H}, 0.275549,3.836512,0.725022$

O, 1.194644, -1.475124, -1.121979

C, $2.431567,-1.376365,-0.44499$

H, 3.048102, $-2.210924,-0.794184$

H, 2.933053, $-0.437977,-0.701006$

C, 2.257934, $-1.44765,1.068325$

$\mathrm{H}, 3.22856,-1.42407,1.575853$

$\mathrm{H}, 1.675331,-0.587751,1.403118$

$\mathrm{H}, 1.732124,-2.365763,1.344736$

$\mathrm{H}, 0.701371,-0.637376,-0.939609$

O, $-2.79458,0.193571,-0.316514$

C, $-2.57493,-1.203897,-0.34249$

$\mathrm{H},-3.54288,-1.673336,-0.542927$

$\mathrm{H},-1.89321,-1.468131,-1.158741$

C, $-2.0024,-1.71641,0.974304$

$\mathrm{H},-1.03595,-1.244226,1.157567$

H, -2.67896, -1.471964, 1.797732 
$\mathrm{H},-1.85462,-2.800722,0.941021$

H, $-1.89537,0.599992,-0.361286$

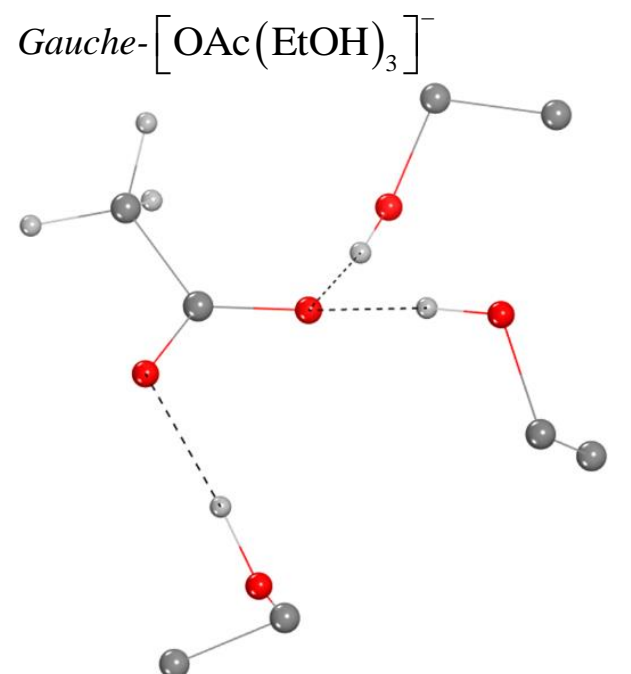

$\mathrm{O}, 0.278074,-0.634567,-0.259853$

$\mathrm{O},-1.495982,-1.666261,-1.168704$

C, $-0.340796,-1.658919,-0.695724$

C, $0.427403,-2.979156,-0.646707$

H, 1.163015, -2.976823, -1.456099

H, 0.97294, -3.065752, 0.294294

H, $-0.248789,-3.82188,-0.784045$

$\mathrm{O}, 0.647684,1.803245,-1.514662$

C, $-0.210399,2.636735,-0.754249$

$\mathrm{H},-0.238971,3.603673,-1.264525$

H, $-1.224813,2.227064,-0.746276$

C, 0.30099, 2.81346, 0.6722

H, $-0.373226,3.450632,1.254591$

H, 0.373492, 1.840963, 1.164086

H, 1.296567, 3.264887, 0.664799

O, 2.394328, -0.944194, 1.428835

C, 3.476574, $-0.779156,0.536327$

$\mathrm{H}, 4.358091,-1.20094,1.027952$

H, 3.302075, $-1.35288,-0.383493$

C, 3.718519, 0.68518, 0.187901

$\mathrm{H}, 2.852016,1.105473,-0.326382$

$\mathrm{H}, 3.889186,1.258293,1.102933$

$\mathrm{H}, 4.593421,0.791384,-0.462826$

$\mathrm{H}, 1.57067,-0.806213,0.902837$

$\mathrm{H}, 0.50016,0.888865,-1.191337$

$\mathrm{O},-3.087108,0.500657,-0.544968$

C, $-2.813971,0.488446,0.843017$

$\mathrm{H},-1.737819,0.381545,1.012413$

$\mathrm{H},-3.127065,1.459629,1.238944$

C, $-3.563901,-0.633339,1.554977$
$\mathrm{H},-3.247944,-1.596968,1.150215$

H, -3.36208, $-0.620386,2.631272$

H, $-4.639786,-0.527048,1.394281$

$\mathrm{H},-2.533628,-0.214072,-0.935268$

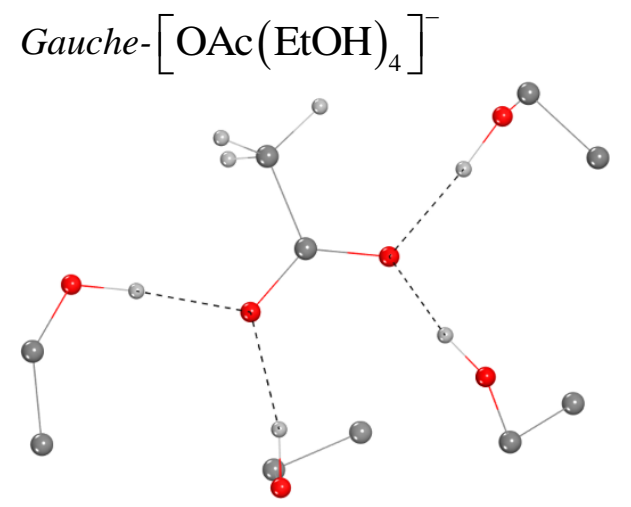

$\mathrm{O}, 0.981734,-0.563675,0.081442$

$\mathrm{O},-1.231533,-0.614423,-0.230790$

C, $-0.144075,-1.128092,0.150548$

C, $-0.217468,-2.531321,0.740502$

$\mathrm{H},-0.640882,-3.207303,-0.005305$

$\mathrm{H}, 0.76509,-2.881472,1.053153$

H, $-0.901334,-2.527727,1.591983$

$\mathrm{O}, 1.8835,1.290152,-1.79192$

C, $1.735258,2.505331,-1.077287$

$\mathrm{H}, 1.977183,3.308553,-1.778204$

$\mathrm{H}, 0.695449,2.636366,-0.761985$

C, 2.665382, 2.564813, 0.129881

$\mathrm{H}, 2.547105,3.510082,0.670056$

$\mathrm{H}, 2.442215,1.74317,0.813963$

$\mathrm{H}, 3.705965,2.468463,-0.190077$

O, 3.227696, -1.689049, 1.202898

C, 3.895133, -2.118415, 0.032301

$\mathrm{H}, 4.630285,-2.863869,0.347473$

H, 3.189253, $-2.612115,-0.647925$

C, $4.586555,-0.968681,-0.690751$

$\mathrm{H}, 3.855452,-0.232962,-1.03164$

H, 5.285903, $-0.474032,-0.011915$

H, 5.140182, $-1.33422,-1.562166$

H, 2.416759, $-1.217557,0.909116$

$\mathrm{H}, 1.486697,0.588989,-1.234917$

$\mathrm{O},-1.85682,2.061418,-0.202851$

C, $-2.051325,2.06402,1.19907$

H, -2.692141, 2.920609, 1.424897

$\mathrm{H},-2.581442,1.155309,1.5083$

C, $-0.731962,2.175835,1.954534$

H, $-0.221562,3.103836,1.685176$

H, $-0.897509,2.166487,3.036887$ 
$\mathrm{H},-0.07826,1.341479,1.691555$

H, -1.501126, 1.174106, -0.425049

O, -3.613346, -1.937153, 0.097091

H, $-2.736834,-1.491226,0.053365$

C, $-4.391635,-1.428345,-0.967704$

$\mathrm{H},-5.257038,-2.089496,-1.065638$

$\mathrm{H},-3.828426,-1.475074,-1.908039$

C, $-4.850934,0.003206,-0.714797$

$\mathrm{H},-3.993992,0.676409,-0.64515$

$\mathrm{H},-5.497598,0.353275,-1.526433$

H, $-5.408805,0.051651,0.223789$

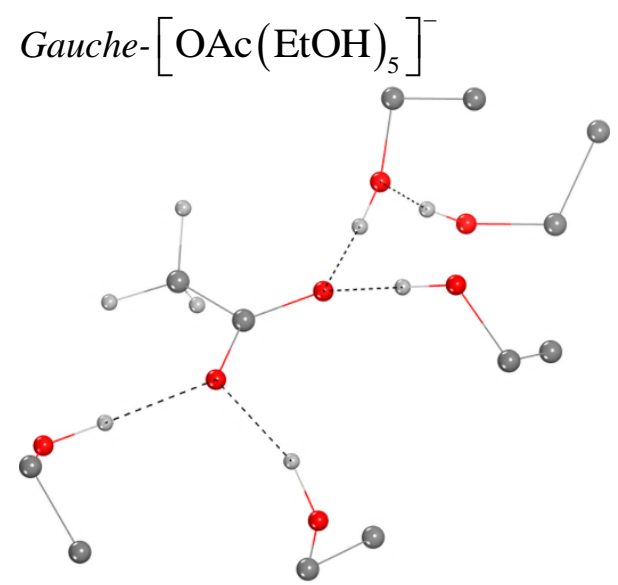

$\mathrm{O}, 1.55106,-0.50686,-0.162633$

O, $-0.56019,-0.17881,-0.80966$

C, 0.400414, - $-0.92293,-0.457815$

C, $0.152241,-2.42488,-0.419281$

H, $0.351468,-2.8226,-1.418426$

$\mathrm{H}, 0.829362,-2.90754,0.284754$

$\mathrm{H},-0.886537,-2.64541,-0.173208$

$\mathrm{O}, 3.080216,1.4883,-1.382507$

C, 2.967192, 2.555263, -0.455034

H, 3.474542, 3.411368, -0.906915

$\mathrm{H}, 1.915806,2.823066,-0.312018$

C, 3.612516, 2.207909, 0.882084

H, 3.529995, 3.042819, 1.585886

$\mathrm{H}, 3.121524,1.335703,1.319193$

$\mathrm{H}, 4.669337,1.968541,0.740473$

O, 3.366147, -2.18757, 1.061871

C, 4.170904, $-2.52642,-0.051072$

$\mathrm{H}, 4.709021,-3.43868,0.219375$

H, 3.540007, $-2.75604,-0.919542$

C, $5.154798,-1.41868,-0.40778$

H, 4.62347, $-0.51028,-0.698705$

H, 5.782495, -1.19064, 0.457285

$\mathrm{H}, 5.798596,-1.72361,-1.239547$

$\mathrm{H}, 2.708938,-1.53135,0.744244$
$\mathrm{H}, 2.483957,0.779807,-1.067879$

O, $-0.729032,2.542654,-0.34361$

C, $-1.222889,2.388457,0.973607$

H, -1.692764, 3.337719, 1.243434

$\mathrm{H},-1.99759,1.612597,0.99952$

C, $-0.116326,2.043244,1.962849$

$\mathrm{H}, 0.633111,2.838108,1.98243$

H, $-0.52019,1.914819,2.972351$

$\mathrm{H}, 0.380239,1.11801,1.662663$

$\mathrm{H},-0.48703,1.643918,-0.652998$

O, $-3.006881,-1.08248,-1.393994$

H, $-2.087325,-0.79402,-1.155712$

C, $-3.509937,-0.1836,-2.371808$

$\mathrm{H},-4.404801,-0.65037,-2.789005$

H, -2.778044, -0.07429, -3.179144

C, $-3.845646,1.178349,-1.779283$

$\mathrm{H},-2.949412,1.65465,-1.376083$

$\mathrm{H},-4.267937,1.836754,-2.544995$

$\mathrm{H},-4.578851,1.063579,-0.977052$

$\mathrm{O},-4.625127,-1.54767,0.833242$

$\mathrm{H},-4.074723,-1.45266,0.028062$

C, $-4.125105,-0.65139,1.807467$

$\mathrm{H},-4.808931,-0.7119,2.657119$

$\mathrm{H},-4.153818,0.380303,1.433255$

C, $-2.708026,-0.99832,2.24751$

$\mathrm{H},-2.670832,-2.02874,2.607908$

$\mathrm{H},-2.009218,-0.89475,1.414601$

$\mathrm{H},-2.37423,-0.33146,3.04834$

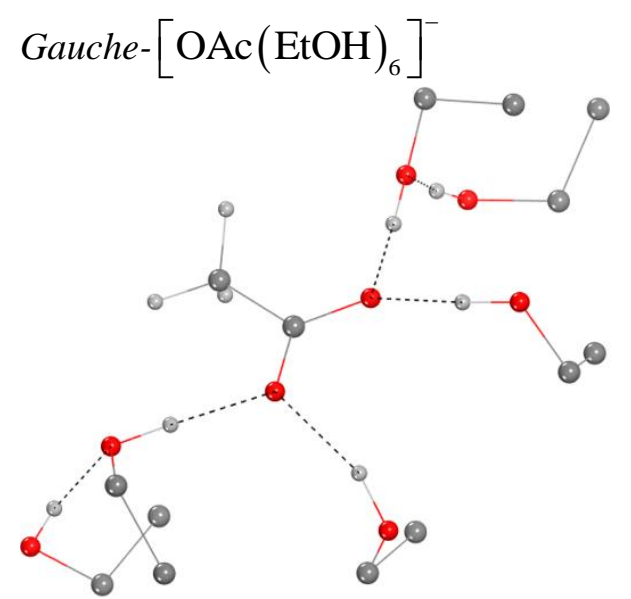

$\mathrm{O}, 0.688385,0.279087,-0.862820$

$\mathrm{O},-1.53895,0.389478,-0.896008$

C, $-0.45003,-0.215344,-1.092822$

C, $-0.49683,-1.646353,-1.609002$

$\mathrm{H}, 0.219687,-1.767473,-2.422699$

H, $-0.18821,-2.317698,-0.803804$ 
H, -1.49818, -1.918237, -1.940153

O, 1.587625, 2.922085, - 0.89474

C, 1.625528, 3.247786, 0.485321

$\mathrm{H}, 1.941292,4.292114,0.546172$

$\mathrm{H}, 0.623664,3.172357,0.918913$

C, 2.602175, 2.359077, 1.248707

H, 2.628907, 2.627437, 2.309876

H, 2.301106, 1.312414, 1.164561

$\mathrm{H}, 3.608496,2.460079,0.834323$

$\mathrm{O}, 2.925442,-1.212133,-0.962068$

C, 3.580551, $-0.761104,-2.137352$

H, 4.362108, -1.493324, -2.354608

$\mathrm{H}, 2.875284,-0.76721,-2.976568$

C, 4.17644, 0.629304, -1.966865

$\mathrm{H}, 3.398125,1.362718,-1.748111$

$\mathrm{H}, 4.891337,0.633398,-1.140292$

$\mathrm{H}, 4.697779,0.93631,-2.879134$

$\mathrm{H}, 2.092782,-0.687257,-0.851607$

$\mathrm{H}, 1.157304,2.046717,-0.967097$

O, $-1.90365,2.376022,1.006928$

C, $-2.08046,1.441614,2.055882$

H, $-2.59128,1.973264,2.862508$

$\mathrm{H},-2.7329,0.623531,1.728299$

C, $-0.75429,0.876919,2.550132$

$\mathrm{H},-0.11782,1.68173,2.925354$

$\mathrm{H},-0.91327,0.150601,3.353381$

H, $-0.22619,0.382636,1.731843$

$\mathrm{H},-1.63942,1.859266,0.217971$

$\mathrm{O},-3.95886,-0.581078,-1.53265$

H, $-3.04907,-0.271199,-1.292995$

C, $-4.75887,0.562401,-1.800548$

$\mathrm{H},-5.66797,0.195437,-2.281316$

$\mathrm{H},-4.23956,1.213973,-2.511083$

C, $-5.09944,1.331869,-0.531664$

H, $-4.19339,1.716199,-0.058324$

$\mathrm{H},-5.75162,2.180474,-0.760604$

$\mathrm{H},-5.61708,0.675631,0.172369$

O, $-5.02777,-2.440129,0.261093$

H, $-4.66898,-1.84055,-0.425266$

C, $-4.40143,-2.113734,1.487388$

H, $-4.86451,-2.74921,2.245338$

$\mathrm{H},-4.60765,-1.070382,1.760006$

C, $-2.89602,-2.347213,1.452499$

$\mathrm{H},-2.6847,-3.381568,1.172403$

H, -2.42227, $-1.686106,0.723666$

H, -2.44672, $-2.146465,2.429749$

O, 4.26862, - $2.42835,1.140368$

H, 3.724871, -2.139842, 0.381427

C, 5.0867, $-1.333619,1.504905$

$\mathrm{H}, 4.556117,-0.390444,1.335365$

H, 5.281959, -1.418759, 2.576973
C, 6.405086, -1.339918, 0.738718

H, 6.218813, -1.262922, -0.334956

H, 7.041848, $-0.502579,1.041104$

H, 6.937915, -2.27503, 0.924401

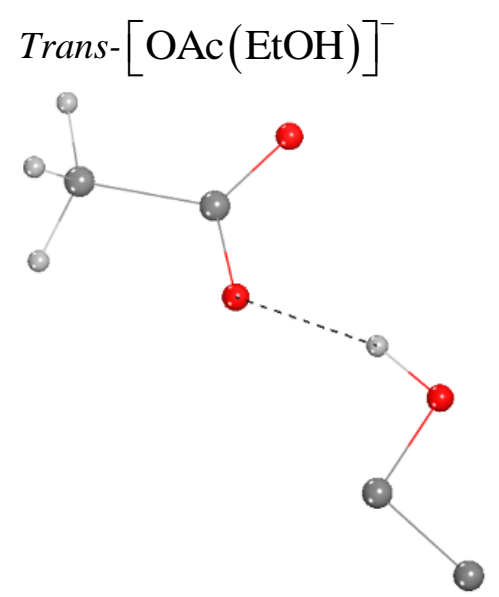

O, $-2.331578,1.318065,-0.027571$

$\mathrm{O},-0.809361,-0.349408,0.030768$

C, $-1.987396,0.121361,-0.000489$

C, $-3.099502,-0.94846,-0.003164$

H, $-4.086409,-0.486219,-0.038345$

$\mathrm{H},-2.963858,-1.607269,-0.864743$

$\mathrm{H},-3.007858,-1.564556,0.895113$

C, 2.250327, $-0.436449,-0.019605$

H, 1.966558, $-0.987979,-0.925801$

H, 1.97128, -1.064657, 0.83665

C, 3.751618, $-0.190185,-0.013536$

H, 4.308765, $-1.131445,-0.057932$

$\mathrm{H}, 4.030226,0.42612,-0.871663$

$\mathrm{H}, 4.036443,0.346729,0.894431$

$\mathrm{O}, 1.595288,0.805232,0.036272$

$\mathrm{H}, 0.61978,0.600571,0.037298$ 


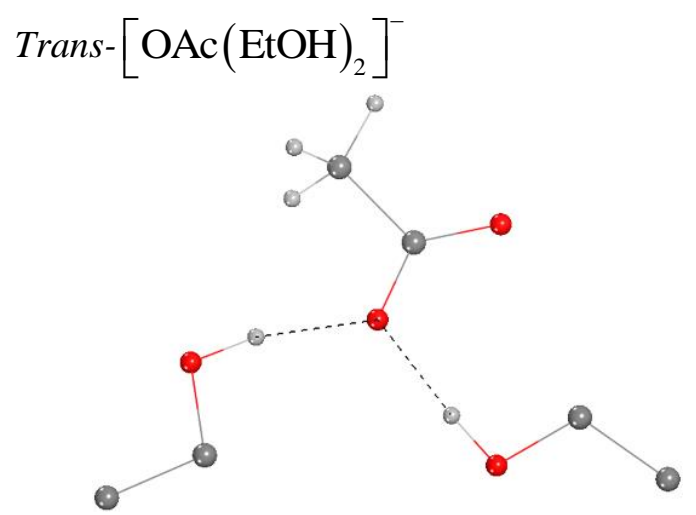

$\mathrm{O},-0.07984,0.759503,-0.059824$

$\mathrm{O}, 1.701377,2.132781,-0.051373$

C, $0.481275,1.908677,-0.037072$

C, $-0.492207,3.09279,0.016465$

$\mathrm{H},-1.155711,3.056159,-0.850992$

$\mathrm{H},-1.12057,3.005639,0.906324$

$\mathrm{H}, 0.049742,4.037731,0.033011$

O, 1.462071, - $1.484264,-0.233837$

C, 2.639391, $-1.138793,0.468487$

H, 2.780005, $-0.054987,0.446226$

H, 2.55877, - $1.449638,1.521009$

C, $3.824059,-1.846308,-0.173333$

H, 4.749439, -1.641496, 0.374601

H, 3.654776, -2.926413, -0.185389

H, 3.941943, $-1.508151,-1.204972$

$\mathrm{H}, 0.874369,-0.689421,-0.219839$

O, $-2.704034,0.18903,0.040547$

C, $-2.579002,-1.215407,-0.027483$

$\mathrm{H},-2.073257,-1.514669,-0.953731$

H, $-1.971596,-1.590763,0.804992$

C, $-3.970429,-1.824137,0.025704$

H, $-4.468754,-1.534673,0.953872$

$\mathrm{H},-4.570289,-1.456511,-0.810124$

$\mathrm{H},-3.924225,-2.915804,-0.025357$

$\mathrm{H},-1.779754,0.545664,0.009644$

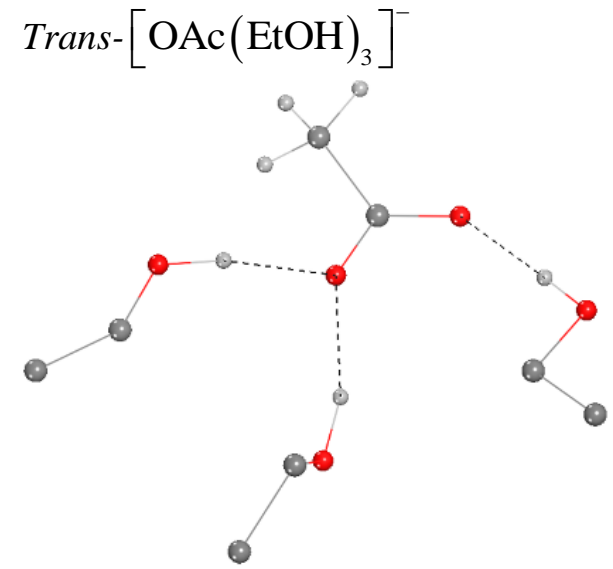

$\mathrm{O}, 0.02658,1.058072,0.059403$

$\mathrm{O}, 1.790831,2.301438,-0.564457$

C, $0.612485,2.162589,-0.182515$

C, $-0.237366,3.417543,0.02259$

$\mathrm{H},-1.105765,3.375631,-0.639464$

$\mathrm{H},-0.615442,3.438159,1.047033$

$\mathrm{H}, 0.344811,4.314653,-0.181927$

$\mathrm{O},-0.281816,-1.374764,-1.130613$

C, $-0.568424,-2.09631,0.051212$

H, $0.360849,-2.435247,0.529845$

$\mathrm{H},-1.097911,-1.460167,0.768551$

C, $-1.422707,-3.297309,-0.318974$

$\mathrm{H},-1.649478,-3.903715,0.562518$

H, $-2.360905,-2.962777,-0.767877$

H, $-0.897806,-3.917923,-1.048975$

$\mathrm{O},-2.496505,0.844449,1.012862$

C, $-3.181539,0.442764,-0.157227$

$\mathrm{H},-3.523182,1.321504,-0.723258$

$\mathrm{H},-2.517622,-0.133849,-0.809119$

C, $-4.381653,-0.398223,0.247488$

H, $-4.045858,-1.291711,0.778798$

$\mathrm{H},-5.029917,0.172417,0.916914$

$\mathrm{H},-4.960676,-0.704516,-0.628674$

$\mathrm{H},-1.554597,1.00471,0.758901$

H, 0.001656, - $0.474549,-0.855714$

$\mathrm{O}, 3.714235,0.350193,-0.103288$

C, 2.968707, $-0.806944,0.222974$

H, 2.229347, $-0.580597,0.999428$

H, 2.417257, -1.174677, -0.649673

C, 3.93692, $-1.871066,0.711256$

$\mathrm{H}, 4.474899,-1.51347,1.592433$

$\mathrm{H}, 3.405997,-2.792083,0.969347$

$\mathrm{H}, 4.669891,-2.094639,-0.067629$

H, 3.069319, 1.059481, -0.323518 


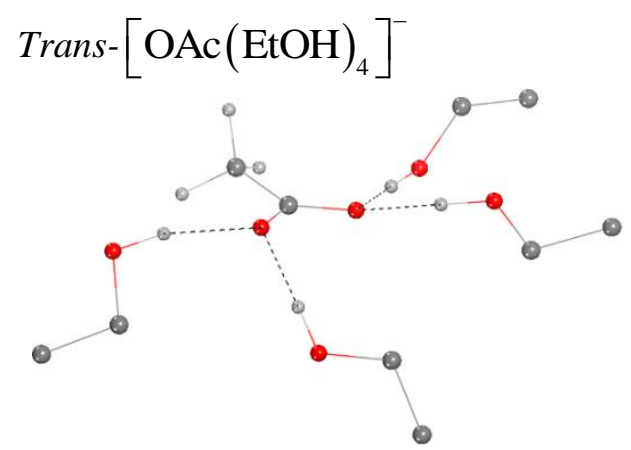

O, $-0.551992,-0.709413,0.027397$

O, 1.584526, - $0.649741,0.68994$

C, $0.575837,-1.241358,0.223522$

C, $0.725008,-2.725001,-0.097019$

$\mathrm{H}, 0.617867,-3.285599,0.835571$

$\mathrm{H},-0.046279,-3.055802,-0.791878$

H, 1.719531, - $2.927491,-0.49577$

$\mathrm{O},-2.224028,1.145955,1.180779$

C, $-2.850448,1.586245,-0.009277$

$\mathrm{H},-2.286134,2.416325,-0.455848$

$\mathrm{H},-2.887998,0.775729,-0.744724$

C, $-4.255574,2.04953,0.336453$

$\mathrm{H},-4.772383,2.426791,-0.550399$

$\mathrm{H},-4.830234,1.219025,0.752794$

$\mathrm{H},-4.212837,2.844074,1.084923$

$\mathrm{O}, 4.160011,-1.56521,0.482314$

H, 3.212197, -1.369159, 0.652247

C, 4.718604, $-0.388676,-0.073835$

$\mathrm{H}, 4.189071,-0.106869,-0.991063$

$\mathrm{H}, 4.622783,0.45148,0.623198$

C, 6.182878, $-0.656385,-0.374057$

H, 6.274524, -1.486725, -1.07791

$\mathrm{H}, 6.66044,0.22742,-0.805598$

$\mathrm{H}, 6.708381,-0.929011,0.543917$

$\mathrm{O},-2.763778,-1.882146,-1.05674$

C, $-3.600311,-1.958277,0.083109$

$\mathrm{H},-3.419378,-2.8947,0.628796$

H, $-3.389773,-1.129544,0.766399$

C, $-5.047684,-1.906792,-0.377312$

H, $-5.240411,-0.962241,-0.891035$

H, $-5.247253,-2.721982,-1.076803$

$\mathrm{H},-5.73264,-1.993928,0.470985$

H, $-1.898696,-1.517362,-0.761218$

H, -1.472786, 0.571427, 0.92239

O, 2.006624, 2.046847, 0.118967

C, $0.731812,2.565148,-0.215613$

H, $0.24565,1.929644,-0.964459$

$\mathrm{H}, 0.079079,2.587919,0.664134$

C, 0.921981, 3.969703, -0.760734

H, 1.559955, 3.945186, -1.647109
$\mathrm{H},-0.039744,4.4165,-1.02851$

H, 1.405123, 4.599372, - 0.010096

$\mathrm{H}, 1.87842,1.114366,0.394402$

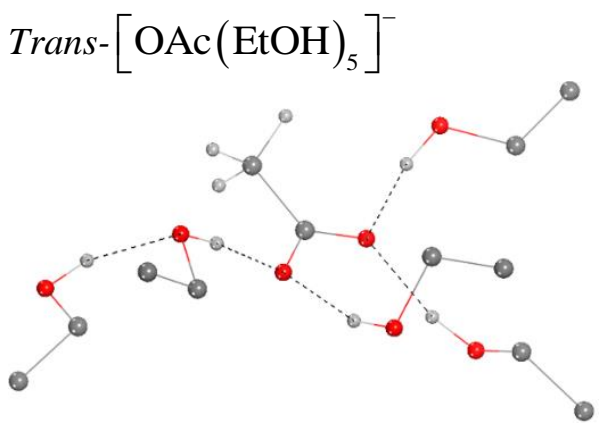

O, $-0.859244,-0.196475,0.077606$

O, 1.165004, -1.145316, 0.12398

C, $-0.037134,-1.102472,-0.241781$

C, $-0.545023,-2.244076,-1.114531$

H, $-0.796714,-3.084148,-0.461846$

$\mathrm{H},-1.43812,-1.953253,-1.667461$

H, 0.238006, -2.574133, -1.797815

$\mathrm{O}, 0.113501,2.366841,0.318983$

C, $0.847978,2.308403,-0.890318$

$\mathrm{H}, 1.517826,1.441584,-0.886169$

$\mathrm{H}, 0.168661,2.197464,-1.746383$

C, $1.646614,3.592784,-1.023685$

$\mathrm{H}, 2.214772,3.603984,-1.957686$

H, $0.975185,4.45433,-1.008431$

$\mathrm{H}, 2.34254,3.688011,-0.187027$

$\mathrm{O},-4.98909,-1.167704,0.089888$

$\mathrm{H},-4.423277,-0.667311,-0.533417$

C, $-4.139355,-1.639997,1.123192$

$\mathrm{H},-3.464972,-2.416216,0.741144$

$\mathrm{H},-3.512992,-0.827763,1.507306$

C, $-5.009495,-2.207341,2.22949$

$\mathrm{H},-5.632194,-3.014681,1.837951$

$\mathrm{H},-4.395534,-2.598255,3.04462$

$\mathrm{H},-5.6668,-1.429476,2.623499$

O, $-3.112917,0.393781,-1.225815$

C, $-3.374288,1.699924,-0.72319$

$\mathrm{H},-3.561921,1.654638,0.356226$

$\mathrm{H},-2.501744,2.340081,-0.880916$

C, $-4.591584,2.250486,-1.442457$

$\mathrm{H},-4.404599,2.290345,-2.517551$

$\mathrm{H},-5.455614,1.606343,-1.264626$

H, $-4.826644,3.256962,-1.087042$

H, -2.273847, 0.082348, -0.797494

$\mathrm{H},-0.251119,1.467556,0.461853$

O, 2.844112, $-0.161979,2.129935$ 
C, 3.119363, 1.159467, 1.702619

H, 3.609099, 1.150219, 0.7182

H, 2.194317, 1.73542, 1.607996

C, 4.041205, 1.802453, 2.723764

$\mathrm{H}, 4.963278,1.223651,2.815066$

$\mathrm{H}, 4.293336,2.825421,2.430728$

$\mathrm{H}, 3.55425,1.825886,3.70078$

$\mathrm{H}, 2.167841,-0.531504,1.527452$

O, 3.173651, $-2.495309,-1.17858$

$\mathrm{H}, 2.380853,-2.154035,-0.7117$

C, $4.191814,-1.527103,-1.005786$

$\mathrm{H}, 3.9082,-0.582711,-1.489314$

$\mathrm{H}, 4.346504,-1.319581,0.058496$

C, 5.467234, -2.06419, -1.630772

H, 5.306419, $-2.270223,-2.691328$

$\mathrm{H}, 6.284514,-1.344802,-1.530345$

H, 5.756385, - $2.996889,-1.142026$
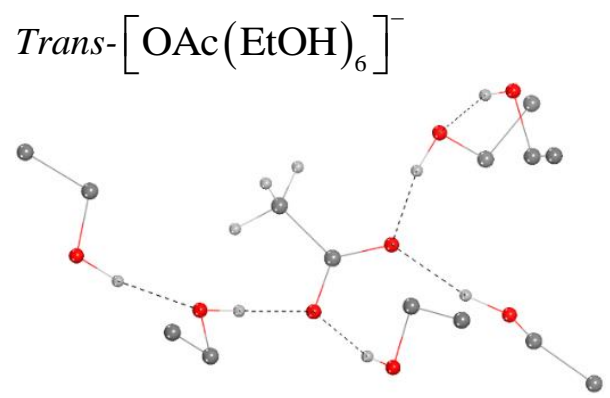

O, 1.007633, 0.412457, -1.35905

O, $-0.857795,-0.713315,-0.863506$

C, 0.392549, $-0.628451,-0.995071$

C, $1.238188,-1.848517,-0.656443$

H, 2.023721, $-1.978275,-1.402323$

H, 1.725719, -1.669769, 0.305517

H, $0.626932,-2.747033,-0.584946$

$\mathrm{O}, 0.126616,2.844015,-0.419147$

C, $-0.173275,2.379262,0.88513$

$\mathrm{H},-0.852754,1.521654,0.837435$

$\mathrm{H}, 0.742138,2.045754,1.393238$

C, $-0.813939,3.515705,1.660714$

$\mathrm{H},-1.042738,3.207948,2.684179$

H, $-0.140459,4.375224,1.690652$

H, -1.740397, 3.824626, 1.170994

O, 5.708127, -1.113369, -0.697087

H, 4.977801, $-0.482012,-0.857615$

C, 5.462486, -1.723818, 0.554913

H, 5.35795, $-0.963722,1.338639$

$\mathrm{H}, 4.528573,-2.298387,0.527599$

C, 6.628065, -2.64374, 0.870332
$\mathrm{H}, 7.556112,-2.069726,0.91061$

$\mathrm{H}, 6.480603,-3.146205,1.829778$

$\mathrm{H}, 6.72798,-3.399255,0.088514$

O, 3.576809, 0.643153, - 0.670224

C, 3.758963, 2.037507, -0.461569

$\mathrm{H}, 4.106051,2.510004,-1.38783$

$\mathrm{H}, 2.804792,2.496556,-0.1895$

C, 4.786654, 2.231899, 0.639876

$\mathrm{H}, 4.421274,1.796604,1.572443$

H, 5.722897, 1.73579, 0.372772

$\mathrm{H}, 4.985752,3.294604,0.801331$

$\mathrm{H}, 2.656861,0.488787,-1.005961$

$\mathrm{H}, 0.378485,2.055561,-0.944685$

O, $-3.107801,0.689863,-1.740836$

C, $-3.185831,1.955781,-1.109003$

$\mathrm{H},-3.353198,1.835815,-0.029562$

$\mathrm{H},-2.253577,2.512627,-1.238947$

C, $-4.349666,2.716092,-1.719428$

$\mathrm{H},-5.275495,2.150858,-1.589948$

H, -4.464865, 3.694985, -1.246171

H, -4.182034, 2.858287, -2.788895

$\mathrm{H},-2.256095,0.271243,-1.500862$

O, -2.315286, -2.850506, -0.08439

$\mathrm{H},-1.684683,-2.135061,-0.341828$

C, $-3.413379,-2.794194,-0.992142$

$\mathrm{H},-3.750313,-1.760523,-1.109453$

$\mathrm{H},-3.09594,-3.158126,-1.975835$

C, $-4.52861,-3.66615,-0.444201$

$\mathrm{H},-4.859634,-3.290512,0.526456$

H, $-5.381619,-3.672429,-1.127571$

H, $-4.174766,-4.69133,-0.313529$

O, $-3.189765,-1.743094,2.3362$

H, $-2.864918,-2.309481,1.609012$

C, $-3.328783,-0.435057,1.80014$

$\mathrm{H},-2.500429,-0.214731,1.120822$

$\mathrm{H},-4.258079,-0.354686,1.220229$

C, $-3.345826,0.555828,2.949907$

$\mathrm{H},-2.404379,0.502898,3.500879$

$\mathrm{H},-3.480909,1.576188,2.581303$

$\mathrm{H},-4.160236,0.318725,3.638447$ 


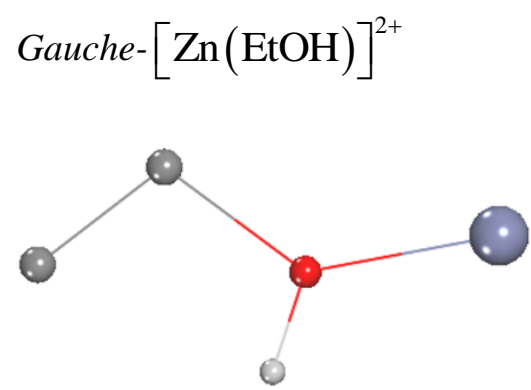

Zn, 1.443589, 0.012616, 0.013369 O, $-0.359069,-0.325028,-0.053052$ $\mathrm{H},-0.664901,-1.25095,-0.158835$ C, $-1.672298,0.648143,-0.03387$ $\mathrm{H},-1.487787,1.249974,0.849285$ H, $-1.564234,1.189919,-0.967098$ C, $-2.851657,-0.256668,0.04934$ $\mathrm{H},-2.881234,-0.833392,0.974497$ $\mathrm{H},-3.728137,0.408069,0.064553$ H, $-2.965088,-0.890732,-0.831874$

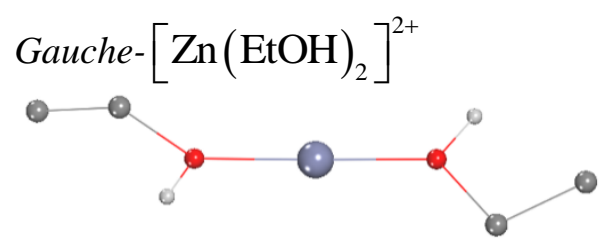

Zn, $-0.002484,-0.249111,-0.04862$ $\mathrm{O},-1.826217,-0.300461,0.216342$ $\mathrm{O}, 1.817689,-0.18466,-0.335897$ H, 2.201837, -0.500494, -1.176428 H, $-2.247491,-1.065198,0.653914$ C, 2.980436, 0.199081, 0.634172 $\mathrm{H}, 2.627704,1.113104,1.101391$ H, 3.02823, $-0.631044,1.331583$ C, 4.218126, 0.379949, -0.191786 H, 4.115306, 1.190156, - 0.914198 H, 5.028127, 0.651189, 0.492137 H, 4.521176, $-0.546334,-0.684814$ C, $-2.921533,0.771278,-0.086746$ $\mathrm{H},-2.768151,1.527982,0.675857$ H, $-2.66154,1.124981,-1.079489$ C, $-4.254541,0.089334,-0.017219$ $\mathrm{H},-4.467144,-0.294613,0.982918$ $\mathrm{H},-5.017009,0.844118,-0.232662$ H, $-4.353229,-0.697405,-0.765701$

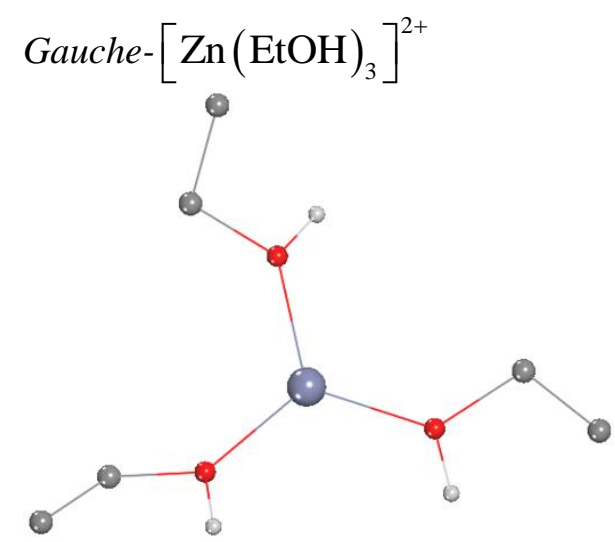

Zn, 0.177541, $-0.240635,-0.17535$ O, $-0.237834,1.616993,-0.408872$ O, 1.999316, $-0.833018,-0.211315$ H, 2.236328, $-1.753875,-0.415965$ $\mathrm{O},-1.264964,-1.451133,0.19674$

H, $-1.145643,-2.228948,0.768539$ $\mathrm{H}, 0.320342,2.212895,-0.93711$ C, 3.266527, $-0.013456,-0.085888$ $\mathrm{H}, 2.97926,0.823532,0.545199$ H, 3.503201, 0.319143, -1.093035 C, 4.344537, $-0.864432,0.528872$ $\mathrm{H}, 4.59638,-1.716335,-0.107145$ H, 5.248884, $-0.258879,0.621095$ $\mathrm{H}, 4.066879,-1.209604,1.52475$ C, $-2.632252,-1.51764,-0.449145$ $\mathrm{H},-2.875357,-0.479753,-0.661686$ $\mathrm{H},-2.498797,-2.077891,-1.370556$ C, $-3.5974,-2.163544,0.507655$ $\mathrm{H},-3.685765,-1.599331,1.436255$ $\mathrm{H},-4.581517,-2.19406,0.034828$ H, $-3.317698,-3.197215,0.724267$ C, $-1.472649,2.364834,0.041615$ $\mathrm{H},-2.161857,2.329052,-0.79819$ $\mathrm{H},-1.846788,1.777135,0.876303$ C, $-1.089304,3.765185,0.437277$ $\mathrm{H},-0.687863,4.326835,-0.409408$ $\mathrm{H},-1.989952,4.287805,0.767064$ $\mathrm{H},-0.375171,3.770134,1.260546$ 


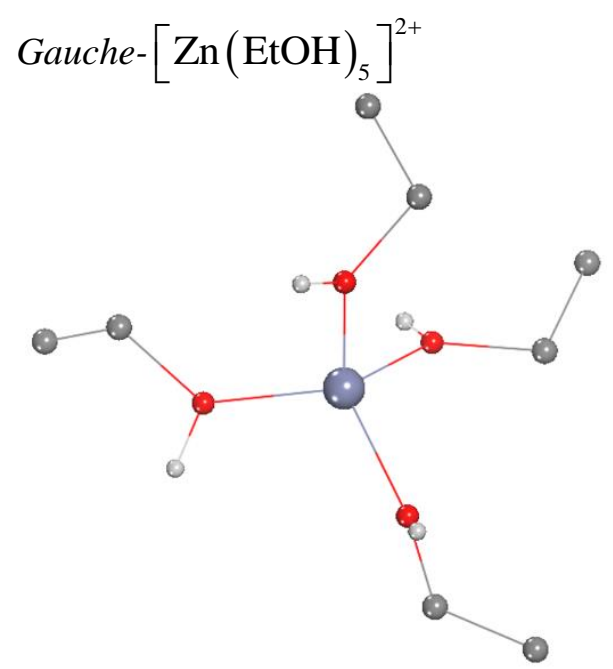

Zn, 0.092667, -0.299741, -0.224804

$\mathrm{O}, 1.411133,-1.681757,0.269026$

$\mathrm{O},-0.634159,0.518814,1.422594$

O, $-1.496775,-0.976611,-1.182891$

$\mathrm{H},-1.624774,-0.849768,-2.136416$

O, $1.029043,1.102423,-1.251217$

H, $-0.264383,0.328084,2.299385$

$\mathrm{H}, 1.549465,0.920562,-2.049716$

$\mathrm{H}, 1.18429,-2.624673,0.304048$

C, $2.894236,-1.52934,0.327384$

$\mathrm{H}, 3.047789,-0.491598,0.61297$

$\mathrm{H}, 3.267026,-1.700165,-0.6803$

C, $-2.558503,-1.874825,-0.651551$

$\mathrm{H},-2.301377,-2.885317,-0.961669$

$\mathrm{H},-2.46875,-1.785909,0.429064$

C, 0.901902, 2.576283, -1.066011

$\mathrm{H}, 0.064085,2.898005,-1.68091$

$\mathrm{H}, 0.663653,2.693966,-0.011668$

C, $-1.904375,1.27887,1.56214$

$\mathrm{H},-2.683943,0.55471,1.790644$

$\mathrm{H},-2.074892,1.705357,0.575888$

C, $-1.761508,2.331995,2.632357$

$\mathrm{H},-2.696934,2.889049,2.709357$

$\mathrm{H},-1.572438,1.883921,3.610554$

H, -0.963872, 3.037009, 2.396404

C, 2.195513, 3.252527, - 1.443847

$\mathrm{H}, 2.431973,3.101991,-2.499877$

$\mathrm{H}, 2.090599,4.328035,-1.289886$

$\mathrm{H}, 3.023985,2.900572,-0.828719$

C, $-3.908823,-1.434389,-1.157777$

$\mathrm{H},-3.971816,-1.511232,-2.245554$

$\mathrm{H},-4.674422,-2.093703,-0.744601$

H, $-4.135979,-0.412088,-0.853729$

C, 3.467708, $-2.497379,1.33087$

$\mathrm{H}, 4.550265,-2.363324,1.370936$
H, 3.066037, -2.323144, 2.329117

H, 3.28359, -3.533503, 1.037303

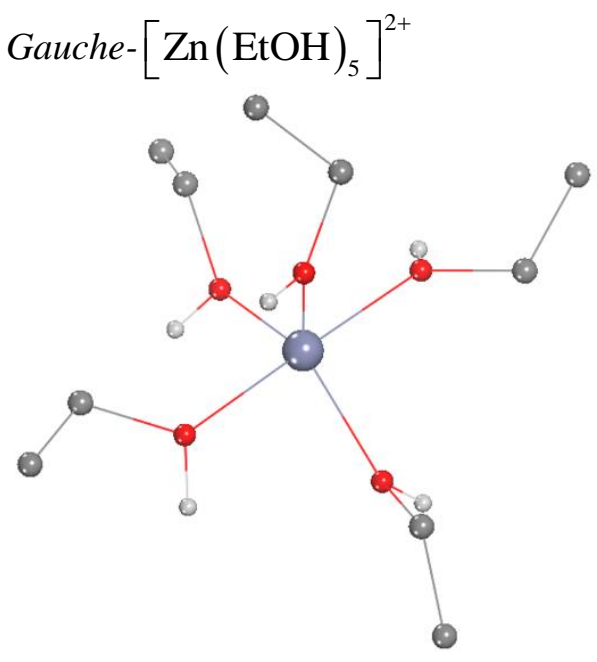

Zn, $-0.169595,-0.071798,-0.346718$

$\mathrm{O}, 0.111048,0.122564,1.640176$

$\mathrm{O},-1.5768,-1.466513,-0.851371$

O, 1.396929, - $1.391525,-0.552082$

$\mathrm{O}, 0.929815,1.190525,-1.515671$

O, $-1.767829,1.238942,-0.205944$

$\mathrm{H},-0.614069,0.502844,2.159185$

H, 1.997945, -1.301746, -1.307569

C, $-2.492077,-2.108823,0.114788$

C, $1.292342,-0.082072,2.498878$

C, $1.473376,-2.753046,-0.004301$

C, 2.116642, 1.928369, - 1.038801

C, $-1.717025,2.691048,0.015324$

$\mathrm{H}, 0.930143,-3.416402,-0.676946$

$\mathrm{H}, 2.620346,1.230055,-0.372808$

$\mathrm{H},-0.854943,2.839058,0.663771$

$\mathrm{H}, 2.069155,-0.411031,1.813667$

H, 0.946988, -2.694124, 0.946684

H, $-1.530861,3.170745,-0.945324$

C, 2.913356, $-3.177109,0.166718$

H, 2.947353, $-4.180798,0.593356$

H, 3.432582, $-3.215305,-0.793673$

$\mathrm{H}, 3.448772,-2.502832,0.836138$

C, $-2.998217,3.17797,0.651345$

$\mathrm{H},-2.930061,4.252717,0.827095$

H, -3.855297, 3.010191, -0.004712

$\mathrm{H},-3.17851,2.685678,1.607573$

C, $-3.675965,-2.710237,-0.603845$

$\mathrm{H},-3.365914,-3.503844,-1.287707$

$\mathrm{H},-4.35027,-3.158947,0.127461$ 
C, 2.98644, 2.336521, - 2.203587

H, 2.461556, 3.023896, - 2.871021

$\mathrm{H}, 3.867815,2.859577,-1.828783$

C, 1.656124, 1.197768, 3.213473

H, 2.54029, 1.028357, 3.830417

$\mathrm{H}, 1.881836,1.996914,2.505585$

$\mathrm{H}, 0.854738,1.524689,3.879796$

$\mathrm{H}, 1.051439,-0.889192,3.188649$

$\mathrm{H}, 3.320033,1.469067,-2.773682$

$\mathrm{H},-4.22973,-1.953824,-1.160661$

$\mathrm{H}, 0.537105,1.664173,-2.264829$

$\mathrm{H},-1.91965,-2.854323,0.665423$

$\mathrm{H},-2.783938,-1.30596,0.788957$

$\mathrm{H}, 1.763401,2.785487,-0.466271$

$\mathrm{H},-1.448854,-2.057246,-1.609379$

$\mathrm{H},-2.59683,1.001784,-0.649684$

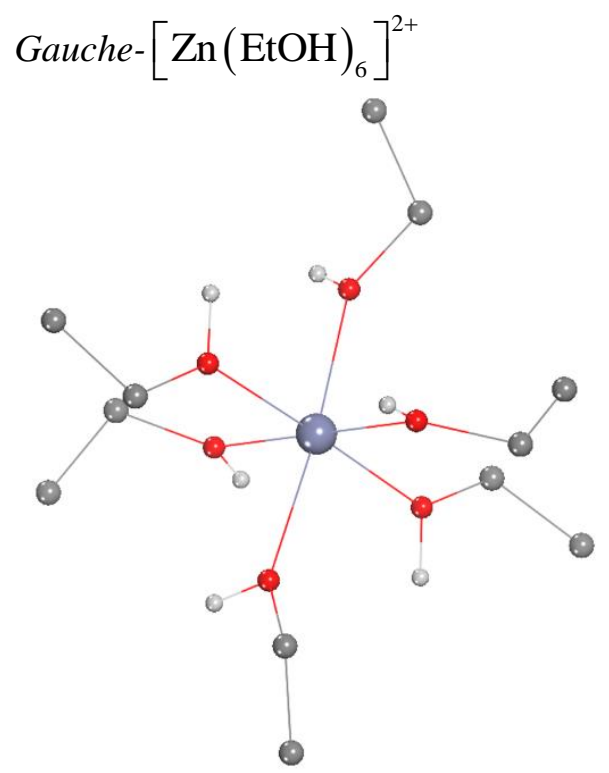

Zn, $-0.035659,-0.000705,-0.088965$

$\mathrm{O}, 1.316722,0.176621,1.532513$

$\mathrm{O},-1.503832,0.950857,1.142772$

O, $-0.680352,-1.980762,0.440162$

O, -1.419104, - $0.181808,-1.763353$

$\mathrm{O}, 1.38873,-0.992593,-1.339604$

$\mathrm{O}, 0.405775,1.957109,-0.837808$

C, 1.365045, 2.992134, -0.505701

$\mathrm{H}, 2.210544,2.890685,-1.172607$

$\mathrm{H}, 1.685206,2.766182,0.498814$

C, -2.485569, 0.510326, 2.113578

$\mathrm{H},-1.970115,0.32476,3.046603$

$\mathrm{H},-2.869811,-0.424591,1.735606$

C, 2.722108, -0.140989, 1.699475
H, 3.194204, 0.141903, 0.771768

H, 2.807302, -1.211918, 1.8227

C, $-0.461084,-2.867724,1.564877$

H, $-1.264665,-2.716577,2.272984$

H, 0.462192, - 2.533342, 2.011146

C, 2.335486, $-0.597736,-2.363159$

H, 2.604763, 0.420018, -2.127896

H, 1.821761, -0.610766, -3.315438

C, $-2.745593,0.315962,-2.069399$

H, -2.646562, 1.095974, -2.812931

$\mathrm{H},-3.097847,0.756413,-1.150691$

C, 3.316574, 0.602036, 2.877139

$\mathrm{H}, 2.838164,0.317112,3.809726$

$\mathrm{H}, 4.368435,0.355794,2.96624$

$\mathrm{H}, 3.232501,1.675985,2.753157$

C, $-3.659025,-0.79234,-2.548803$

H, $-3.775697,-1.564471,-1.795835$

$\mathrm{H},-4.640229,-0.385424,-2.766155$

$\mathrm{H},-3.287509,-1.248454,-3.461209$

C, $-0.375166,-4.312647,1.120493$

H, $-0.202596,-4.947096,1.982549$

H, -1.299562, -4.641637, 0.65557

H, 0.439905, $-4.465208,0.421695$

C, $0.745724,4.370568,-0.601679$

H, 1.485378, 5.118664, -0.339499

$\mathrm{H}, 0.40977,4.586884,-1.611215$

H, $-0.095047,4.477439,0.075$

C, 3.545982, -1.507234, -2.378913

H, 4.237455, -1.175732, -3.145113

H, 4.064947, $-1.49455,-1.427261$

$\mathrm{H}, 3.26975,-2.53088,-2.614218$

C, $-3.587688,1.533839,2.289019$

H, $-4.308846,1.168632,3.011108$

$\mathrm{H},-3.198932,2.474636,2.667547$

H, -4.110243, 1.719171, 1.357148

$\mathrm{H}, 0.926416,0.390253,2.372088$

$\mathrm{H},-0.019584,2.163942,-1.662537$

$\mathrm{H},-1.055732,-0.617209,-2.526476$

$\mathrm{H},-1.416116,-2.288947,-0.077543$

H, -1.463056, 1.900263, 1.12093

H, 1.368946, -1.939313, -1.25583 


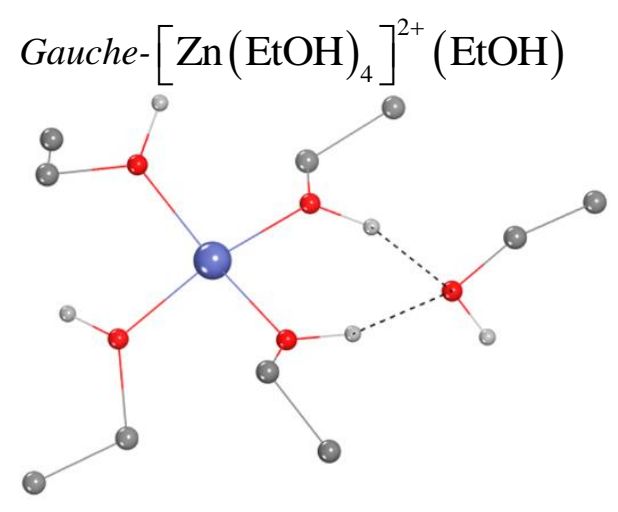

$\mathrm{H},-3.204055,0.914021,1.453975$

$\mathrm{H},-3.687697,2.37474,0.570161$

C, $-5.010121,0.682177,0.276962$

$\mathrm{H},-4.962337,-0.407205,0.252147$

$\mathrm{H},-5.69351,0.974203,1.075892$

$\mathrm{H},-5.434413,1.043521,-0.662598$

$\mathrm{H},-3.089904,1.116278,-1.37797$

Zn, 0.540883, $-0.430214,0.028298$

O, -1.184902, -1.34178, -0.178989

O, $-0.048999,1.432495,-0.172612$

O, 2.023284, $-0.710953,-1.257275$

H, 2.569561, -1.512444, -1.251689

O, 1.379587, $-0.951956,1.745991$

H, $-1.025018,1.512761,-0.307114$

H, 0.858268, -1.342464, 2.464434

H, $-1.931747,-0.725292,-0.371657$

C, $-1.487561,-2.724304,-0.598725$

$\mathrm{H},-0.799724,-3.34837,-0.033033$

$\mathrm{H},-1.2652,-2.800759,-1.661674$

C, 2.214814, 0.013779, - 2.540836

H, 1.635149, $-0.514799,-3.29479$

H, 1.781238, 0.9942, -2.360368

C, 2.775918, $-0.73051,2.197223$

H, 3.243036, -1.710929, 2.261548

H, 3.23244, $-0.160675,1.391109$

C, 0.529761, 2.656293, 0.408124

$\mathrm{H}, 1.605239,2.546989,0.281787$

$\mathrm{H}, 0.286261,2.665687,1.469727$

C, $-0.002417,3.871064,-0.314064$

$\mathrm{H}, 0.448136,4.769624,0.110312$

H, 0.239686, 3.835567, -1.376498

H, $-1.084513,3.960136,-0.193684$

C, 2.792221, 0.010867, 3.510995

$\mathrm{H}, 2.311548,-0.568228,4.302477$

$\mathrm{H}, 3.827821,0.174725,3.814386$

H, 2.302726, 0.981784, 3.426307

C, 3.683565, 0.081613, -2.877407

$\mathrm{H}, 4.105369,-0.914576,-3.029891$

$\mathrm{H}, 3.808438,0.629963,-3.812809$

$\mathrm{H}, 4.246294,0.599485,-2.100387$

C, $-2.929044,-3.050824,-0.291072$

$\mathrm{H},-3.129192,-4.087047,-0.568097$

$\mathrm{H},-3.140369,-2.935839,0.772626$

$\mathrm{H},-3.608842,-2.42163,-0.869714$

$\mathrm{O},-2.69195,0.907637,-0.518104$

C, $-3.648361,1.286418,0.532807$

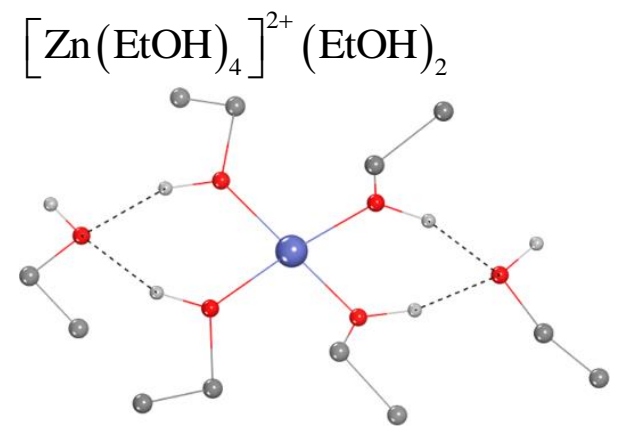

Zn, $-0.062294,0.33329,0.030963$

O, -1.518719, 0.917284, -1.160695

O, $-1.197477,-0.088036,1.586231$

O, $1.433743,1.580045,0.292287$

H, $2.31754,1.207075,0.061721$

O, $1.007212,-1.188488,-0.631928$

$\mathrm{H},-2.162948,0.025877,1.421323$

$\mathrm{H}, 1.968381,-1.005433,-0.751895$

H, $-2.418014,0.878415,-0.759599$

C, $-1.503491,1.714565,-2.395305$

H, $-0.525925,1.535764,-2.836992$

$\mathrm{H},-1.584752,2.762625,-2.11192$

C, $1.543193,2.776727,1.141089$

$\mathrm{H}, 0.567817,3.25418,1.086265$

$\mathrm{H}, 1.72683,2.441546,2.160684$

C, $0.552345,-2.333596,-1.431307$

H, - $0.386426,-2.641294,-0.975009$

H, 0.364807, -1.979094, -2.444081

C, $-0.943031,-1.047887,2.669644$

$\mathrm{H}, 0.060625,-0.821538,3.021801$

H, $-0.960247,-2.048559,2.238395$

C, $-1.975182,-0.877851,3.759477$

$\mathrm{H},-1.766414,-1.57819,4.56948$

$\mathrm{H},-1.951943,0.133164,4.166871$

$\mathrm{H},-2.98028,-1.095598,3.389406$

C, $1.584887,-3.435537,-1.394647$

H, 1.779213, -3.756869, - 0.370699

H, 1.219084, -4.294253, -1.959577

$\mathrm{H}, 2.520033,-3.11421,-1.858546$

C, 2.642045, 3.67546, 0.625306

H, 2.444542, 3.989696, - 0.399791 
$\mathrm{H}, 2.703266,4.56682,1.251544$

H, 3.613717, 3.177755, 0.669979

C, $-2.626112,1.275433,-3.305925$

H, $-2.603675,1.867903,-4.221913$

$\mathrm{H},-2.525426,0.223518,-3.575338$

$\mathrm{H},-3.600045,1.4358,-2.837215$

$\mathrm{O},-3.647886,0.486703,0.506572$

C, $-4.599836,-0.578776,0.178143$

$\mathrm{H},-5.168013,-0.815431,1.076644$

H, $-5.27581,-0.204982,-0.589696$

C, $-3.809045,-1.770585,-0.310045$

$\mathrm{H},-3.140371,-2.145453,0.469144$

H, $-4.493272,-2.577226,-0.575749$

H, $-3.226932,-1.517516,-1.199193$

$\mathrm{H},-4.131759,1.241789,0.874452$

O, 3.477125, -0.030328, -0.521732

H, 3.97129, 0.21792, -1.318474

C, 4.430304, $-0.532557,0.475039$

H, 5.014662, 0.313683, 0.835715

C, 5.309561, -1.617153, -0.105098

H, 5.996937, -1.978462, 0.661403

$\mathrm{H}, 4.715762,-2.462283,-0.455774$

H, 5.91385, -1.237616, -0.932225

H, 3.806925, -0.905128, 1.285878

\section{$\left[\mathrm{Zn}(\mathrm{EtOH})_{5}\right]^{2+}(\mathrm{EtOH})$}

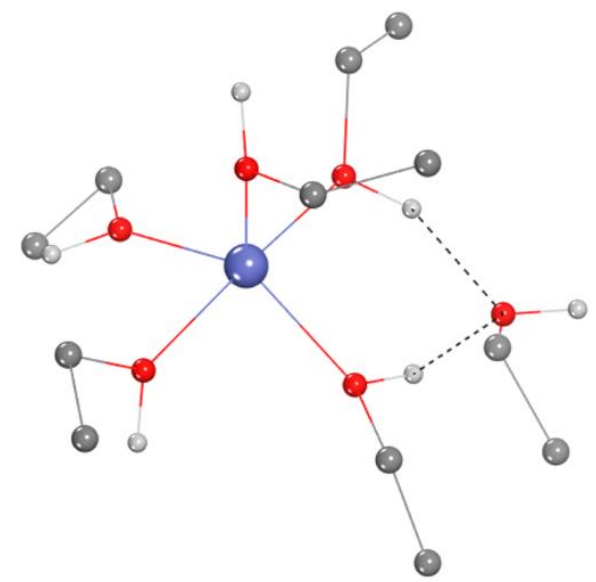

Zn, $-0.54497,0.386845,0.007746$

O, $-1.72979,0.602617,1.652853$

$\mathrm{O},-0.46388,2.141155,-1.009054$

$\mathrm{O},-2.25335,-0.052513,-1.126635$

O, $0.146134,-1.464682,-0.422125$

$\mathrm{O}, 1.261387,0.809925,0.885572$

$\mathrm{H},-1.5868,1.33324,2.272791$

$\mathrm{H},-2.1307,-0.495106,-1.979942$

C, $0.590315,3.139433,-1.245946$
C, $-2.21754,-0.579937,2.376309$

C, $-3.67724,0.270719,-0.947719$

C, $-0.54434,-2.756932,-0.407858$

C, $1.523291,1.78469,1.937433$

$\mathrm{H},-3.93594,1.041903,-1.672696$

$\mathrm{H},-1.5761,-2.535908,-0.670019$

$\mathrm{H}, 0.895173,2.642991,1.700238$

$\mathrm{H},-2.50189,-1.276722,1.589595$

$\mathrm{H},-3.7382,0.685837,0.05412$

$\mathrm{H}, 1.203153,1.355273,2.887988$

C, $-4.52965,-0.966368,-1.11359$

$\mathrm{H},-5.57949,-0.706386,-0.968155$

H, $-4.43513,-1.384205,-2.118614$

$\mathrm{H},-4.26484,-1.732196,-0.382821$

C, 2.988526, 2.161001, 1.962304

H, 3.164857, 2.906134, 2.739306

H, 3.611928, 1.293634, 2.191025

H, 3.302279, 2.581908, 1.00608

C, $0.661289,3.493081,-2.713273$

$\mathrm{H},-0.26918,3.95027,-3.058437$

$\mathrm{H}, 1.456248,4.223768,-2.87088$

C, 0.098618, -3.710523, - 1.389568

$\mathrm{H}, 1.13237,-3.925098,-1.111161$

H, $-0.44504,-4.656675,-1.388406$

C, $-1.14614,-1.141308,3.282758$

$\mathrm{H},-1.51708,-2.040873,3.777253$

$\mathrm{H},-0.25176,-1.404962,2.712664$

$\mathrm{H},-0.8725,-0.427668,4.06246$

$\mathrm{H},-3.112,-0.280999,2.919742$

H, $0.080649,-3.303807,-2.401224$

$\mathrm{H}, 0.878122,2.61449,-3.321904$

H, 1.106783, $-1.602999,-0.250523$

$\mathrm{H}, 0.361388,4.005025,-0.625853$

$\mathrm{H}, 1.500494,2.663987,-0.892115$

$\mathrm{H},-0.51346,-3.141045,0.612476$

$\mathrm{H},-1.25045,2.365969,-1.527592$

$\mathrm{H}, 1.939609,0.097282,0.889383$

O, 2.78934, -1.380976, 0.322514

H, 3.133841, -2.027223, 0.95748

C, 3.832523, $-1.099283,-0.668801$

H, 3.363729, $-0.410178,-1.369154$

H, 4.647715, $-0.585327,-0.159909$

C, $4.298547,-2.365859,-1.350645$

H, 5.066757, - $2.123299,-2.086664$

H, 4.740974, $-3.061974,-0.634498$

H, 3.475515, -2.860894, - 1.867819 


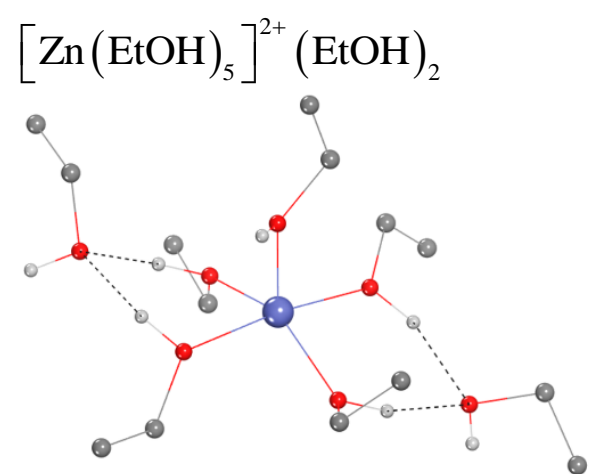

Zn, $-0.02499,-0.134768,-0.15139$

$\mathrm{O},-1.19136,-1.78777,-0.643658$

O, -1.4961, 1.092462, -0.840589

$\mathrm{O}, 1.201088,1.496617,0.040422$

$\mathrm{O}, 1.628398,-1.217036,-0.634468$

$\mathrm{O},-0.56536,-0.494643,1.786961$

H, $-2.13433,-1.589529,-0.456103$

H, 2.15123, 1.385532, -0.185193

C, $-1.43177,2.001657,-1.982361$

C, $-1.12616,-2.448966,-1.947808$

C, 0.936987, 2.838911, 0.546299

C, 1.90886, -2.570512, -0.163432

C, $-0.17039,0.287737,2.963466$

$\mathrm{H}, 1.232194,2.86523,1.595407$

$\mathrm{H}, 2.465313,-3.080591,-0.948941$

H, 0.918269, 0.282068, 3.018628

$\mathrm{H},-0.06815,-2.505507,-2.191749$

$\mathrm{H},-0.14144,2.966253,0.47706$

$\mathrm{H},-0.51976,1.296066,2.754633$

C, 1.6861, 3.876079, -0.262182

$\mathrm{H}, 1.465726,4.871914,0.125269$

$\mathrm{H}, 2.765885,3.727523,-0.187626$

$\mathrm{H}, 1.398287,3.847267,-1.314136$

C, $-0.80715,-0.281466,4.209927$

$\mathrm{H},-0.5265,0.324756,5.072383$

$\mathrm{H},-1.89515,-0.277903,4.132532$

$\mathrm{H},-0.46276,-1.300705,4.402995$

C, $-2.47463,3.089287,-1.852546$

$\mathrm{H},-2.33526,3.658098,-0.932162$

H, $-2.39739,3.775604,-2.69736$

C, 2.668026, $-2.548531,1.146232$

H, 2.113039, -2.002467, 1.912906

H, 2.826382, -3.569046, 1.498937

C, $-1.76656,-3.817211,-1.878145$

$\mathrm{H},-1.70137,-4.304919,-2.852097$

H, $-1.26178,-4.445966,-1.143771$

$\mathrm{H},-2.82282,-3.74686,-1.610182$

H, -1.61743, -1.804256, -2.679243

H, 3.64926, -2.085149, 1.028193

H, $-3.48351,2.671085,-1.859026$
$\mathrm{H}, 2.467602,-0.716961,-0.752044$

H, - $0.4212,2.402596,-1.968121$

$\mathrm{H},-1.5648,1.416588,-2.893129$

H, 0.935949, -3.045066, -0.058097

$\mathrm{H},-2.38729,0.680504,-0.781891$

$\mathrm{H},-0.66079,-1.429439,2.025657$

$\mathrm{O}, 3.671698,0.624943,-0.79643$

C, 4.848172, 0.631697, 0.074071

H, 5.191892, 1.661563, 0.169139

$\mathrm{H}, 4.476134,0.29669,1.040662$

C, 5.932122, $-0.276118,-0.463529$

H, 6.291994, 0.070002, - 1.43495

H, 6.783293, $-0.277301,0.219194$

H, 5.574298, $-1.301965,-0.567401$

$\mathrm{H}, 3.950165,0.872348,-1.691306$

O, -3.665566, $-0.545048,-0.403357$

$\mathrm{H},-4.342156,-0.722567,-1.074196$

C, $-4.33565,-0.311696,0.875485$

$\mathrm{H},-3.526249,-0.0862,1.568192$

H, $-4.814629,-1.241456,1.181445$

C, $-5.324629,0.828958,0.775562$

$\mathrm{H},-6.11705,0.60379,0.058268$

H, $-5.799029,0.992193,1.744635$

H, $-4.828609,1.754059,0.477008$
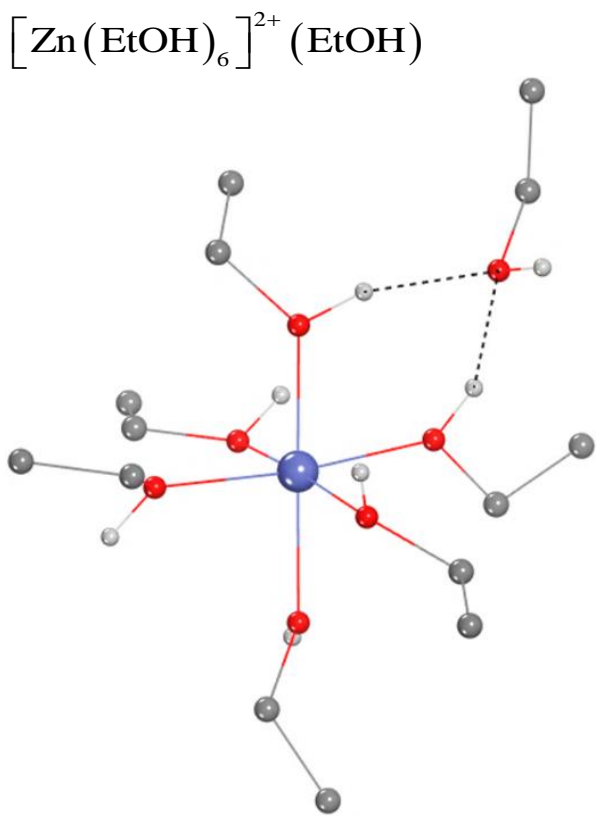

Zn, $0.460894,0.123648,0.074463$

O, -1.072957, 0.846133, - 1.150463

O, - $0.929301,-1.189096,0.921737$

$\mathrm{O}, 0.046908,1.276102,1.78476$

$\mathrm{O}, 2.083115,-0.66494,1.151415$ 
O, 1.689399, 1.62684, - 0.730845

O, $0.973675,-1.207496,-1.470648$

C, 1.061234, -2.657479, - 1.331976

H, 0.04946, $-3.066272,-1.345871$

$\mathrm{H}, 1.487114,-2.809858,-0.343234$

C, $-0.734204,-2.190027,1.960109$

$\mathrm{H},-0.260188,-1.664922,2.787976$

H, $-0.048136,-2.949749,1.585097$

C, $-1.455262,2.236259,-1.350392$

H, -1.719566, 2.360994, -2.400589

$\mathrm{H},-0.55472,2.810663,-1.144764$

C, $0.422433,2.648512,2.113673$

H, 0.088072, 3.297641, 1.304174

$\mathrm{H}, 1.510747,2.645101,2.14311$

C, 2.011695, 1.7663, -2.150988

$\mathrm{H}, 1.069263,1.585015,-2.664293$

$\mathrm{H}, 2.723703,0.98418,-2.415947$

C, 3.449075, $-0.891789,0.68626$

$\mathrm{H}, 4.029777,0.013274,0.872346$

H, 3.349545, -1.034234, -0.387565

C, $-2.596798,2.639945,-0.441084$

$\mathrm{H},-2.331514,2.505039,0.609726$

$\mathrm{H},-2.840457,3.692093,-0.597994$

$\mathrm{H},-3.497016,2.060012,-0.654143$

C, $4.063095,-2.092662,1.370965$

$\mathrm{H}, 3.478061,-2.99445,1.185498$

$\mathrm{H}, 5.073268,-2.25444,0.991348$

H, 4.139222, -1.938389, 2.449513

C, $-0.16291,3.058138,3.446315$

$\mathrm{H}, 0.153894,4.073062,3.69028$

$\mathrm{H},-1.255763,3.056936,3.415442$

H, 0.173601, 2.395017, 4.244096

C, $1.927552,-3.251466,-2.420165$

H, 1.999946, $-4.331741,-2.284452$

H, 1.501659, $-3.073408,-3.41034$

$\mathrm{H}, 2.934715,-2.833649,-2.390538$

C, 2.549366, 3.148971, -2.4433

H, 2.757527, 3.243398, -3.510151

H, 1.827548, 3.917655, -2.164582

H, 3.486313, 3.331631, -1.911513

C, $-2.059514,-2.791032,2.375705$

H, -1.902516, -3.514995, 3.176344

H, $-2.741057,-2.021308,2.741693$

$\mathrm{H},-2.529472,-3.319643,1.54262$

H, $-1.857179,0.260337,-1.219906$

H, 0.665013, - $0.982114,-2.360168$

$\mathrm{H}, 2.074635,-0.543192,2.111979$

$\mathrm{H},-0.848381,1.0911,2.106048$

H, -1.672147, $-1.435644,0.330426$

H, 2.444755, 1.931098, - 0.207756

O, - $2.980477,-1.184162,-0.929512$
C, $-4.283237,-0.950383,-0.306888$

$\mathrm{H},-4.580944,-1.86183,0.211799$

H, $-4.098847,-0.172494,0.432506$

C, $-5.31363,-0.527571,-1.330803$

$\mathrm{H},-6.264801,-0.326428,-0.835447$

$\mathrm{H},-5.002838,0.376311,-1.857359$

H, $-5.488497,-1.318075,-2.064281$

H, $-3.106863,-1.794482,-1.671151$

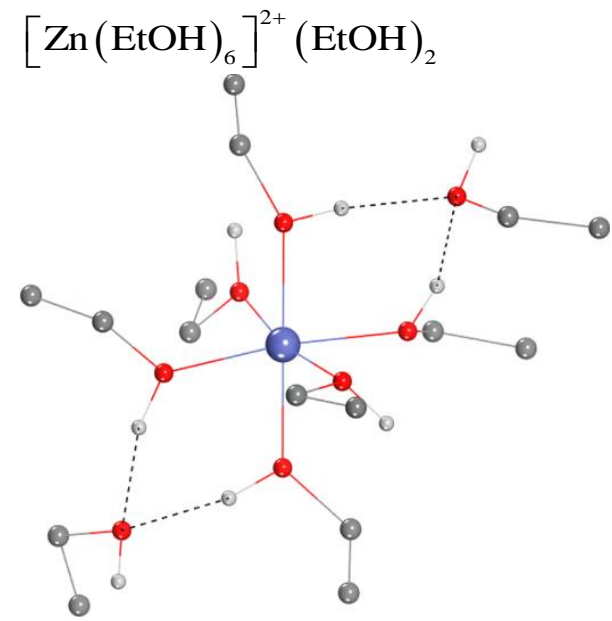

Zn, $0.000000,0.000000,0.000000$

$\mathrm{O}, 0.000000,0.000000,2.08250726$

O, 2.09294574, 0.000000, 0.12362431

O, $-0.18310969,2.11456215,-0.10145012$

O, 0.17583444, 0.20244669, - 2.07987126

O, -2.09555719, 0.0491952, -0.10916497

O, $-0.03128972,-2.10197167,-0.18117041$

C, $0.93578666,-3.07987427,0.29851231$

$\mathrm{H}, 0.71595403,-3.2889179,1.34668723$

H, $1.89705092,-2.57698345,0.22191776$

C, $2.96830299,0.89607224,-0.61476008$

$\mathrm{H}, 3.01222473,1.85092551,-0.08526764$

H, 2.48117102, 1.0461088, - 1.5756633

C, $-1.07761139,-0.08581613,3.05061338$

H, $-1.98392384,0.1439046,2.49525322$

$\mathrm{H},-0.92463809,0.69598321,3.79548259$

C, $-0.53928372,2.93603976,1.04842984$

$\mathrm{H}, 0.26310443,2.86468301,1.78352494$

H, -1.43219868, 2.46497988, 1.45257939

C, $-3.0804219,-0.98648756,0.13544495$

$\mathrm{H},-2.70572879,-1.55183968,0.98790748$

H, $-3.12392215,-1.64524473,-0.73662453$

C, $0.77685628,-0.80286072,-2.93967604$

$\mathrm{H}, 0.07893408,-1.63458116,-3.04625378$

$\mathrm{H}, 1.65027862,-1.15845351,-2.39501435$

C, $-1.13115254,-1.4593331,3.68666697$ 
H, $-0.21154758,-1.67265875,4.23638175$

$\mathrm{H},-1.95959601,-1.5133413,4.39503784$

H, $-1.27265974,-2.23429788,2.93044432$

C, $1.15100427,-0.2069244,-4.28003874$

$\mathrm{H}, 1.8481464,0.62349848,-4.15806677$

H, 1.62568418, - $0.9651851,-4.90486374$

$\mathrm{H}, 0.26722655,0.15386218,-4.81109565$

C, $-0.79774656,4.36742746,0.63286075$

$\mathrm{H},-1.0658518,4.96127337,1.50803496$

$\mathrm{H}, 0.09313016,4.82177348,0.19144477$

H, -1.61899829, 4.42755765, -0.08202544

C, $0.8918621,-4.3346007,-0.54617257$

$\mathrm{H}, 1.63644439,-5.04760876,-0.18882488$

H, - $0.08349637,-4.82353893,-0.48149979$

$\mathrm{H}, 1.10766784,-4.10851458,-1.59115596$

C, $-4.43671648,-0.38155065,0.4310562$

$\mathrm{H},-5.16280688,-1.17098416,0.63091427$

$\mathrm{H},-4.38665291,0.27204388,1.30281442$

H, $-4.79935691,0.19925163,-0.41962712$

C, 4.34616629, 0.28972613, -0.77558884

$\mathrm{H}, 4.98213177,0.95998392,-1.35611398$

$\mathrm{H}, 4.82440634,0.13565309,0.19408008$

H, 4.28891488, - $0.66764573,-1.29571342$

$\mathrm{H}, 0.86718359,-0.0816986,2.53085574$

H, $-0.87691233,-2.54167138,-0.34625298$

$\mathrm{H},-0.64643421,0.54852982,-2.48779599$

$\mathrm{H}, 0.48577948,2.56822354,-0.63428055$

$\mathrm{H}, 2.46025367,-0.13872593,1.02258918$

$\mathrm{H},-2.35989842,0.55856644,-0.90523639$

$\mathrm{O},-2.38269853,1.14084724,-2.66472757$

H, $-2.93216364,0.65432654,-3.29698435$

O, 2.65271395, - $0.29089526,2.83862921$

H, 3.14806594, 0.43255305, 3.24983896

C, $-2.5373976,2.56853041,-2.92959998$

H, $-3.56982415,2.84512972,-2.71543782$

$\mathrm{H},-1.88703333,3.05023232,-2.2028188$

C, $-2.14529601,2.90847652,-4.35126354$

H, $-2.78848409,2.39866177,-5.07221881$

H, $-2.25398992,3.98136655,-4.51847748$

H, -1.1073482, 2.63437983, -4.54819092

C, $3.11365489,-1.55481377,3.40791374$

H, 2.41140118, $-2.29741818,3.03360633$

H, 3.01233442, -1.49726636, 4.49144704

C, $4.53378715,-1.8628151,2.98598168$

H, 5.22632926, -1.09517756, 3.33776133

H, 4.84968815, -2.81371196, 3.41836838

H, 4.61175075, -1.93796052, 1.89973736

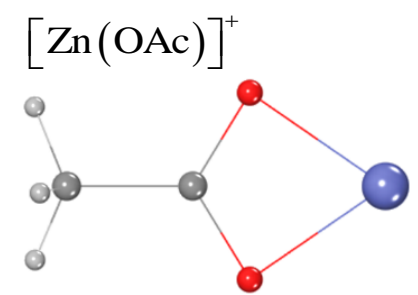

Zn, -1.214849, - $0.000566,0.003491$

O, $0.383769,1.097808,-0.008472$

C, $1.056329,0.001873,-0.013952$

$\mathrm{O}, 0.385158,-1.095572,-0.008578$

C, 2.543451, 0.000508, 0.002649

$\mathrm{H}, 2.858331,-0.080373,1.047162$

$\mathrm{H}, 2.91601,-0.866801,-0.537477$

H, 2.921034, 0.931992, -0.41019

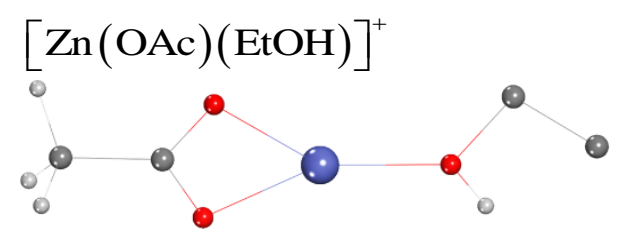

Zn, $-0.055358,-0.267711,-0.062138$

O, - $1.456586,1.107574,-0.055455$

C, $-2.31121,0.15771,0.046121$

$\mathrm{O},-1.846692,-1.037456,0.085173$

C, $-3.770088,0.427883,0.143885$

$\mathrm{H},-4.332781,-0.406911,-0.266661$

H, -4.020526, 0.525996, 1.203638

O, 1.829364, $-0.504047,-0.140929$

H, 2.244486, -1.382044, - 0.134625

C, $2.854879,0.56246,-0.375601$

H, 3.06977, 0.547081, -1.440977

H, 2.346207, 1.485167, -0.108563

C, 4.062639, 0.29349, 0.483321

$\mathrm{H}, 4.793799,1.085944,0.312718$

$\mathrm{H}, 3.804341,0.287957,1.542098$

$\mathrm{H}, 4.54129,-0.651588,0.216793$

$\mathrm{H},-4.011854,1.36189,-0.356956$ 

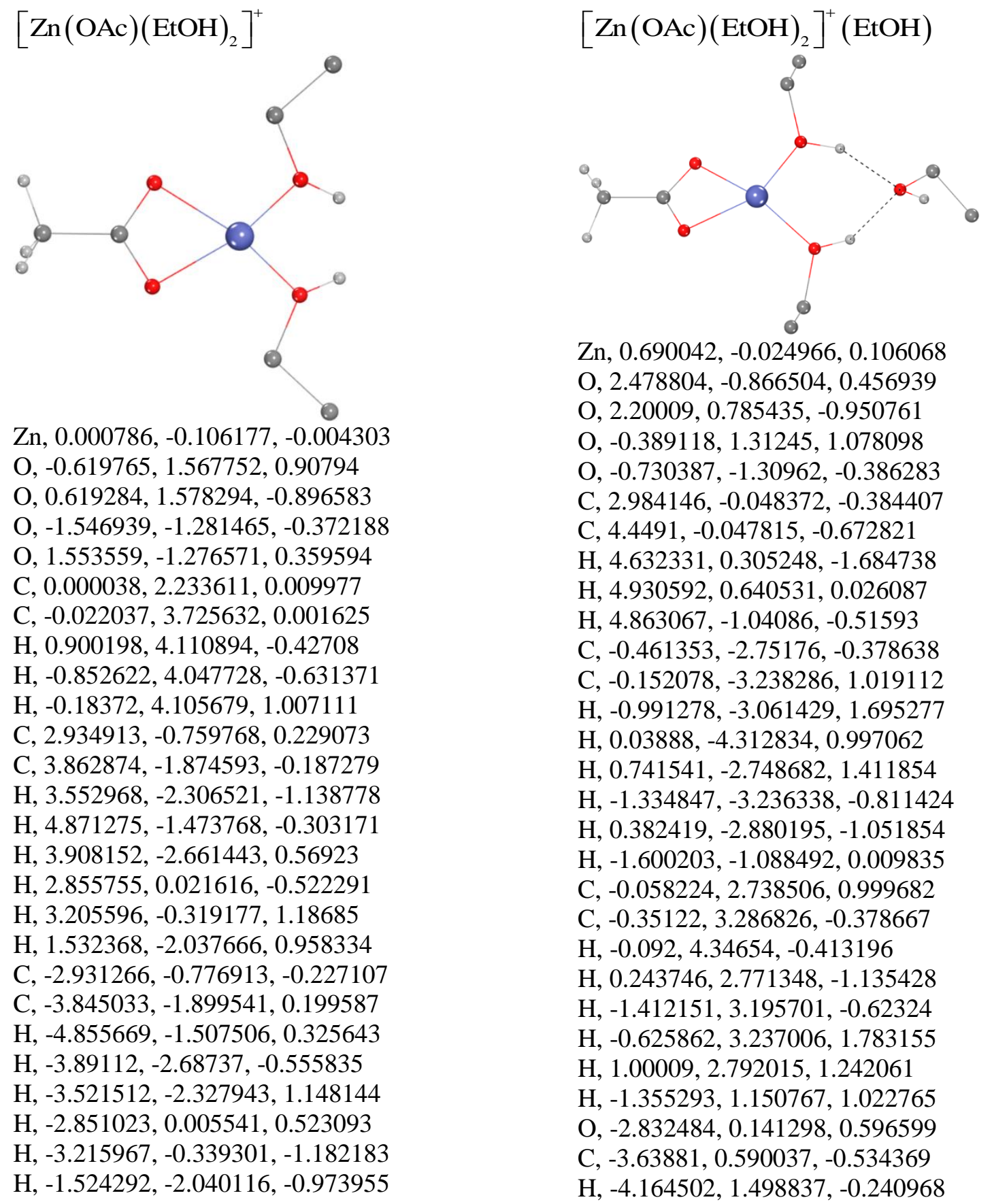

Zn, 0.690042, -0.024966, 0.106068

O, 2.478804, - $0.866504,0.456939$

$\mathrm{O}, 2.20009,0.785435,-0.950761$

O, $-0.389118,1.31245,1.078098$

O, $-0.730387,-1.30962,-0.386283$

C, $2.984146,-0.048372,-0.384407$

C, $4.4491,-0.047815,-0.672821$

$\mathrm{H}, 4.632331,0.305248,-1.684738$

$\mathrm{H}, 4.930592,0.640531,0.026087$

H, 4.863067, -1.04086, -0.51593

C, $-0.461353,-2.75176,-0.378638$

C, $-0.152078,-3.238286,1.019112$

H, $-0.991278,-3.061429,1.695277$

$\mathrm{H}, 0.03888,-4.312834,0.997062$

$\mathrm{H}, 0.741541,-2.748682,1.411854$

$\mathrm{H},-1.334847,-3.236338,-0.811424$

H, 0.382419, -2.880195, -1.051854

H, $-1.600203,-1.088492,0.009835$

C, $-0.058224,2.738506,0.999682$

C, $-0.35122,3.286826,-0.378667$

H, $-0.092,4.34654,-0.413196$

$\mathrm{H}, 0.243746,2.771348,-1.135428$

$\mathrm{H},-1.412151,3.195701,-0.62324$

$\mathrm{H},-0.625862,3.237006,1.783155$

$\mathrm{H}, 1.00009,2.792015,1.242061$

$\mathrm{H},-1.355293,1.150767,1.022765$

$\mathrm{O},-2.832484,0.141298,0.596599$

C, $-3.63881,0.590037,-0.534369$

$\mathrm{H},-4.164502,1.498837,-0.240968$

$\mathrm{H},-2.913404,0.837178,-1.30778$

C, $-4.592087,-0.492958,-0.991334$

$\mathrm{H},-5.302313,-0.75379,-0.203546$

$\mathrm{H},-5.165169,-0.141283,-1.85069$

$\mathrm{H},-4.048754,-1.391447,-1.289619$

H, $-3.420249,-0.092392,1.330201$ 


$$
\left[\mathrm{Zn}(\mathrm{OAc})(\mathrm{EtOH})_{2}\right]^{+}(\mathrm{EtOH})_{2}
$$

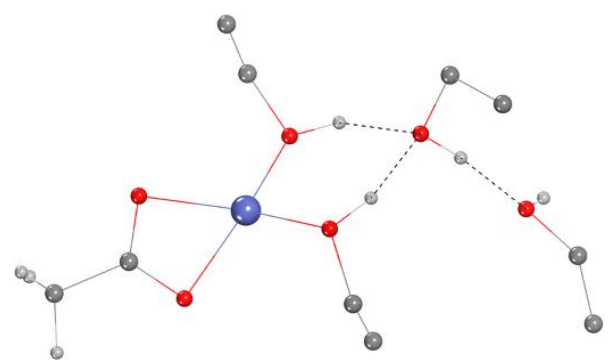

Zn, -1.46913, -0.119147, 0.068341

$\mathrm{O},-2.59695,-1.736211,-0.348986$

$\mathrm{O},-3.43687,0.145494,0.388625$

O, $-0.66248,1.242935,-1.115573$

$\mathrm{O}, 0.072969,-0.457416,1.254462$

C, $-3.61962,-1.058509,0.003351$

C, $-4.99317,-1.642065,-0.058699$

$\mathrm{H},-5.59991,-1.251907,0.755634$

$\mathrm{H},-5.45073,-1.332516,-1.001359$

H, $-4.94435,-2.727443,-0.033048$

C, $0.502752,-1.833348,1.493759$

C, $0.871962,-2.515941,0.193451$

$\mathrm{H}, 1.662614,-1.969161,-0.326677$

H, 1.230623, -3.527305, 0.39541

H, $0.001226,-2.598132,-0.461628$

H, 1.33646, $-1.786018,2.193439$

H, $-0.33902,-2.316435,1.983515$

$\mathrm{H}, 0.834762,0.107755,0.964827$

C, $-1.4393,2.38766,-1.588881$

C, $-1.8235,3.29492,-0.440994$

$\mathrm{H},-2.40302,4.139554,-0.818154$

$\mathrm{H},-2.44267,2.761487,0.283523$

H, $-0.93854,3.69327,0.060392$

$\mathrm{H},-0.83441,2.892907,-2.339835$

H, $-2.31156,1.954922,-2.072543$

H, $0.248537,1.491886,-0.833209$

$\mathrm{O}, 1.748272,1.287123,0.119401$

C, 2.284927, 2.361236, 0.932434

H, 2.759099, 3.088785, 0.272816

H, 1.422295, 2.82943, 1.404324

C, 3.256981, 1.830881, 1.967046

H, 4.116693, 1.359393, 1.486969

H, 3.625471, 2.649118, 2.588013

$\mathrm{H}, 2.77013,1.102834,2.619631$

H, 2.482832, $0.846084,-0.374298$

O, 3.687022, - $0.093176,-1.153898$

H, 4.00722, 0.109306, - 2.042992

C, 4.637056, $-0.948209,-0.482324$

H, 5.549308, $-0.382792,-0.282468$

H, 4.169471, -1.192997, 0.471208
C, $4.924482,-2.198364,-1.289356$

H, 5.61531, $-2.844923,-0.745521$

H, 4.005576, $-2.75349,-1.482974$

H, 5.389246, $-1.948749,-2.246201$

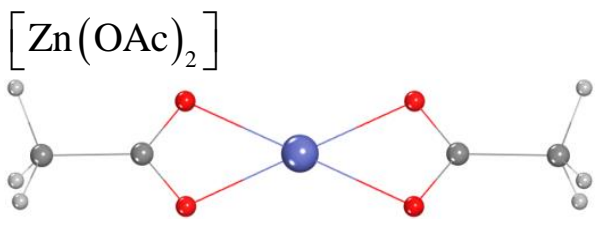

Zn, 0, $0.008653,-0.000003$

O, 1.698132, $-0.769351,0.783929$

$\mathrm{O},-1.70841,0.779545,0.763675$

C, $2.352508,0.003463,0.013285$

C, $-2.35251,0.003472,-0.01328$

$\mathrm{O}, 1.708417,0.779694,-0.763516$

O, -1.69814, $-0.769186,-0.784085$

C, $3.849872,-0.019922,-0.004379$

C, $-3.84987,-0.019925,0.004384$

H, 4.167918, $-0.696737,-0.800558$

H, 4.23471, 0.973098, -0.226174

H, 4.231681, $-0.390209,0.943755$

H, -4.16791, -0.697058, 0.800297

$\mathrm{H},-4.23472,0.973005,0.22656$

H, $-4.23168,-0.389856,-0.94389$

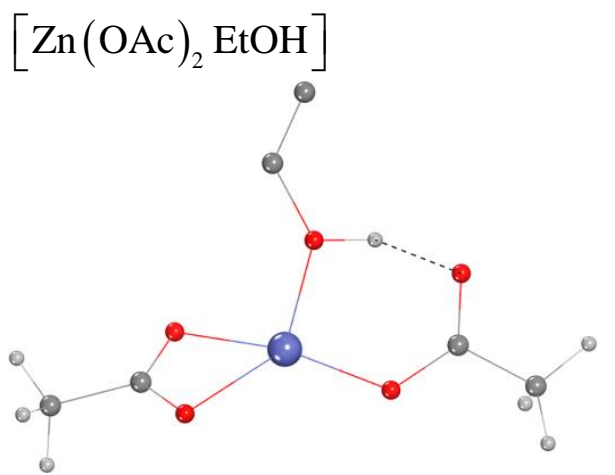

Zn, $0.265138,-0.425677,-0.160789$

$\mathrm{O},-0.50474,1.401998,-0.435382$

$\mathrm{O},-2.73603,0.198954,0.008338$

$\mathrm{O}, 1.9096,-0.232665,1.070227$

O, 2.046495, -0.85204, -1.02881

O, $-1.29023,-1.519384,0.004443$

C, $2.6179,-0.590861,0.078701$

C, $-2.47899,-1.015871,0.078821$

C, $-0.10176,2.524289,0.378739$

$\mathrm{H}, 0.978567,2.598346,0.270521$ 
H, $-0.32994,2.305628,1.423971$

C, $-0.80008,3.783502,-0.092902$

H, $-0.47618,4.637934,0.505096$

H, $-0.56442,3.977333,-1.139868$

$\mathrm{H},-1.88258,3.684838,0.009043$

$\mathrm{H},-1.47185,1.152698,-0.281945$

C, 4.105033, -0.736248, 0.209546

$\mathrm{H}, 4.471692,-0.14284,1.043465$

$\mathrm{H}, 4.326695,-1.788566,0.400453$

$\mathrm{H}, 4.589995,-0.451544,-0.722059$

C, $-3.5872,-2.02186,0.258301$

$\mathrm{H},-3.586,-2.705824,-0.591646$

$\mathrm{H},-3.39376,-2.612947,1.154223$

$\mathrm{H},-4.54655,-1.517352,0.334674$

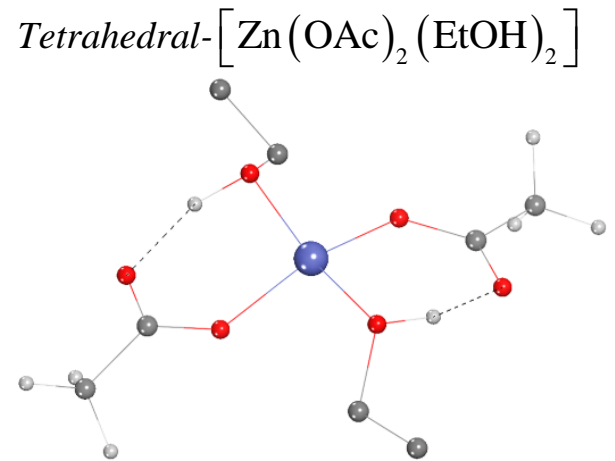

Zn, 0.100597, 0.105693, -0.504698

O, -1.111194, 1.656312, -0.076725

$\mathrm{O},-2.97703,-0.081581,-0.474208$

$\mathrm{O}, 0.708869,-0.52034,1.334125$

$\mathrm{O}, 1.783598,0.515282,-1.340252$

O, -1.161125, -1.204674, - 1.165593

C, 2.920839, 0.257796, -0.791002

C, $-2.432508,-1.070614,-1.001177$

C, $-1.044025,2.308673,1.2075$

H, $-0.029566,2.694782,1.295219$

$\mathrm{H},-1.199166,1.566471,1.993097$

C, $-2.070172,3.421861,1.278575$

H, $-2.003454,3.934067,2.240842$

H, $-1.8984,4.146333,0.481729$

H, $-3.080106,3.020428,1.173128$

$\mathrm{H},-1.977036,1.145967,-0.17721$

C, $-3.272552,-2.218616,-1.504913$

$\mathrm{H},-3.125183,-2.317236,-2.581543$

$\mathrm{H},-2.932928,-3.146351,-1.042684$

$\mathrm{H},-4.322554,-2.044997,-1.285003$

$\mathrm{O}, 3.064413,-0.226561,0.348148$

C, 4.129491, 0.58838, -1.631004

H, 5.04214, 0.339236, -1.096254
$\mathrm{H}, 4.074612,0.033887,-2.568763$

$\mathrm{H}, 4.112137,1.650777,-1.878047$

H, 1.701701, $-0.474511,1.138053$

C, 0.331428, -1.83295, 1.794276

H, $-0.757244,-1.835351,1.832808$

H, 0.650921, - 2.575862, 1.059929

C, $0.93725,-2.103711,3.157197$

$\mathrm{H}, 0.630453,-3.088135,3.517103$

$\mathrm{H}, 2.027518,-2.082634,3.103307$

H, 0.609489, -1.350076, 3.874526

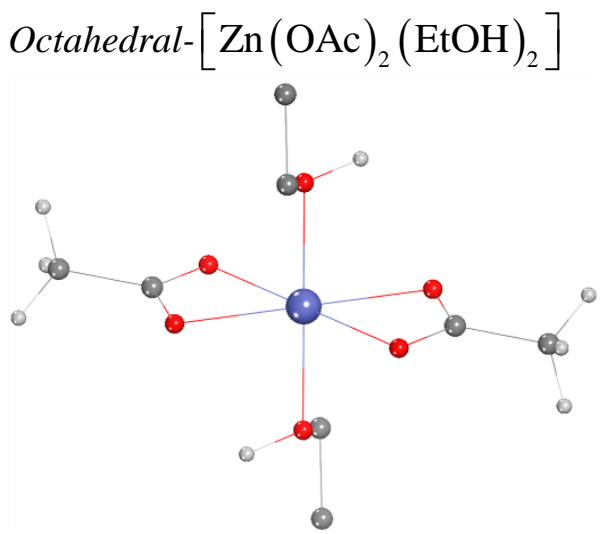

Zn, 0, 0, 0

O, 0.384596, - $1.775444,-1.053036$

$\mathrm{O}, 0.309648,1.832896,-1.034305$

C, $0.099767,-2.43305,0.000228$

C, $-0.099768,2.433049,-0.000229$

O, $-0.309648,-1.832897,1.034305$

O, $-0.384596,1.775444,1.053036$

C, $0.282526,-3.927707,0.013778$

C, $-0.282525,3.927707,-0.013779$

$\mathrm{H}, 1.316135,-4.138697,0.297815$

H, $-0.376347,-4.383898,0.749544$

H, $0.111392,-4.339802,-0.978925$

$\mathrm{H},-0.111391,4.339802,0.978924$

H, $0.376347,4.383898,-0.749546$

H, $-1.316135,4.138697,-0.297816$

$\mathrm{O}, 2.049775,0.068317,0.640322$

O, $-2.049775,-0.068317,-0.640322$

H, 2.114888, 0.839137, 1.222697

H, -2.114889, $-0.839137,-1.222696$

C, 3.016589, 0.172438, -0.430758

H, 2.80976, 1.075787, - 1.006029

H, 2.816518, $-0.692609,-1.0591$

C, $-3.016588,-0.172438,0.430759$

$\mathrm{H},-2.80976,-1.075787,1.00603$

H, $-2.816517,0.69261,1.059101$

C, 4.429183, 0.15248, 0.119334 
$\mathrm{H}, 4.600043,-0.754907,0.699869$

$\mathrm{H}, 5.149671,0.185563,-0.70067$

$\mathrm{H}, 4.614065,1.017377,0.761913$

C, $-4.429183,-0.152479,-0.119332$

H, -4.614066, -1.017376, -0.761911

$\mathrm{H},-5.149671,-0.185562,0.700673$

H, -4.600043, 0.754908, - 0.699867 
Optimized geometries of Zinc complexes in solution phase at the M05-2X/6-31 + G(d)/SMD level of theory.

Gauche- $(\mathrm{EtOH})_{2}$
C, $-2.19606,0.947441,-0.513066$
C, $-2.265769,-0.397512,0.189226$
$\mathrm{O},-1.077412,-1.169545,-0.010879$
$\mathrm{H},-3.137944,1.488742,-0.387562$
$\mathrm{H},-1.392662,1.560711,-0.097391$
$\mathrm{H},-2.014417,0.811118,-1.582009$
$\mathrm{H},-3.084596,-0.995437,-0.211535$
$\mathrm{H},-2.441303,-0.260056,1.260739$
$\mathrm{H},-0.33342,-0.690587,0.406541$
$\mathrm{C}, 2.535818,-0.532592,-0.640626$
$\mathrm{C}, 1.842248,0.699545,-0.094154$
$\mathrm{O}, 1.010708,0.38588,1.038093$
$\mathrm{H}, 3.143047,-0.266542,-1.509613$
H, 3.195008, $-0.970786,0.113574$
H, $1.803834,-1.283989,-0.945673$
H, $1.168435,1.130073,-0.834539$
H, 2.568674, $1.461996,0.195759$
H, $1.561548,-0.017215,1.72571$

Linear-gauche $(\mathrm{EtOH})_{3}$

C, -1.645612, -2.255316, -0.23564

C, $-2.324639,-1.121163,0.505699$

O, $-2.639611,-0.024805,-0.373971$

$\mathrm{H},-3.194994,-0.353442,-1.096795$

$\mathrm{H},-2.319961,-2.687567,-0.979967$

H, $-0.746202,-1.900107,-0.743598$

$\mathrm{H},-1.360628,-3.043617,0.465819$

H, $-3.239911,-1.463772,0.993558$

$\mathrm{H},-1.667364,-0.6989,1.265734$

H, $-0.998508,0.644417,-0.808814$

O, $-0.05968,0.919044,-0.873831$

C, 0.03896, 2.303519, -0.505953

C, $-0.452568,2.552369,0.908254$

$\mathrm{H},-0.523933,2.907381,-1.222741$

H, 1.093946, 2.561458, -0.599698

$\mathrm{H}, 0.115165,1.95394,1.624843$

H, $-1.51169,2.299865,1.001495$

$\mathrm{H},-0.330187,3.607961,1.165513$

$\mathrm{H}, 0.889062,-0.062425,0.335708$

$\mathrm{O}, 1.421585,-0.592676,0.965485$

C, 2.388687, -1.332012, 0.214677
C, 3.383601, $-0.421559,-0.483378$

$\mathrm{H}, 1.881543,-1.972626,-0.514018$

H, 2.898861, $-1.9777,0.930151$

$\mathrm{H}, 3.897389,0.211378,0.244327$

$\mathrm{H}, 2.876997,0.221082,-1.208395$

H, 4.13149, -1.014869, -1.016538

Linear-gauche $(\mathrm{EtOH})_{4}$

C, $-0.275632,-1.883029,-2.080966$

C, $-0.629097,-2.483015,-0.734408$

O, $-2.015048,-2.269478,-0.403168$

$\mathrm{H},-2.565853,-2.656474,-1.100154$

$\mathrm{H},-0.87265,-2.344218,-2.872755$

H, - $0.460087,-0.806004,-2.087735$

$\mathrm{H}, 0.77902,-2.057336,-2.305351$

$\mathrm{H},-0.420455,-3.555017,-0.717173$

$\mathrm{H},-0.065793,-2.00962,0.070243$

$\mathrm{H},-2.182202,-0.491322,-0.18504$

$\mathrm{O},-2.106793,0.476639,-0.040211$

C, $-2.589828,0.78887,1.275896$

C, $-1.830765,0.033568,2.351352$

$\mathrm{H},-3.659266,0.568469,1.327753$

$\mathrm{H},-2.457494,1.864882,1.392525$

$\mathrm{H},-0.76263,0.258259,2.297491$

H, -1.968941, -1.044763, 2.239776

$\mathrm{H},-2.194816,0.323795,3.34052$

$\mathrm{H},-0.402342,0.982366,-0.1066$

O, 0.517551, 1.331569, -0.118674

C, 0.626063, 2.282317, -1.187919

C, $-0.286565,3.474979,-0.970011$

$\mathrm{H}, 0.398722,1.790581,-2.138371$

$\mathrm{H}, 1.671012,2.592791,-1.20847$

$\mathrm{H},-0.051348,3.970438,-0.025069$

$\mathrm{H},-1.333346,3.161644,-0.949417$

H, $-0.16332,4.197937,-1.780624$

$\mathrm{H}, 1.75354,0.041939,-0.415221$

$\mathrm{O}, 2.53232,-0.540773,-0.541374$

C, 3.412354, $-0.322795,0.564883$

C, $2.826239,-0.847147,1.863922$

$\mathrm{H}, 3.638694,0.744892,0.651066$

$\mathrm{H}, 4.339432,-0.844611,0.325123$

H, 2.621606, -1.917954, 1.786746

H, 1.892678, -0.330231, 2.101987 
H, 3.524989, - -0.6866, 2.689672

Linear-gauche $(\mathrm{EtOH})_{5}$

C, -2.380791, 2.099187, -1.384359

C, $-2.557697,0.693752,-1.923553$

O, -3.541426, -0.047074, - 1.176719

$\mathrm{H},-4.386842,0.424285,-1.220163$

$\mathrm{H},-3.312615,2.664803,-1.471215$

$\mathrm{H},-2.081068,2.07667,-0.334205$

$\mathrm{H},-1.61021,2.623018,-1.95558$

$\mathrm{H},-2.849055,0.711156,-2.976076$

$\mathrm{H},-1.635118,0.121722,-1.83096$

H, $-2.912572,-0.344197,0.489529$

O, $-2.468592,-0.531733,1.344818$

C, $-2.079131,-1.914067,1.364161$

C, $-1.170176,-2.261129,0.198993$

H, $-2.974982,-2.540575,1.357699$

H, $-1.56901,-2.064705,2.31562$

H, $-0.312464,-1.585496,0.171223$

$\mathrm{H},-1.707119,-2.184889,-0.749524$

H, $-0.802074,-3.285775,0.301852$

$\mathrm{H},-1.054426,0.535352,1.531046$

O, $-0.289545,1.142601,1.651278$

C, 0.420617, 0.774042, 2.843598

C, $1.340608,-0.41165,2.613634$

H, $-0.300032,0.558618,3.63664$

$\mathrm{H}, 0.9944,1.652183,3.140685$

$\mathrm{H}, 2.059349,-0.191883,1.820024$

H, $0.768508,-1.299679,2.335273$

$\mathrm{H}, 1.896931,-0.637011,3.527843$

$\mathrm{H}, 0.614381,0.999508,0.12517$

$\mathrm{O}, 1.061028,1.015141,-0.751438$

C, 1.611914, 2.326922, -0.937446

C, 2.734625, 2.612247, 0.043904

$\mathrm{H}, 0.816551,3.070773,-0.83696$

$\mathrm{H}, 1.978363,2.357593,-1.963781$

$\mathrm{H}, 3.540431,1.882889,-0.067431$

$\mathrm{H}, 2.366201,2.572812,1.072524$

$\mathrm{H}, 3.14461,3.610393,-0.132619$

H, 2.40799, $-0.210549,-0.860827$

O, 3.148211, $-0.849601,-0.933421$

C, 2.606884, $-2.171323,-0.862001$

C, $1.737599,-2.494892,-2.064644$

$\mathrm{H}, 2.03917,-2.288812,0.066415$

H, 3.461336, -2.847373, -0.817222

H, 2.322027, -2.429464, -2.985737

$\mathrm{H}, 0.899602,-1.796764,-2.13733$

$\mathrm{H}, 1.333603,-3.507814,-1.981782$
Linear-gauche $(\mathrm{EtOH})_{6}$

C, $-3.673282,-1.471875,-1.360683$

C, $-3.711965,-1.6401,0.145022$

O, $-4.431121,-0.569191,0.78548$

$\mathrm{H},-5.33578,-0.550406,0.438786$

$\mathrm{H},-4.683771,-1.50165,-1.777439$

$\mathrm{H},-3.209699,-0.521058,-1.633381$

H, $-3.095498,-2.282998,-1.811266$

H, $-4.171491,-2.591651,0.421555$

$\mathrm{H},-2.708422,-1.606658,0.56745$

$\mathrm{H},-3.56356,1.000592,0.585297$

$\mathrm{O},-3.01658,1.81233,0.505343$

C, $-2.354332,2.042748,1.759225$

C, $-1.535535,0.840476,2.193354$

H, -3.098536, 2.29929, 2.517845

H, $-1.717413,2.913354,1.601933$

$\mathrm{H},-0.8424,0.544743,1.403738$

$\mathrm{H},-2.185143,-0.006818,2.425807$

$\mathrm{H},-0.955058,1.081151,3.0884$

H, - $1.825378,1.603454,-0.795059$

O, $-1.182481,1.492129,-1.531994$

C, $-0.287813,2.615304,-1.549716$

C, $0.849354,2.451283,-0.557258$

$\mathrm{H},-0.855628,3.527146,-1.347412$

$\mathrm{H}, 0.101503,2.679949,-2.566313$

$\mathrm{H}, 1.415091,1.542359,-0.77313$

$\mathrm{H}, 0.473443,2.393599,0.467177$

$\mathrm{H}, 1.531614,3.303527,-0.624649$

H, - $0.483749,-0.096984,-1.170546$

$\mathrm{O},-0.188536,-1.008431,-0.944542$

C, $0.234653,-1.663591,-2.147918$

C, $1.575617,-1.142375,-2.632031$

$\mathrm{H},-0.53071,-1.530375,-2.91729$

H, 0.294545, -2.726482, -1.912681

$\mathrm{H}, 2.344966,-1.290399,-1.869853$

$\mathrm{H}, 1.512479,-0.076658,-2.868136$

$\mathrm{H}, 1.880635,-1.674853,-3.537188$

$\mathrm{H}, 1.042761,-0.803327,0.317782$

O, 1.634305, -0.706449, 1.098397

C, $1.346149,-1.781747,2.001556$

C, $1.779723,-3.12025,1.431713$

$\mathrm{H}, 0.275532,-1.7861,2.228034$

H, 1.886393, -1.557957, 2.921697

H, 2.853866, -3.121793, 1.230399

$\mathrm{H}, 1.250438,-3.329653,0.498033$

$\mathrm{H}, 1.558166,-3.924588,2.138385$

$\mathrm{H}, 3.36924,-0.676573,0.601102$ 
O, 4.30253, -0.606165, 0.30666

C, $4.486755,0.69767,-0.2515$

C, $4.345399,1.788618,0.795833$

H, 3.775476, 0.855625, - 1.068796

H, 5.490747, 0.707185, -0.677239

H, 5.084372, 1.652376, 1.589441

$\mathrm{H}, 3.348209,1.76937,1.243185$

$\mathrm{H}, 4.499484,2.772512,0.34401$

Linear-gauche $(\mathrm{EtOH})_{7}$

H, 5.796198, -1.185187, -1.553721

O, 4.97093, $-0.681452,-1.619698$

C, $3.88172,-1.599784,-1.830865$

C, 3.775187, $-2.615975,-0.711304$

H, 2.987783, $-0.979293,-1.882502$

H, 4.015723, -2.093528, -2.795998

H, 3.634473, $-2.119733,0.251554$

$\mathrm{H}, 4.67959,-3.228688,-0.661169$

H, 2.924148, $-3.278197,-0.889975$

$\mathrm{H}, 4.644697,0.360469,-0.187543$

O, 4.382546, 0.940435, 0.560439

C, 3.941671, 2.201456, 0.032045

C, $2.793915,2.034323,-0.947187$

$\mathrm{H}, 4.786466,2.706716,-0.443569$

$\mathrm{H}, 3.635191,2.792904,0.894925$

$\mathrm{H}, 1.973515,1.481657,-0.484849$

$\mathrm{H}, 3.1217,1.495133,-1.83925$

$\mathrm{H}, 2.419009,3.013455,-1.258208$

H, 3.110056, 0.146574, 1.493374

O, 2.412225, - $-0.302541,2.023515$

C, 1.971239, 0.587641, 3.060542

C, $0.954028,1.595619,2.556539$

$\mathrm{H}, 2.841167,1.094816,3.485934$

$\mathrm{H}, 1.534253,-0.040033,3.837724$

$\mathrm{H}, 0.070998,1.090034,2.158482$

$\mathrm{H}, 1.380404,2.22329,1.770421$

$\mathrm{H}, 0.63601,2.244205,3.377774$

$\mathrm{H}, 1.252768,-0.756918,0.766849$

O, 0.650553, - $1.04395,0.04226$

C, $-0.054028,-2.213514,0.482031$

C, $-0.964768,-1.913879,1.658807$

$\mathrm{H}, 0.669248,-2.990855,0.744009$

$\mathrm{H},-0.628621,-2.561803,-0.376601$

$\mathrm{H},-1.683802,-1.132329,1.402243$

$\mathrm{H},-0.381045,-1.582025,2.521738$

$\mathrm{H},-1.516505,-2.813128,1.945956$

$\mathrm{H},-0.370123,0.34891,-0.340477$

O, $-0.872244,1.132445,-0.663046$
C, $-0.815925,1.122673,-2.096861$

C, $-1.664076,0.010138,-2.685526$

$\mathrm{H}, 0.22638,1.020993,-2.412737$

H, -1.173832, 2.098777, - 2.424273

H, -2.708956, 0.119782, -2.385072

H, $-1.30351,-0.966074,-2.350159$

H, -1.614017, 0.035772, -3.777619

H, $-2.482899,1.048452,0.081496$

$\mathrm{O},-3.352704,1.035473,0.542579$

C, $-3.899651,2.361518,0.510494$

C, $-4.218159,2.806842,-0.905315$

$\mathrm{H},-3.195901,3.052139,0.983437$

$\mathrm{H},-4.80402,2.328504,1.118236$

$\mathrm{H},-4.928643,2.121367,-1.374029$

$\mathrm{H},-3.310381,2.839464,-1.513218$

$\mathrm{H},-4.658027,3.807823,-0.89493$

$\mathrm{H},-4.449449,-0.134803,-0.28662$

$\mathrm{O},-4.963137,-0.842082,-0.731735$

C, $-4.3879,-2.093463,-0.346141$

C, $-4.720196,-2.448949,1.092848$

H, $-3.30308,-2.061517,-0.493992$

H, -4.795866, -2.841944, -1.026618

$\mathrm{H},-5.803252,-2.503466,1.228148$

H, $-4.322318,-1.697319,1.779528$

H, -4.28922, -3.418883, 1.356136

Linear-gauche $(\mathrm{EtOH})_{8}$

H, 5.564105, 3.072418, - 0.469361

$\mathrm{O}, 4.898672,2.603188,0.05586$

C, 3.595507, 2.883823, -0.488083

C, 3.488087, 2.471431, - 1.942463

$\mathrm{H}, 2.897943,2.31921,0.129567$

H, 3.381732, 3.947999, -0.365841

H, 3.706922, 1.407809, -2.061162

$\mathrm{H}, 4.186628,3.044041,-2.558806$

$\mathrm{H}, 2.475805,2.661624,-2.308386$

$\mathrm{H}, 5.188841,0.832916,0.217945$

$\mathrm{O}, 5.275612,-0.135423,0.356965$

C, 5.034546, - $0.420314,1.743611$

C, 3.64913, 0.020348, 2.180597

H, 5.803385, 0.067386, 2.348882

$\mathrm{H}, 5.15366,-1.498123,1.851497$

$\mathrm{H}, 2.883031,-0.424129,1.5412$

H, 3.557346, 1.108022, 2.133645

H, 3.462803, $-0.292823,3.211667$

$\mathrm{H}, 4.156717,-0.957061,-0.745662$

$\mathrm{O}, 3.530023,-1.362479,-1.388214$

C, 3.550661, $-2.788815,-1.232359$ 
C, 2.847334, $-3.237897,0.035848$

$\mathrm{H}, 4.588223,-3.132923,-1.241038$

H, 3.053577, -3.196207, -2.112704

H, 1.810603, $-2.892026,0.047717$

H, 3.354839, $-2.849533,0.922103$

H, 2.846338, $-4.329907,0.094216$

H, 2.020726, - $0.530445,-1.018213$

$\mathrm{O}, 1.218484,0.005663,-0.820537$

C, $0.334321,-0.055863,-1.947599$

C, $-0.171099,-1.465909,-2.193458$

$\mathrm{H}, 0.851357,0.327104,-2.831992$

$\mathrm{H},-0.493669,0.616788,-1.720456$

$\mathrm{H},-0.684917,-1.85217,-1.310092$

$\mathrm{H}, 0.658271,-2.135451,-2.437213$

$\mathrm{H},-0.87155,-1.471347,-3.032831$

$\mathrm{H}, 0.430685,-0.461792,0.672756$

O, $-0.04716,-0.646338,1.51534$

C, $-0.239819,0.59785,2.204093$

C, $-1.018598,1.595966,1.365426$

$\mathrm{H}, 0.733899,1.008703,2.48707$

$\mathrm{H},-0.779523,0.352774,3.11901$

$\mathrm{H},-1.958151,1.156531,1.023303$

$\mathrm{H},-0.439757,1.904121,0.491268$

$\mathrm{H},-1.244904,2.487842,1.95643$

H, $-1.574826,-1.43419,1.098748$

$\mathrm{O},-2.406249,-1.896712,0.84293$

C, $-3.182784,-2.149229,2.023003$

C, $-3.903244,-0.903797,2.507173$

$\mathrm{H},-2.528059,-2.546947,2.802864$

$\mathrm{H},-3.898992,-2.926813,1.756705$

H, -4.566616, -0.516034, 1.729809

H, $-3.189384,-0.123442,2.782145$

H, $-4.50774,-1.139378,3.387595$

H, $-3.131317,-0.804384,-0.356946$

$\mathrm{O},-3.476973,-0.235467,-1.081832$

C, $-4.136262,-1.080727,-2.03483$

C, $-5.363491,-1.750898,-1.443278$

H, $-3.430552,-1.830206,-2.403337$

$\mathrm{H},-4.411718,-0.43513,-2.86925$

H, $-6.075344,-1.004213,-1.083023$

H, $-5.084682,-2.399926,-0.608781$

$\mathrm{H},-5.858299,-2.364085,-2.201381$

H, $-4.667813,1.002465,-0.485163$

$\mathrm{O},-5.324304,1.673942,-0.201662$

C, $-4.622549,2.739206,0.444928$

C, $-3.681971,3.45862,-0.506414$

$\mathrm{H},-4.073436,2.351022,1.308493$

$\mathrm{H},-5.385668,3.423588,0.817143$

H, -4.242004, 3.893897, -1.337763

H, -2.939733, 2.767296, -0.914474

H, -3.15223, 4.261359, 0.014129
Cyclic-gauche $(\mathrm{EtOH})_{3}$

C, $2.378053,-2.515346,0.070234$

C, $0.956936,-2.18314,0.482757$

$\mathrm{O}, 0.201468,-1.6403,-0.610711$

$\mathrm{H}, 0.621136,-0.798204,-0.879189$

$\mathrm{H}, 2.379946,-3.243751,-0.74384$

H, 2.902805, -1.616124, -0.26449

H, 2.927822, $-2.937139,0.915598$

$\mathrm{H}, 0.423092,-3.079872,0.795853$

$\mathrm{H}, 0.952147,-1.474536,1.316801$

H, -1.167596, $-0.563214,0.234677$

O, $-1.51425,0.293015,0.555342$

C, $-2.687028,0.619202,-0.205711$

C, $-3.833451,-0.3255,0.101021$

H, $-2.445075,0.60025,-1.272336$

H, $-2.94298,1.642635,0.067384$

H, $-4.081058,-0.295847,1.16458$

$\mathrm{H},-3.569534,-1.351804,-0.167573$

H, $-4.72059,-0.040173,-0.470438$

H, $-0.006657,1.130769,-0.268875$

O, 0.804299, 1.126327, -0.816646

C, $1.904677,1.574772,-0.013184$

C, $1.755498,3.033514,0.375484$

H, 2.797274, 1.426496, -0.620445

$\mathrm{H}, 1.988261,0.946328,0.878311$

$\mathrm{H}, 0.858213,3.181013,0.98232$

H, 1.681944, 3.661419, -0.515284

$\mathrm{H}, 2.620604,3.358406,0.959465$

Cyclic-gauche $(\mathrm{EtOH})_{4}$

C, 1.775614, 3.500326, -0.970732

C, $0.440444,2.781261,-1.005926$

O, $0.27906,1.920475,0.131428$

$\mathrm{H}, 0.941213,1.194404,0.061272$

$\mathrm{H}, 1.863801,4.098408,-0.060802$

H, 2.599261, 2.78178, -0.998391

$\mathrm{H}, 1.870861,4.163625,-1.834388$

H, $-0.385288,3.491804,-0.965854$

$\mathrm{H}, 0.342135,2.192721,-1.92278$

H, -1.194096, 0.94107, -0.060384

O, $-1.920044,0.278733,-0.130065$

C, $-2.781027,0.440917,1.007031$

C, $-3.501661,1.775183,0.969676$

$\mathrm{H},-2.192364,0.344764,1.92403$

H, $-3.49061,-0.385703,0.968282$ 
$\mathrm{H},-4.099902,1.861142,0.059637$

$\mathrm{H},-2.78408,2.599716,0.995917$

$\mathrm{H},-4.165018,1.871089,1.833215$

$\mathrm{H},-0.94063,-1.194215,0.061572$

O, $-0.278248,-1.920217,0.129833$

C, $-0.441484,-2.77979,-1.008183$

C, $-1.774635,-3.502394,-0.969153$

$\mathrm{H}, 0.386237,-3.488236,-0.97218$

$\mathrm{H},-0.348057,-2.189628,-1.924501$

$\mathrm{H},-2.600239,-2.785943,-0.992412$

$\mathrm{H},-1.857759,-4.102257,-0.059918$

$\mathrm{H},-1.871477,-4.164464,-1.833577$

$\mathrm{H}, 1.194507,-0.940807,-0.062276$

O, 1.920577, $-0.27855,-0.131019$

C, 2.780063, $-0.441011,1.007172$

C, 3.501445, -1.774875, 0.969955

$\mathrm{H}, 2.190046,-0.345786,1.923403$

$\mathrm{H}, 3.489254,0.38602,0.969988$

$\mathrm{H}, 4.101146,-1.859847,0.060784$

$\mathrm{H}, 2.784242,-2.599791,0.994453$

H, 4.163506, -1.871094, 1.834455

Cyclic-gauche $(\mathrm{EtOH})_{5}$

C, 4.21055, 1.594339, - 0.291827

C, 3.347474, 0.509163, 0.324029

O, 2.22699, 0.191995, -0.514859

$\mathrm{H}, 1.602352,0.95309,-0.492105$

$\mathrm{H}, 4.589159,1.273613,-1.265058$

$\mathrm{H}, 3.636741,2.514751,-0.427917$

$\mathrm{H}, 5.062309,1.813876,0.357451$

H, 3.914171, $-0.415476,0.437026$

$\mathrm{H}, 2.988171,0.80914,1.312459$

$\mathrm{H}, 1.388171,-1.2519,0.033023$

$\mathrm{O}, 0.924142,-2.085524,0.282518$

C, 1.240527, -3.082649, -0.701683

C, $2.716012,-3.43395,-0.688867$

$\mathrm{H}, 0.937136,-2.725734,-1.690142$

$\mathrm{H}, 0.6333,-3.95345,-0.453769$

H, 3.019692, $-3.777597,0.302813$

H, 3.323218, - $2.566926,-0.960925$

H, 2.917982, -4.231149, -1.409124

$\mathrm{H},-0.789427,-1.732105,0.077725$

$\mathrm{O},-1.749313,-1.534938,-0.030243$

C, $-2.410677,-1.852602,1.204001$

C, $-2.429472,-3.348244,1.457297$

$\mathrm{H},-3.42465,-1.462036,1.113038$

$\mathrm{H},-1.915445,-1.326535,2.025176$

H, $-1.41179,-3.737136,1.547135$
H, $-2.931962,-3.868945,0.638795$

H, $-2.962244,-3.566764,2.386529$

H, - $1.900673,0.207776,-0.196585$

O, $-2.008245,1.187428,-0.223485$

C, $-2.729639,1.535045,-1.414768$

C, $-4.151036,1.006873,-1.381287$

H, $-2.194971,1.151271,-2.288204$

H, $-2.723189,2.624353,-1.462829$

$\mathrm{H},-4.682116,1.39213,-0.507812$

H, $-4.156965,-0.085536,-1.34295$

H, $-4.688477,1.319275,-2.280601$

H, $-0.410033,1.904588,-0.310291$

O, 0.482694, 2.323413, -0.285264

C, 0.641694, 2.920793, 1.010569

C, $-0.320995,4.076266,1.212852$

$\mathrm{H}, 1.673945,3.267142,1.062093$

$\mathrm{H}, 0.496769,2.159284,1.782595$

$\mathrm{H},-1.356165,3.729852,1.158261$

$\mathrm{H},-0.167823,4.841402,0.448189$

H, $-0.163951,4.530555,2.194775$

Gauche- $[\mathrm{OAc}(\mathrm{EtOH})]^{-}$

$\mathrm{O},-1.115894,-0.588895,1.115468$

$\mathrm{O},-0.917949,0.897652,-0.545687$

C, $-1.565788,0.02416,0.11276$

C, $-2.981533,-0.269015,-0.357101$

$\mathrm{H},-3.419959,-1.10056,0.192856$

H, $-3.595822,0.622943,-0.209891$

H, -2.977921, $-0.493353,-1.425636$

C, 2.314143, $-0.05987,0.442062$

$\mathrm{H}, 1.844764,-0.497944,1.32863$

$\mathrm{H}, 3.356537,0.150551,0.686244$

C, 2.230411, $-1.026931,-0.727092$

$\mathrm{H}, 2.733351,-1.965814,-0.478614$

$\mathrm{H}, 1.188263,-1.251249,-0.969211$

H, 2.709006, -0.600738, -1.612478

$\mathrm{O}, 1.711891,1.200101,0.145264$

$\mathrm{H}, 0.754,1.055233,-0.056035$ 
$\mathrm{H}, 1.620089,2.64625,-0.49325$

H, 0.290844, 3.764762, -0.09994

O, -1.591948, -1.590196, - 0.540433

C, $-2.953373,-1.197211,-0.357414$

H, $-3.196826,-0.37233,-1.034026$

$\mathrm{H},-3.560637,-2.057144,-0.64391$

C, $-3.242154,-0.801291,1.080871$

$\mathrm{H},-4.296042,-0.533384,1.197631$

$\mathrm{H},-3.016978,-1.629792,1.757205$

H, -2.635636, 0.060172, 1.372269

$\mathrm{H},-1.015584,-0.811541,-0.35145$

O, 2.649168, -0.006115, 0.920661

C, $3.188762,-0.735442,-0.183616$

H, 3.020236, -0.177547, -1.111012

$\mathrm{H}, 4.265652,-0.795166,-0.019436$

C, 2.590031, $-2.127787,-0.289624$

$\mathrm{H}, 2.775076,-2.69228,0.627727$

$\mathrm{H}, 1.510221,-2.071766,-0.451178$

H, 3.034817, - $2.671534,-1.127843$

$\mathrm{H}, 1.715164,0.21096,0.689987$

Gauche- $\left[\mathrm{OAc}(\mathrm{EtOH})_{3}\right]^{-}$

$\mathrm{O}, 0.177232,-0.595095,-0.163168$

$\mathrm{O},-1.360801,-1.511038,-1.492811$

C, $-0.319391,-1.585418,-0.788593$

C, $0.409041,-2.910289,-0.697188$

$\mathrm{H}, 1.239221,-2.893997,-1.409415$

H, 0.823429, -3.053987, 0.301338

$\mathrm{H},-0.251952,-3.73731,-0.953223$

O, $-0.008918,1.856479,-1.462005$

C, $-0.324451,2.877143,-0.512673$

$\mathrm{H},-0.386144,3.808291,-1.077626$

$\mathrm{H},-1.308846,2.685895,-0.074879$

C, 0.729268, 2.988153, 0.576354

$\mathrm{H}, 0.461011,3.77814,1.283594$

$\mathrm{H}, 0.816621,2.048709,1.129111$

$\mathrm{H}, 1.703537,3.22715,0.143164$

$\mathrm{O}, 2.361505,-0.82585,1.475699$

C, 3.492508, $-0.877338,0.60378$

H, 4.35426, $-1.107495,1.23183$

H, 3.371262, -1.697093, -0.112319

C, 3.706883, 0.435647, -0.129969

$\mathrm{H}, 2.847311,0.669345,-0.763353$

$\mathrm{H}, 3.846082,1.252106,0.583019$

$\mathrm{H}, 4.594853,0.374413,-0.765625$

H, 1.555592, $-0.755005,0.913528$

H, -0.012136, 0.990709, -0.997224

O, $-3.057471,0.425232,-0.409072$
C, $-2.908159,0.385504,1.011567$

$\mathrm{H},-1.890431,0.676979,1.289057$

$\mathrm{H},-3.592646,1.136826,1.408232$

C, $-3.235049,-0.982923,1.585658$

$\mathrm{H},-2.545319,-1.739301,1.201857$

H, -3.150606, $-0.965442,2.675805$

H, $-4.254102,-1.275414,1.320277$

H, $-2.417257,-0.204219,-0.809911$

Gauche- $\left[\mathrm{OAc}(\mathrm{EtOH})_{4}\right]^{-}$

$\mathrm{O}, 1.059878,-0.312539,0.463836$

O, $-1.065132,-0.570686,-0.150371$

C, $-0.041167,-0.933308,0.501007$

C, $-0.157886,-2.174718,1.35858$

$\mathrm{H},-0.374322,-3.032946,0.717815$

H, 0.756672, -2.360646, 1.919377

H, $-0.995075,-2.059297,2.050639$

O, 1.435536, 1.630201, -1.470625

C, $2.009092,2.695692,-0.71079$

H, 1.986114, 3.578723, - 1.350944

$\mathrm{H}, 1.385653,2.900377,0.165842$

C, 3.434577, 2.38616, -0.285294

$\mathrm{H}, 3.861449,3.233617,0.25883$

H, 3.462485, 1.511165, 0.369233

H, 4.059122, 2.186289, -1.159654

$\mathrm{O}, 3.41798,-1.551918,1.163457$

C, $3.645308,-2.34843,-0.002223$

$\mathrm{H}, 4.456253,-3.034119,0.247027$

$\mathrm{H}, 2.753568,-2.946769,-0.218971$

C, 4.018756, -1.501599, -1.206851

H, 3.206033, $-0.819864,-1.469222$

H, 4.913713, $-0.910772,-0.995749$

$\mathrm{H}, 4.221531,-2.141035,-2.070774$

H, 2.579994, $-1.061149,1.015205$

$\mathrm{H}, 1.247426,0.89605,-0.845051$

O, $-1.658737,2.127571,-0.334564$

C, $-2.447239,2.209272,0.855022$

$\mathrm{H},-3.020206,3.134681,0.78058$

$\mathrm{H},-3.156555,1.375795,0.888153$

C, $-1.585155,2.219938,2.105466$

H, $-0.893353,3.065576,2.085956$

H, -2.210361, 2.304413, 2.998927

H, -1.002992, 1.297402, 2.180813

$\mathrm{H},-1.305221,1.213634,-0.38164$

O, $-3.482883,-1.829881,0.243571$

H, $-2.609842,-1.385745,0.150564$

C, $-4.117213,-1.811785,-1.037898$

H, $-4.992028,-2.458096,-0.955329$ 
$\mathrm{H},-3.447226,-2.246531,-1.786734$

C, $-4.530363,-0.409602,-1.450875$

$\mathrm{H},-3.657184,0.242628,-1.533854$

H, $-5.032164,-0.433625,-2.422335$

$\mathrm{H},-5.218874,0.018533,-0.718007$

Gauche- $\left[\mathrm{OAc}(\mathrm{EtOH})_{5}\right]^{-}$

O, 1.581333, -0.428924, - 0.035713

O, $-0.460108,-0.154748,-0.881959$

C, $0.421232,-0.857389,-0.306121$

C, $0.09308,-2.28141,0.089346$

H, 0.803228, -2.958821, -0.390424

$\mathrm{H}, 0.212484,-2.389272,1.170005$

H, $-0.921295,-2.556949,-0.194116$

O, 2.640281, 1.805257, - 1.287868

C, $3.111362,2.636404,-0.224265$

$\mathrm{H}, 3.437989,3.568737,-0.687035$

$\mathrm{H}, 2.28541,2.869271,0.454652$

C, 4.257481, 1.994991, 0.540109

$\mathrm{H}, 4.608161,2.667312,1.328421$

H, 3.937098, 1.059402, 1.006051

H, 5.093689, 1.781381, - 0.130297

O, 3.439697, -2.134676, 1.078661

C, 4.129994, - $2.690555,-0.042936$

H, 4.753631, $-3.494903,0.349464$

H, 3.409395, $-3.13345,-0.738621$

C, 4.984349, - $1.657406,-0.757529$

H, 4.36299, $-0.854568,-1.162239$

H, 5.712103, -1.221329, -0.068603

$\mathrm{H}, 5.52587,-2.12245,-1.586252$

H, 2.763645, -1.510437, 0.731552

H, 2.206221, 1.0215, -0.884263

O, $-0.589096,2.552465,-0.261046$

C, $-1.332784,2.466885,0.956875$

$\mathrm{H},-1.760428,3.456355,1.125588$

$\mathrm{H},-2.160314,1.760015,0.835695$

C, $-0.456924,2.063347,2.131091$

$\mathrm{H}, 0.348727,2.788382,2.270561$

H, -1.047584, 2.02359, 3.050647

$\mathrm{H},-0.011721,1.078945,1.963987$

$\mathrm{H},-0.396504,1.635672,-0.551243$

O, - $2.931239,-1.149214,-1.499658$

$\mathrm{H},-2.039718,-0.802492,-1.256698$

C, $-3.50118,-0.29346,-2.498082$

$\mathrm{H},-4.417809,-0.7835,-2.827583$

H, $-2.820952,-0.234064,-3.35241$

C, $-3.798905,1.092309,-1.95487$

$\mathrm{H},-2.879714,1.586075,-1.630693$
H, $-4.262028,1.708748,-2.730457$

H, $-4.485035,1.032493,-1.106045$

$\mathrm{O},-4.591342,-1.535186,0.694932$

H, $-3.992078,-1.375828,-0.066624$

C, $-4.204224,-0.667093,1.762966$

$\mathrm{H},-4.911975,-0.84967,2.572385$

H, $-4.313224,0.377369,1.451995$

C, $-2.783231,-0.934303,2.228514$

$\mathrm{H},-2.671414,-1.979351,2.52921$

$\mathrm{H},-2.068151,-0.72317,1.430114$

H, - $2.538394,-0.298694,3.083889$

Gauche- $\left[\mathrm{OAc}(\mathrm{EtOH})_{6}\right]^{-}$

$\mathrm{O}, 0.652543,0.157958,-0.985342$

O, $-1.552813,0.43329,-1.11586$

C, $-0.516445,-0.28971,-1.17807$

C, $-0.656666,-1.763679,-1.492796$

$\mathrm{H},-0.047013,-2.004376,-2.366509$

H, $-0.27365,-2.347339,-0.652478$

$\mathrm{H},-1.692685,-2.039008,-1.683454$

O, 1.314853, 2.858345, -1.090888

C, $1.845073,3.232795,0.1833$

$\mathrm{H}, 2.128668,4.282677,0.098151$

$\mathrm{H}, 1.063925,3.156025,0.944923$

C, 3.045511, 2.386912, 0.572361

$\mathrm{H}, 3.428224,2.69928,1.54851$

$\mathrm{H}, 2.767096,1.331968,0.638134$

H, 3.845573, 2.494449, - 0.164093

O, 2.750659, -1.570587, -0.785446

C, 3.461637, $-1.496023,-2.026662$

H, 4.224404, -2.274441, -1.990522

H, 2.778599, -1.732753, -2.8481

C, 4.090991, $-0.130857,-2.239322$

H, 3.322745, 0.645934, -2.269628

$\mathrm{H}, 4.790724,0.102113,-1.433065$

$\mathrm{H}, 4.6365,-0.110144,-3.186935$

$\mathrm{H}, 1.979472,-0.955694,-0.837164$

$\mathrm{H}, 1.00563,1.927965,-1.032378$

O, -1.645422, 2.416266, 0.833367

C, $-1.944813,1.601147,1.96908$

$\mathrm{H},-2.366602,2.267483,2.723021$

H, $-2.712795,0.867077,1.703743$

C, $-0.707376,0.905018,2.510681$

$\mathrm{H}, 0.043474,1.640753,2.809697$

H, -0.961446, 0.298677, 3.384646

$\mathrm{H},-0.266148,0.25097,1.753993$

$\mathrm{H},-1.494697,1.815411,0.073199$

$\mathrm{O},-4.065027,-0.587379,-1.461464$ 
$\mathrm{H},-3.148725,-0.250006,-1.319635$

C, $-4.929973,0.52913,-1.706391$

H, $-5.880365,0.109232,-2.036875$

H, $-4.52039,1.127877,-2.524632$

C, $-5.121314,1.379698,-0.463612$

$\mathrm{H},-4.169052,1.800855,-0.133024$

$\mathrm{H},-5.804472,2.207293,-0.67429$

$\mathrm{H},-5.543491,0.782285,0.348662$

$\mathrm{O},-4.938301,-2.307313,0.533703$

H, $-4.617255,-1.68208,-0.152237$

C, $-4.266551,-2.016255,1.761397$

H, $-4.689531,-2.692517,2.505118$

H, $-4.4919,-0.991543,2.075444$

C, $-2.764675,-2.218322,1.653343$

$\mathrm{H},-2.539735,-3.238318,1.331046$

H, $-2.330479,-1.521634,0.932424$

H, -2.288041, -2.048334, 2.622728

O, 3.958988, -1.181835, 1.705212

H, 3.575624, $-1.309422,0.811228$

C, 5.268634, $-0.628142,1.562457$

H, 5.221146, 0.30013, 0.984384

H, 5.602957, $-0.375935,2.569545$

C, 6.229569, -1.610267, 0.915194

$\mathrm{H}, 5.888676,-1.881383,-0.087734$

H, 7.226792, -1.169769, 0.829486

$\mathrm{H}, 6.30279,-2.522392,1.512649$

\section{Trans- $[\mathrm{OAc}(\mathrm{EtOH})]^{-}$}

$\mathrm{O}, 1.891036,1.33454,0.169822$

O, $0.9545,-0.637419,-0.321043$

C, $1.949488,0.090665,-0.008924$

C, 3.285281, $-0.616485,0.148811$

H, 4.076795, 0.081207, 0.418862

$\mathrm{H}, 3.199941,-1.387381,0.918153$

H, 3.543961, - $1.115347,-0.788079$

C, $-2.289041,-0.47344,0.168563$

H, $-1.961022,-0.693386,1.189985$

$\mathrm{H},-2.178282,-1.387115,-0.424971$

C, $-3.734616,-0.021703,0.169047$

H, $-4.376528,-0.797756,0.592967$

$\mathrm{H},-3.850303,0.886554,0.765579$

$\mathrm{H},-4.0707,0.186577,-0.849708$

O, $-1.483206,0.566774,-0.386171$

$\mathrm{H},-0.549175,0.241264,-0.388625$
Trans- $\left[\mathrm{OAc}(\mathrm{EtOH})_{2}\right]^{-}$

O, $-0.076991,0.811402,-0.216512$

$\mathrm{O}, 1.472871,2.420805,-0.174045$

C, 0.290397, 2.025226, - 0.056548

C, $-0.80356,3.016211,0.296792$

H, $-1.573125,2.999399,-0.478733$

$\mathrm{H},-1.276131,2.714922,1.234734$

$\mathrm{H},-0.407919,4.025728,0.396633$

O, 1.873766, -1.032191, - 0.736927

C, 2.705911, $-1.034101,0.424225$

H, 3.122315, $-0.034536,0.583764$

H, 2.112687, $-1.298067,1.306367$

C, 3.820847, $-2.038382,0.221968$

H, 4.474665, -2.060947, 1.097258

H, 3.411497, $-3.040326,0.070488$

$\mathrm{H}, 4.421555,-1.772346,-0.651307$

$\mathrm{H}, 1.1763,-0.346615,-0.603733$

O, $-2.665771,0.00503,0.016306$

C, $-2.49536,-1.414388,0.019627$

$\mathrm{H},-1.950511,-1.724149,-0.878$

H, $-1.906926,-1.715148,0.892875$

C, $-3.860859,-2.068735,0.056503$

H, $-4.407184,-1.765083,0.952952$

$\mathrm{H},-4.446185,-1.783631,-0.821248$

$\mathrm{H},-3.760272,-3.156719,0.065746$

$\mathrm{H},-1.766025,0.402158,-0.053783$

Trans- $\left[\mathrm{OAc}(\mathrm{EtOH})_{3}\right]^{-}$

O, 0.009447, 1.01477, 0.145983

O, 1.522413, 2.308794, - -0.863451

C, $0.416138,2.139593,-0.287283$

C, $-0.500692,3.328586,-0.082005$

H, $-1.441501,3.155049,-0.61033$

$\mathrm{H},-0.732581,3.42815,0.980703$

H, - $0.045026,4.248117,-0.445348$

O, $-0.138505,-1.417301,-1.155543$

C, $-0.434661,-2.245851,-0.027891$

$\mathrm{H}, 0.492321,-2.50817,0.493479$

H, -1.073647, -1.701007, 0.673329

C, $-1.133505,-3.49859,-0.513631$

H, -1.349086, -4.161836, 0.327541

$\mathrm{H},-2.075848,-3.246206,-1.00664$

H, $-0.502812,-4.038031,-1.224712$

O, $-2.526563,0.678786,1.118233$

C, $-3.277939,0.325676,-0.046025$ 
$\mathrm{H},-3.535085,1.2285,-0.610571$

H, $-2.674353,-0.316341,-0.694259$

C, $-4.536699,-0.397722,0.38569$

H, $-4.284543,-1.317099,0.920492$

H, $-5.136148,0.235297,1.044957$

H, $-5.143263,-0.657919,-0.485205$

$\mathrm{H},-1.604854,0.875178,0.831698$

$\mathrm{H}, 0.0598,-0.520176,-0.813742$

O, 3.569722, 0.650546, 0.020489

C, 3.123167, $-0.668211,0.345139$

$\mathrm{H}, 2.372139,-0.622613,1.139609$

H, 2.664228, - $1.131398,-0.533333$

C, 4.319301, $-1.476973,0.801023$

H, 4.776326, $-1.022582,1.683626$

H, 4.014341, - $2.4949,1.05607$

H, 5.070069, $-1.529503,0.008768$

$\mathrm{H}, 2.806751,1.17368,-0.311919$

\section{Trans- $\left[\mathrm{OAc}(\mathrm{EtOH})_{4}\right]^{-}$}

O, 0.484046, - $0.718852,-0.160561$

$\mathrm{O},-1.528249,-0.479526,-1.085743$

C, $-0.653749,-1.167761,-0.479372$

C, $-1.008113,-2.585276,-0.086508$

$\mathrm{H},-1.633268,-3.049604,-0.849293$

H, $-0.112151,-3.181886,0.082349$

$\mathrm{H},-1.582192,-2.548827,0.844219$

O, 2.202756, 1.078882, - 1.374112

C, $2.939241,1.568814,-0.250089$

H, 2.47193, 2.484405, 0.127933

$\mathrm{H}, 2.923907,0.826004,0.552593$

C, 4.363246, 1.853098, -0.682076

$\mathrm{H}, 4.941652,2.248513,0.156634$

H, 4.847669, 0.940966, - 1.039742

$\mathrm{H}, 4.376299,2.591828,-1.487549$

O, $-4.156355,-1.262139,-0.811883$

H, $-3.21421,-1.048859,-0.992752$

C, $-4.612584,-0.361429,0.201354$

$\mathrm{H},-3.952084,-0.419113,1.072639$

H, $-4.588281,0.665708,-0.176104$

C, $-6.02574,-0.745667,0.585997$

H, $-6.052456,-1.765343,0.977928$

H, $-6.407567,-0.069037,1.354208$

$\mathrm{H},-6.686845,-0.689407,-0.282374$

O, 2.513814, -1.950553, 1.204165

C, 3.514855, -2.109524, 0.194265

H, 3.409855, $-3.092655,-0.276865$

H, 3.379161, - $1.350492,-0.581086$

C, $4.883942,-1.97605,0.828836$
H, 5.005673, $-0.985275,1.274444$

H, 5.018329, -2.726464, 1.612097

H, 5.666079, $-2.117973,0.078703$

H, 1.704124, -1.599985, 0.772046

$\mathrm{H}, 1.462236,0.533775,-1.035567$

$\mathrm{O},-1.861242,1.994758,0.186989$

C, $-0.584499,2.610227,0.386279$

$\mathrm{H}, 0.03591,1.976227,1.02691$

H, $-0.076874,2.726174,-0.575731$

C, $-0.799387,3.961717,1.034136$

$\mathrm{H},-1.303461,3.850195,1.99724$

$\mathrm{H}, 0.160352,4.456427,1.202631$

H, -1.410541, 4.601926, 0.393238

H, - $1.724684,1.153328,-0.296528$

Trans- $\left[\mathrm{OAc}(\mathrm{EtOH})_{5}\right]^{-}$

O, - $0.86362,-0.234995,0.184606$

O, $1.187768,-1.096996,0.06227$

C, $-0.032894,-1.077195,-0.267237$

C, $-0.520322,-2.138865,-1.229467$

$\mathrm{H},-0.602029,-3.083268,-0.68411$

$\mathrm{H},-1.495257,-1.886238,-1.644593$

H, $0.203865,-2.280108,-2.033157$

$\mathrm{O}, 0.115568,2.336159,0.408172$

C, $0.789733,2.415861,-0.850035$

H, 1.615532, 1.697264, -0.876206

$\mathrm{H}, 0.097055,2.163052,-1.660251$

C, 1.313996, 3.825236, -1.028127

$\mathrm{H}, 1.840779,3.91601,-1.98102$

$\mathrm{H}, 0.492095,4.545514,-1.017527$

H, 2.008758, 4.080027, -0.223892

O, $-4.828423,-1.272047,0.136748$

$\mathrm{H},-4.280591,-0.69037,-0.431918$

C, $-4.032564,-1.649947,1.265563$

$\mathrm{H},-3.155902,-2.212937,0.930149$

$\mathrm{H},-3.681742,-0.7542,1.787649$

C, $-4.881523,-2.499492,2.186995$

$\mathrm{H},-5.22374,-3.399335,1.670097$

$\mathrm{H},-4.303266,-2.803518,3.06258$

$\mathrm{H},-5.755885,-1.939172,2.526964$

O, $-3.093495,0.440594,-1.222549$

C, $-3.427789,1.760607,-0.770334$

$\mathrm{H},-3.504846,1.764712,0.321366$

$\mathrm{H},-2.634446,2.455473,-1.059673$

C, $-4.743831,2.16309,-1.40046$

H, $-4.664168,2.156315,-2.490186$

H, -5.538546, 1.474058, -1.104025

H, -5.023044, 3.169861, -1.081085 
$\mathrm{H},-2.268936,0.157408,-0.760121$

$\mathrm{H},-0.224181,1.420245,0.49689$

O, 2.548264, 0.068875, 2.177907

C, $3.313419,1.187536,1.722694$

$\mathrm{H}, 3.8486,0.92405,0.805028$

H, 2.649028, 2.027749, 1.502284

C, 4.294888, 1.570036, 2.810726

$\mathrm{H}, 4.968438,0.738292,3.031245$

$\mathrm{H}, 4.895085,2.427287,2.496301$

H, 3.763452, 1.839876, 3.726596

$\mathrm{H}, 1.990293,-0.259718,1.440851$

O, 3.146502, $-2.508843,-1.237271$

$\mathrm{H}, 2.359926,-2.124276,-0.791192$

C, $4.218965,-1.579767,-1.060579$

H, 3.922426, -0.596336, -1.440067

$\mathrm{H}, 4.448607,-1.478661,0.0053$

C, 5.431035, - $2.088177,-1.812774$

H, 5.211308, $-2.175486,-2.879556$

H, 6.270122, $-1.399578,-1.687743$

H, 5.732012, -3.069493, -1.437839

Trans- $\left[\mathrm{OAc}(\mathrm{EtOH})_{6}\right]^{-}$

O, -1.003886, -0.343632, -1.201943

O, $0.829629,0.884235,-0.888067$

C, $-0.405356,0.67007,-0.730378$

C, $-1.220154,1.656658,0.078058$

$\mathrm{H},-2.128781,1.918432,-0.467114$

H, $-1.517397,1.178987,1.015898$

$\mathrm{H},-0.648257,2.556158,0.30147$

$\mathrm{O},-0.219018,-2.756856,-0.044489$

C, $-0.060786,-2.241223,1.280807$

$\mathrm{H}, 0.641517,-1.401409,1.270509$

H, -1.023361, - $1.875729,1.654239$

C, $0.460228,-3.351247,2.169196$

$\mathrm{H}, 0.580315,-2.991201,3.193633$

H, -0.238626, -4.191306, 2.179997

H, 1.428535, -3.709007, 1.810439

$\mathrm{O},-5.050674,1.35346,-0.657169$

H, $-4.484075,0.562566,-0.527403$

C, $-5.253551,1.955322,0.624205$

$\mathrm{H},-5.694315,1.225089,1.310733$

H, $-4.29275,2.274614,1.04108$

C, $-6.174804,3.144825,0.456997$

$\mathrm{H},-7.141506,2.827484,0.058479$

$\mathrm{H},-6.340803,3.634129,1.419657$

H, -5.737287, 3.87362, - 0.2297

O, $-3.445705,-0.835158,-0.073767$

C, $-3.957453,-2.067726,-0.598381$
H, $-4.044749,-1.993963,-1.68627$

$\mathrm{H},-3.262561,-2.878474,-0.363069$

C, $-5.310579,-2.329415,0.027743$

H, $-5.222347,-2.394943,1.114915$

H, $-6.01044,-1.526927,-0.21832$

$\mathrm{H},-5.721538,-3.271332,-0.34285$

$\mathrm{H},-2.572544,-0.648647,-0.495279$

$\mathrm{H},-0.444982,-2.000048,-0.621198$

$\mathrm{O}, 2.794644,-0.588293,-2.162749$

C, 2.748413, $-1.960598,-1.764955$

$\mathrm{H}, 2.593013,-2.031052,-0.684151$

H, 1.910725, -2.462525, -2.259532

C, $4.05649,-2.618463,-2.151841$

H, 4.893916, -2.140891, -1.636753

H, 4.042189, $-3.677295,-1.88227$

H, 4.220546, -2.540056, -3.229485

$\mathrm{H}, 2.025106,-0.122393,-1.770042$

$\mathrm{O}, 2.3607,2.815654,0.284042$

$\mathrm{H}, 1.713519,2.165725,-0.077635$

C, 3.368755, 3.021189, -0.717

$\mathrm{H}, 3.833559,2.063762,-0.970473$

H, 2.903851, 3.425213, - 1.620592

C, $4.400165,3.984103,-0.170415$

$\mathrm{H}, 4.872066,3.57439,0.726187$

H, 5.17769, 4.165511, - 0.916096

H, 3.936327, 4.939716, 0.085144

$\mathrm{O}, 3.556603,1.120186,2.160587$

H, 3.158463, 1.820466, 1.602463

C, 3.607445, $-0.072456,1.368976$

$\mathrm{H}, 2.649464,-0.214879,0.864028$

$\mathrm{H}, 4.384499,0.023845,0.603439$

C, 3.910233, -1.245572, 2.276902

H, 3.123612, $-1.368078,3.025569$

H, 3.984225, -2.167471, 1.694536

H, 4.860549, - $1.091644,2.794776$

$\left[\mathrm{Zn}(\mathrm{EtOH})_{4}\right]^{2+}(\mathrm{EtOH})$

Zn, 0.459271, 0.401758, -0.315951

$\mathrm{O},-1.205625,1.48226,-0.673547$

$\mathrm{O}, 0.093321,-0.52379,1.501464$

O, 2.497443, 0.755481, -0.117096

H, 2.860288, 1.176026, -0.914092

O, 0.405082, -1.07739, -1.73073

$\mathrm{H},-0.875945,-0.41198,1.63049$

$\mathrm{H},-0.050838,-0.79661,-2.540732$

H, $-1.865556,1.101684,-0.047633$

C, $-1.159091,2.925206,-0.503071$

$\mathrm{H},-0.355528,3.267854,-1.151693$ 
H, $-0.899521,3.144385,0.533475$

C, $2.931218,1.499821,1.056425$

H, 2.453885, 2.480171, 1.041652

H, 2.557313, 0.930796, 1.904279

C, $1.570905,-1.87546,-2.07692$

$\mathrm{H}, 2.234285,-1.26901,-2.693746$

$\mathrm{H}, 2.05956,-2.08951,-1.129328$

C, $0.448008,-1.9278,1.586408$

$\mathrm{H}, 1.50905,-1.97223,1.346785$

H, $-0.110989,-2.48258,0.83096$

C, $0.177431,-2.45484,2.978092$

$\mathrm{H}, 0.471437,-3.50529,3.037274$

$\mathrm{H}, 0.747126,-1.89193,3.720208$

H, - $0.885907,-2.38422,3.220548$

C, 1.147371, $-3.14358,-2.782485$

$\mathrm{H}, 0.623605,-2.91435,-3.713688$

$\mathrm{H}, 2.031471,-3.73768,-3.025158$

H, 0.492669, -3.74016, -2.143948

C, 4.438216, 1.612943, 1.07755

H, 4.797255, 2.158617, 0.201302

$\mathrm{H}, 4.750083,2.159505,1.97033$

H, 4.900314, 0.624166, 1.0948

C, $-2.486383,3.53848,-0.886914$

$\mathrm{H},-2.436343,4.623235,-0.767365$

$\mathrm{H},-2.72859,3.313142,-1.927278$

H, -3.287194, 3.160809, -0.246399

$\mathrm{O},-2.578883,-0.02182,1.164435$

C, $-3.109399,-1.20926,0.529765$

$\mathrm{H},-2.40037,-1.44543,-0.263772$

$\mathrm{H},-3.109559,-2.02337,1.256687$

C, $-4.49602,-0.96406,-0.024152$

H, $-4.482605,-0.15442,-0.757152$

H, $-4.864183,-1.86971,-0.511813$

H, $-5.189946,-0.70295,0.778776$

H, $-3.187634,0.271647,1.860369$

$\left[\mathrm{Zn}(\mathrm{EtOH})_{4}\right]^{2+}(\mathrm{EtOH})_{2}$

Zn, 0.030411, 0.185417, -0.11279

O, $1.547705,1.29523,0.732181$

O, 1.137306, -0.973197, -1.416988

O, -1.420637, 1.626448, -0.407934

H, $-2.310542,1.292595,-0.15324$

O, -1.221556, -1.171549, 0.804187

H, 2.080186, - $0.712933,-1.293319$

$\mathrm{H},-2.135561,-0.814944,0.874256$

$\mathrm{H}, 2.37388,1.113378,0.228872$

C, 1.430802, 2.705029, 1.040801

$\mathrm{H}, 0.470989,2.818378,1.539918$
H, 1.415243, 3.270957, 0.107948

C, $-1.497808,2.496897,-1.560076$

H, $-0.4676,2.691435,-1.852714$

H, $-1.998745,1.963905,-2.369568$

C, $-0.799304,-1.755755,2.062302$

$\mathrm{H}, 0.281571,-1.866061,1.987354$

H, -1.02494, -1.053701, 2.865829

C, $0.998151,-2.416261,-1.388529$

$\mathrm{H},-0.047761,-2.611503,-1.615531$

$\mathrm{H}, 1.214281,-2.775078,-0.38108$

C, $1.914528,-3.048339,-2.411659$

H, 1.785405, -4.132941, -2.399778

$\mathrm{H}, 1.683633,-2.681567,-3.41378$

$\mathrm{H}, 2.96129,-2.826732,-2.18706$

C, $-1.475554,-3.090691,2.278163$

H, -1.240733, -3.775801, 1.460571

$\mathrm{H},-1.126304,-3.532939,3.214235$

$\mathrm{H},-2.559703,-2.971113,2.338705$

C, $-2.221489,3.775576,-1.200681$

$\mathrm{H},-1.703916,4.29556,-0.391581$

H, $-2.261134,4.436302,-2.069959$

H, $-3.246292,3.563523,-0.885807$

C, 2.575166, 3.132409, 1.932635

$\mathrm{H}, 2.478955,4.19328,2.175233$

$\mathrm{H}, 2.5717,2.560582,2.862877$

$\mathrm{H}, 3.534069,2.982993,1.429613$

$\mathrm{O}, 3.536394,0.230587,-0.836583$

C, $4.609698,-0.459226,-0.152128$

H, 5.138116, -1.084394, - 0.872919

$\mathrm{H}, 5.300185,0.283787,0.24918$

C, 3.998946, -1.291779, 0.951269

H, 3.294849, $-2.021109,0.542976$

H, 4.786695, -1.834836, 1.47761

H, 3.477628, $-0.659319,1.673458$

H, 3.90145, 0.753382, -1.566956

O, $-3.57746,0.212237,0.490225$

$\mathrm{H},-4.127511,0.542258,1.217549$

C, $-4.425786,-0.334606,-0.545892$

H, $-5.104313,0.447529,-0.889626$

C, $-5.181848,-1.549434,-0.052663$

$\mathrm{H},-5.802614,-1.95057,-0.857403$

H, $-4.489125,-2.329194,0.271506$

$\mathrm{H},-5.834667,-1.286667,0.783353$

H, $-3.748029,-0.591922,-1.358972$

$\left[\mathrm{Zn}(\mathrm{EtOH})_{5}\right]^{2+}(\mathrm{EtOH})$

Zn, - $0.453142,0.226843,-0.094591$

O, -1.721504, 0.288639, 1.617856

O, - $0.499984,2.077529,-1.034893$ 
O, - $2.177903,-0.45905,-1.166199$

$\mathrm{O}, 0.43965,-1.544595,-0.681532$

$\mathrm{O}, 1.279858,0.759802,0.950467$

$\mathrm{H},-1.347883,0.815593,2.342123$

H, $-1.935159,-0.675528,-2.081107$

C, 0.511534, 3.118465, -1.033791

C, $-2.041712,-1.038817,2.117285$

C, $-3.422349,0.2831,-1.169399$

C, $-0.14495,-2.852397,-0.447455$

C, $1.309688,1.663202,2.075622$

H, -3.283194, 1.204966, -1.736825

H, -1.179854, -2.777207, -0.769605

$\mathrm{H}, 0.53554,2.403957,1.88089$

$\mathrm{H},-1.123036,-1.507873,2.472176$

$\mathrm{H},-3.60738,0.531736,-0.127204$

$\mathrm{H}, 1.047615,1.109693,2.979897$

C, $-4.54072,-0.556827,-1.745797$

$\mathrm{H},-5.474666,0.00947,-1.719694$

$\mathrm{H},-4.330652,-0.821319,-2.785196$

H, $-4.67212,-1.472954,-1.166246$

C, 2.6726, 2.308112, 2.202816

$\mathrm{H}, 2.67714,3.002332,3.046451$

$\mathrm{H}, 3.441576,1.551721,2.378911$

H, 2.924963, 2.86154, 1.295796

C, $0.41841,3.94189,-2.299074$

H, $-0.553116,4.437515,-2.372062$

H, 1.193265, 4.712592, -2.289148

C, $0.603876,-3.907808,-1.231272$

$\mathrm{H}, 1.645723,-3.967128,-0.906508$

$\mathrm{H}, 0.139673,-4.883438,-1.067233$

C, $-3.087623,-0.966581,3.207227$

$\mathrm{H},-3.337663,-1.977293,3.538598$

H, $-2.71684,-0.406113,4.069126$

H, $-3.996973,-0.488822,2.837369$

H, $-2.40989,-1.582729,1.249863$

$\mathrm{H}, 0.578227,-3.685863,-2.300138$

$\mathrm{H}, 0.563336,3.314022,-3.180676$

H, 1.376001, $-1.567919,-0.381967$

$\mathrm{H}, 0.376227,3.732878,-0.143239$

$\mathrm{H}, 1.460478,2.593512,-0.965058$

H, $-0.118968,-3.060808,0.623075$

$\mathrm{H},-1.381722,2.479172,-1.081864$

H, 1.941479, 0.042649, 1.055459

O, 2.972517, -1.309051, 0.437269

H, 3.332459, -1.972946, 1.045478

C, 4.04696, -0.726291, -0.335026

$\mathrm{H}, 3.571351,0.0378,-0.948631$

$\mathrm{H}, 4.745541,-0.24076,0.348311$

C, $4.74179,-1.765593,-1.187477$

H, 5.537663, -1.293011, -1.768132

H, 5.191001, -2.540626, -0.561621
H, 4.03801, -2.233037, -1.879347

$\left[\mathrm{Zn}(\mathrm{EtOH})_{5}\right]^{2+}(\mathrm{EtOH})_{2}$

Zn, -0.096503, -0.174137, -0.099109

$\mathrm{O},-1.231932,-1.923241,-0.569981$

O, $-1.612137,1.053895,-0.778196$

$\mathrm{O}, 1.12903,1.554274,-0.001378$

$\mathrm{O}, 1.620078,-1.233428,-0.610832$

O, $-0.488092,-0.579835,1.909121$

H, -2.161026, -1.678587, -0.369929

H, $2.075955,1.447698,-0.231689$

C, $-1.525071,1.761987,-2.036875$

C, $-1.185002,-2.418355,-1.929485$

C, $0.861621,2.872369,0.518415$

C, $1.986431,-2.503975,-0.027602$

C, $0.081323,0.312713,2.902919$

$\mathrm{H}, 1.288472,2.946273,1.520479$

H, 2.610427, $-3.034758,-0.748375$

$\mathrm{H}, 1.161512,0.358938,2.757499$

$\mathrm{H},-0.132224,-2.58831,-2.145218$

H, $-0.221598,2.948151,0.591696$

$\mathrm{H},-0.350884,1.286687,2.684442$

C, 1.429602, 3.940746, - 0.392282

$\mathrm{H}, 1.205756,4.9289,0.016557$

H, 2.515801, 3.844355, -0.470993

H, 0.996669, 3.871817, -1.392329

C, $-0.272588,-0.149723,4.297717$

$\mathrm{H}, 0.143966,0.546529,5.029195$

H, -1.355735, -0.187615, 4.429966

$\mathrm{H}, 0.14424,-1.140592,4.495813$

C, $-2.584786,2.839969,-2.106196$

H, $-2.463327,3.553175,-1.287744$

$\mathrm{H},-2.500815,3.379852,-3.05242$

C, $2.704788,-2.324834,1.293425$

H, 2.078588, -1.790809, 2.012502

H, 2.951434, -3.303886, 1.711677

C, $-1.987548,-3.694574,-2.056462$

$\mathrm{H},-1.931356,-4.066987,-3.082134$

H, $-1.595942,-4.462002,-1.385378$

H, $-3.038597,-3.516773,-1.814978$

H, -1.562167, -1.642861, -2.600266

H, 3.635941, $-1.769409,1.158658$

$\mathrm{H},-3.585801,2.404971,-2.048582$

H, 2.420554, $-0.683353,-0.764663$

$\mathrm{H},-0.523485,2.1847,-2.068778$

H, -1.630788, 1.044171, -2.852266 
$\mathrm{H}, 1.054631,-3.052523,0.095367$

H, $-2.467562,0.570317,-0.720506$

H, $-0.238963,-1.497081,2.111614$

O, 3.566751, $0.681645,-0.954821$

C, 4.801115, 0.666437, -0.204823

$\mathrm{H}, 5.239519,1.665053,-0.235263$

$\mathrm{H}, 4.509388,0.445623,0.82052$

C, 5.757545, $-0.372934,-0.747174$

$\mathrm{H}, 6.02828,-0.143808,-1.781074$

H, 6.672619, $-0.384438,-0.15007$

H, 5.309277, -1.368674, -0.714645

H, 3.752462, 0.919374, - 1.876732

O, $-3.697826,-0.644453,-0.260822$

H, $-4.438971,-0.739242,-0.87905$

C, $-4.209401,-0.328798,1.055155$

H, -3.349364, -0.398594, 1.719132

H, $-4.933209,-1.093329,1.339957$

C, $-4.820562,1.055707,1.093066$

H, -5.660004, 1.12844, 0.39704

$\mathrm{H},-5.191251,1.267204,2.099151$

$\mathrm{H},-4.079123,1.814243,0.831582$

\section{$\left[\mathrm{Zn}(\mathrm{EtOH})_{6}\right]^{2+}(\mathrm{EtOH})$}

Zn, 0.430497, 0.054959, 0.184173

O, $-1.228728,1.182228,-0.560743$

$\mathrm{O},-1.086894,-1.217248,0.955814$

$\mathrm{O}, 0.453397,0.946787,2.129154$

$\mathrm{O}, 1.992688,-1.270454,0.896151$

O, 1.907251, 1.566158, - 0.220614

O, $0.743523,-0.874277,-1.726519$

C, $0.377349,-2.273283,-1.842448$

H, $-0.69917,-2.369759,-1.694108$

H, 0.891341, -2.771966, -1.023399

C, $-0.828374,-2.360162,1.799326$

H, $-0.113718,-2.018558,2.546795$

$\mathrm{H},-0.360559,-3.146215,1.204278$

C, $-1.00045,2.196528,-1.565288$

$\mathrm{H},-0.659751,1.718783,-2.48711$

$\mathrm{H},-0.196068,2.816782,-1.176016$

C, $-0.165331,2.248865,2.296065$

H, $-1.248541,2.124513,2.285392$

$\mathrm{H}, 0.131631,2.822281,1.420892$

C, 2.586451, 1.720191, - 1.491541

H, 1.79988, 1.715667, -2.24247

H, 3.233349, 0.857998, -1.656219

C, 3.269339, -1.417489, 0.229448

H, 3.833363, $-0.489447,0.339809$

$\mathrm{H}, 3.031928,-1.563204,-0.821376$
C, $-2.251772,3.013613,-1.809002$

H, $-2.599696,3.471345,-0.880366$

$\mathrm{H},-2.034713,3.808836,-2.526307$

$\mathrm{H},-3.050805,2.392831,-2.22048$

C, 4.030914, $-2.597283,0.793829$

H, 3.460117, $-3.520166,0.671292$

$\mathrm{H}, 4.983525,-2.705029,0.26965$

H, 4.241694, -2.450396, 1.856202

C, $0.310343,2.90318,3.57392$

$\mathrm{H},-0.155164,3.886196,3.679148$

$\mathrm{H}, 0.037213,2.30183,4.444476$

$\mathrm{H}, 1.394461,3.033331,3.559944$

C, $0.803865,-2.828798,-3.18286$

$\mathrm{H}, 0.545236,-3.888871,-3.237865$

$\mathrm{H}, 0.294304,-2.311046,-3.999401$

$\mathrm{H}, 1.88252,-2.726804,-3.318563$

C, 3.368431, 3.014843, -1.524811

$\mathrm{H}, 3.854068,3.124958,-2.497369$

$\mathrm{H}, 2.708368,3.870166,-1.36639$

H, 4.143977, 3.020069, -0.754726

C, $-2.108588,-2.844555,2.44612$

H, $-1.893421,-3.699108,3.092136$

$\mathrm{H},-2.559314,-2.05609,3.052324$

H, $-2.829118,-3.163842,1.688487$

$\mathrm{H},-1.937847,0.566595,-0.851938$

$\mathrm{H}, 0.294061,-0.377508,-2.428402$

$\mathrm{H}, 2.150863,-1.10941,1.840324$

$\mathrm{H}, 0.068513,0.336871,2.778518$

$\mathrm{H},-1.744986,-1.44025,0.266126$

$\mathrm{H}, 2.567246,1.536676,0.491119$

O, $-3.055723,-0.875476,-0.934445$

C, $-4.277291,-0.754219,-0.169055$

H, $-4.816309,-1.701292,-0.223328$

H, -3.955446, $-0.594058,0.859183$

C, $-5.129839,0.397537,-0.657418$

$\mathrm{H},-6.055955,0.439401,-0.078349$

H, $-4.604581,1.34677,-0.535599$

H, -5.391457, 0.268584, -1.710786

$\mathrm{H},-3.270363,-1.181785,-1.828824$

$\left[\mathrm{Zn}(\mathrm{EtOH})_{6}\right]^{2+}(\mathrm{EtOH})_{2}$

Zn, 0.097686, 0.114313, -0.044701

O, -1.226428, 1.497361, 0.898702

O, -1.359559, -1.392672, 0.395016

$\mathrm{O}, 1.322361,-0.158589,1.733692$

O, 1.393628, -1.494466, -0.667385

$\mathrm{O}, 1.528339,1.661387,-0.396116$

O, $-0.791301,0.133526,-2.006595$ 
C, $-2.222948,0.258539,-2.184998$

$\mathrm{H},-2.533303,1.2554,-1.867153$

H, $-2.658954,-0.481856,-1.519568$

C, $-1.030411,-2.45889,1.312366$

H, $-0.924458,-2.044542,2.318089$

$\mathrm{H},-0.065353,-2.842052,0.986314$

C, $-1.14792,2.915052,1.143379$

$\mathrm{H},-0.118032,3.198011,0.938155$

$\mathrm{H},-1.354251,3.092488,2.200766$

C, 0.981844, 0.564317, 2.938344

$\mathrm{H},-0.043063,0.318501,3.222783$

$\mathrm{H}, 1.02468,1.615656,2.661258$

C, $1.371641,2.577533,-1.500485$

$\mathrm{H}, 0.325164,2.880857,-1.493227$

$\mathrm{H}, 1.580772,2.047836,-2.433632$

C, 1.06834, -2.390991, -1.749469

$\mathrm{H}, 1.19944,-1.868398,-2.698854$

$\mathrm{H}, 0.013008,-2.629115,-1.629697$

C, $-2.11947,3.674387,0.263532$

$\mathrm{H},-3.146593,3.352415,0.456444$

$\mathrm{H},-2.053269,4.744601,0.475133$

H, -1.893648, 3.515949, -0.793142

C, 1.923258, -3.639018, -1.685397

$\mathrm{H}, 1.781743,-4.156101,-0.733524$

H, 1.646168, -4.319066, -2.494787

H, 2.982112, -3.391461, -1.795995

C, 1.953435, 0.247683, 4.054545

$\mathrm{H}, 1.680286,0.812808,4.94895$

$\mathrm{H}, 1.925512,-0.816523,4.303557$

H, 2.973266, 0.517125, 3.772172

C, $-2.607447,-0.009836,-3.62379$

$\mathrm{H},-3.692258,0.063679,-3.733102$

H, $-2.147804,0.721228,-4.293657$

H, -2.295299, -1.011748, -3.925844

C, 2.289426, 3.770822, -1.335336

H, 2.152121, 4.465137, - 2.167685

H, 2.071068, 4.297613, -0.403726

H, 3.335093, 3.453405, -1.322228

C, $-2.088181,-3.542446,1.277277$

H, $-1.799782,-4.361596,1.940447$

H, $-3.054034,-3.157425,1.613066$

H, $-2.199521,-3.938323,0.265276$

H, $-2.120983,1.163144,1.115643$

H, $-0.350163,0.77596,-2.584871$

H, 2.29108, $-1.119865,-0.800027$

H, 1.364226, -1.107683, 1.931489

H, -2.210025, $-0.988776,0.677461$

$\mathrm{H}, 2.390839,1.19921,-0.491535$

O, 3.720908, 0.026957, -0.827811

$\mathrm{H}, 4.174629,0.149224,-1.676092$

O, -3.554474, 0.043055, 1.302243
H, -3.784365, -0.272066, 2.189828

C, $4.705308,-0.096347,0.224239$

H, 5.323273, 0.802991, 0.23014

H, 4.130698, $-0.131006,1.147822$

C, 5.54538, -1.343987, 0.051646

H, 6.091731, $-1.315605,-0.89448$

H, 6.273761, $-1.413096,0.863333$

$\mathrm{H}, 4.918632,-2.238342,0.071402$

C, $-4.755695,0.13343,0.502531$

$\mathrm{H},-4.484509,0.7333,-0.364149$

$\mathrm{H},-5.51355,0.672099,1.072774$

C, $-5.246013,-1.237876,0.088311$

$\mathrm{H},-5.515284,-1.835522,0.962563$

H, -6.133907, -1.135691, - 0.540951

H, -4.478541, -1.768851, - 0.48006

$\left[\mathrm{Zn}(\mathrm{OAc})(\mathrm{EtOH})_{2}\right]^{+}$

$\mathrm{Zn}, 0.026058,-0.219021,0.021722$

O, - $0.605502,1.5534,0.940779$

$\mathrm{O}, 0.495138,1.657901,-0.953983$

$\mathrm{O},-1.606254,-1.331532,-0.401637$

O, 1.738826, -1.238827, 0.387811

C, $-0.111694,2.245253,-0.007956$

C, $-0.279161,3.73511,-0.001809$

H, 0.389191, 4.205175, - 0.719754

$\mathrm{H},-1.313975,3.963423,-0.270256$

H, $-0.099947,4.124926,1.000253$

C, $2.98259,-0.640445,-0.08578$

C, $4.100335,-1.655649,-0.04248$

H, 3.864493, - 2.516538, -0.670728

H, 5.020979, $-1.197344,-0.41113$

H, 4.276122, $-1.997983,0.980076$

$\mathrm{H}, 2.770042,-0.320781,-1.10303$

$\mathrm{H}, 3.198112,0.234459,0.527559$

H, 1.833074, -1.484588, 1.322618

C, $-2.886784,-0.86798,0.112874$

C, $-3.930296,-1.951763,-0.025263$

$\mathrm{H},-4.875705,-1.598505,0.393066$

H, -4.095576, -2.202395, -1.075908

$\mathrm{H},-3.625741,-2.851881,0.511886$

$\mathrm{H},-2.697988,-0.62465,1.155661$

$\mathrm{H},-3.167667,0.039528,-0.421448$

H, -1.68476, - $1.516905,-1.351806$

$\left[\mathrm{Zn}(\mathrm{OAc})(\mathrm{EtOH})_{2}\right]^{+}(\mathrm{EtOH})$ 
Zn, 0.691655, 0.034431, 0.196146

$\mathrm{O}, 2.599933,-0.843201,0.268643$

$\mathrm{O}, 2.21878,0.890325,-1.016115$

$\mathrm{O},-0.533536,1.187335,1.312633$

O, $-0.539333,-1.461604,-0.52709$

C, 3.021616, 0.007671, -0.576281

C, 4.444296, $-0.010815,-1.046533$

$\mathrm{H}, 4.466133,0.003597,-2.137063$

$\mathrm{H}, 4.940132,0.89531,-0.690903$

$\mathrm{H}, 4.968443,-0.886255,-0.670221$

C, $-0.325127,-2.77172,0.065025$

C, $-0.357583,-2.694214,1.57642$

$\mathrm{H},-1.3347,-2.353358,1.927959$

$\mathrm{H},-0.160635,-3.678938,2.006807$

$\mathrm{H}, 0.407992,-2.000629,1.936581$

H, -1.095276, -3.438619, -0.323104

$\mathrm{H}, 0.644982,-3.101507,-0.300596$

$\mathrm{H},-1.437985,-1.145118,-0.279234$

C, $-0.353494,2.625923,1.254144$

C, $-0.735755,3.180009,-0.100041$

$\mathrm{H},-0.55182,4.25699,-0.118534$

H, $-0.143393,2.716487,-0.893076$

H, $-1.796115,3.011842,-0.302172$

$\mathrm{H},-0.955565,3.063907,2.050163$

$\mathrm{H}, 0.698808,2.792356,1.474987$

$\mathrm{H},-1.452776,0.93515,1.062047$

O, $-2.761504,-0.063663,0.308397$

C, $-3.46509,0.467195,-0.838865$

$\mathrm{H},-4.102771,1.289287,-0.510759$

H, $-2.687831,0.863525,-1.490582$

C, $-4.265363,-0.613216,-1.533572$

H, -5.023737, -1.025015, -0.862856

$\mathrm{H},-4.772786,-0.196251,-2.406718$

H, $-3.612535,-1.423476,-1.86655$

H, $-3.403935,-0.430741,0.935902$

\section{$\left[\mathrm{Zn}(\mathrm{OAc})(\mathrm{EtOH})_{2}\right]^{+}(\mathrm{EtOH})_{2}$}

Zn, - $1.447998,-0.125213,0.081476$

$\mathrm{O},-2.894806,-1.683613,-0.282982$

$\mathrm{O},-3.454463,0.278716,0.515584$

O, $-0.566929,1.017457,-1.370407$

O, 0.137976, -0.689822, 1.226126

C, $-3.790958,-0.890622,0.136684$

C, $-5.232652,-1.299339,0.154803$

$\mathrm{H},-5.73771,-0.868433,1.018322$

H, $-5.707396,-0.906511,-0.748521$

H, $-5.325488,-2.383533,0.156226$

C, $0.592238,-2.05641,1.03188$
C, $0.91486,-2.329058,-0.421857$

H, 1.689769, -1.649991, - 0.785317

$\mathrm{H}, 1.275628,-3.354333,-0.535974$

$\mathrm{H}, 0.023231,-2.20721,-1.043931$

$\mathrm{H}, 1.462438,-2.2052,1.672069$

H, $-0.218539,-2.687919,1.389461$

$\mathrm{H}, 0.849603,-0.069803,0.924833$

C, $-1.265248,2.23023,-1.756475$

C, $-1.337892,3.209164,-0.605143$

$\mathrm{H},-1.916983,4.087538,-0.899716$

$\mathrm{H},-1.825148,2.749056,0.259491$

H, $-0.338274,3.540797,-0.314153$

$\mathrm{H},-0.739422,2.655062,-2.611622$

H, -2.254571, 1.906816, -2.074108

$\mathrm{H}, 0.311448,1.248272,-0.98511$

$\mathrm{O}, 1.712768,1.231563,0.15293$

C, 2.002379, 2.201253, 1.18042

$\mathrm{H}, 2.425963,3.094155,0.716763$

$\mathrm{H}, 1.039841,2.461653,1.619681$

C, 2.938772, 1.626281, 2.224709

$\mathrm{H}, 3.907617,1.379334,1.784234$

$\mathrm{H}, 3.102479,2.356729,3.020859$

$\mathrm{H}, 2.512311,0.722537,2.668114$

$\mathrm{H}, 2.54745,0.93676,-0.281582$

O, 3.862057, 0.097337, -1.06829

$\mathrm{H}, 4.511613,0.568734,-1.610424$

C, 4.542952, $-0.863762,-0.240426$

$\mathrm{H}, 5.344464,-0.365262,0.308414$

H, 3.798186, -1.208931, 0.477049

C, 5.080895, - $2.016557,-1.063247$

H, 5.572331, $-2.74364,-0.411858$

H, 4.270865, - 2.519157, -1.596681

H, 5.813332, -1.661324, -1.792581

$\left[\mathrm{Zn}(\mathrm{OAc})_{2}\right]$

$\mathrm{Zn},-0.000001,0,-0.0037$

O, 1.799527, 0.913437, 0.615009

O, $-1.802727,0.893171,-0.623718$

C, 2.445991, 0.013331, -0.005944

C, $-2.44599,-0.013332,-0.005935$

O, $1.802725,-0.893172,-0.623723$

$\mathrm{O},-1.799523,-0.913437,0.615014$

C, $3.94403,-0.005849,0.012584$

C, $-3.94403,0.005849,0.012583$

$\mathrm{H}, 4.26377,-0.739404,0.757609$

$\mathrm{H}, 4.327571,-0.31968,-0.957552$

$\mathrm{H}, 4.340604,0.969914,0.285278$

H, -4.263782, 0.739623, 0.757385 
H, -4.327554, 0.319407, -0.957649

H, $-4.340607,-0.969839,0.285539$

$\left[\mathrm{Zn}(\mathrm{OAc})_{2}(\mathrm{EtOH})\right]$

Zn, 0.329854, -0.253127, -0.130427

$\mathrm{O},-0.741824,1.443597,-0.532787$

$\mathrm{O},-2.787612,-0.206666,-0.052522$

$\mathrm{O}, 2.088169,-0.138064,1.101804$

$\mathrm{O}, 2.220864,-0.585589,-1.042284$

$\mathrm{O},-1.075338,-1.652275,-0.072664$

C, 2.793442, $-0.387616,0.075276$

C, $-2.322894,-1.366414,0.035213$

C, $-0.577707,2.567719,0.366434$

$\mathrm{H}, 0.448551,2.904718,0.231454$

H, $-0.70316,2.219526,1.393547$

C, $-1.571422,3.657651,0.028491$

H, -1.426356, 4.510896, 0.69548

$\mathrm{H},-1.4376,3.993455,-1.001907$

H, $-2.595373,3.296252,0.151778$

$\mathrm{H},-1.629815,1.01869,-0.383172$

C, $4.28743,-0.474735,0.173857$

$\mathrm{H}, 4.648079,0.038499,1.062966$

$\mathrm{H}, 4.564001,-1.530612,0.236517$

H, 4.745376, $-0.057196,-0.722348$

C, $-3.240738,-2.531783,0.303047$

$\mathrm{H},-3.028916,-3.339567,-0.398781$

H, $-3.039042,-2.905651,1.310242$

H, $-4.284102,-2.232145,0.230767$ 